\title{
Dirac and Plateau Billiards in Domains with Corners.
}

\author{
Misha Gromov
}

November 13, 2018

\section{Contents}

1 Setting the Stage. 2

1.1 Surgery at the Corners. . . . . . . . . . . . . . . . . . . 3

1.2 Mean Convexity and Dihedral Angles. . . . . . . . . . . . . . 4

1.3 Mean Convexity under Surgery. . . . . . . . . . . . . . . . 6

1.4 Mean Curvature Stability and Semistability Problems. . . . . . . 6

1.5 Dihedral Extremality and Plateau-hedra. . . . . . . . . . . . 8 8

1.6 Euclidean Dihedral Extremality Problem. . . . . . . . . . . . 9

1.7 On Acute Polyhedra. . . . . . . . . . . . . . . . . . 12

$1.8 C^{0}$-Limits of Metrics with $s c a l \geq \kappa \ldots \ldots \ldots \ldots \ldots \ldots$

1.9 Rigidity Problems around $s c a l \geq 0 \ldots \ldots \ldots \ldots \ldots$

1.10 Singular Spaces with scal $\geq 0$ and Related Problems. . . . . . 16

2 Reflection Domains. 18

2.1 Gluing around Edges and Dihedral Extremality Theorem. . . . . 19

2.2 Dihedral Rigidity Conjecture. . . . . . . . . . . . . . . . 24

2.3 Billiards, Pure Edges and Ramified Coverings. . . . . . . . . 28

$3 \mu$-Area and $\mu$-Bubbles. 29

3.1 Poly-Bubble-Hedra. . . . . . . . . . . . . . . . . . . . 31

3.2 Multi-bubble Description and Construction of PB-hedra. . . . . . 32

4 Plateau Traps, $\phi$-Convexity, Qiasiregularity and Regularization.

4.1 Directed Homology. . . . . . . . . . . . . . . . . . . . 34

4.2 Traps and Walls . . . . . . . . . . . . . . . . . . . 34

4.3 Smoothing and Doubling. . . . . . . . . . . . . . . . 36

$4.4 \quad \Delta$-Stable Mean Convexity. . . . . . . . . . . . . . . 37

4.5 $C^{0}$-Stability of the Mean Curvature and Reifenberg Flatness. . 40

4.6 Reifenberg Flatness and Filling Profile. . . . . . . . . . . . 42

4.7 Sharp Filling under Reifenberg's Controle. . . . . . . . . . . . . 45

4.8 Reifenberg and Höder at the Corners. . . . . . . . . . . . . . 46

4.9 Proof of the $C^{0}$-Limit Theorem. . . . . . . . . . . . 48 
5 Conjectures and Problems.

5.1 On Topography of Plateau Wells. . . . . . . . . . . . . . 50

5.2 Webs and Honeycombs. . . . . . . . . . . . . . . 51

5.3 Nested Cubes and Small Diameter Conjecture. . . . . . . . . 52

5.4 Gauss Bonnet Prism Inequalities and the Extremal Model Problem. 55

$5.5 \quad$ Spaces or Objects? . . . . . . . . . . . . . . . . . . . 61

6 Bibliography. 62

\begin{abstract}
Groping our way toward a theory of singular spaces with positive scalar curvatures we look at the Dirac operator and a generalized Plateau problem in Riemannian manifolds with corners. Using these, we prove that

the set of $C^{2}$-smooth Riemannian metrics $g$ on a smooth manifold $X$, such that $\operatorname{scal}_{g}(x) \geq \kappa(x)$, is closed under $C^{0}$-limits of Riemannian metrics for all continuous functions $\kappa$ on $X$.

Apart from that our progress is limited but we formulate many conjectures. All along, we emphasize geometry, rather than topology of manifolds with their scalar curvatures bounded from below.
\end{abstract}

\title{
1 Setting the Stage.
}

A closed subset $P=P^{n}$ in a smooth $n$-manifold $X$ is called a cornered or curvefaced polyhedral $n$-domain of depth $d=0,1, \ldots, n$ if the boundary of $\partial P$ of $P$ is decomposed into the union of a countable locally finite (e.g. finite) family of (possibly disconnected) $(n-1)$-faces $Q_{i}=Q_{i}^{n-1}$ with a distinguished set of adjacent pairs of faces $\left(Q_{i}, Q_{j}\right)$, such that

- every face $Q_{i}$ is contained in a smooth hypersurface $Y_{i}=Y_{i}^{n-1} \subset X$, where $Y_{i}$ is transversal to $Y_{i}$ for all adjacent pairs of $(n-1)$-faces;

-• the boundary $\partial Q_{i}$ of each $Q_{i} \subset Y_{i}$ equals the union of the intersections $Q_{i} \cap Q_{j}$ for all faces $Q_{j}$ that are adjacent to a given $Q_{i}$, where the corresponding decompositions $\partial Q_{i}=\cup_{j} Q_{i} \cap Q_{j}$ give polyhedral $(n-1)$-domain structures of depth $d-1$ to all $Q_{i}$.

This defines the notion of a polyhedral domain structure by induction on $d$, where polyhedral domains of depth zero are non-empty closed subsets $P \subset X$ with empty boundaries, i.e. just smooth manifolds with no extra structures and domains of depth one are those bounded by smooth hypersurfaces.

A polyhedral domain $P$ is called cosimplicial if the intersections of all $k$ tuples of mutually non-equal $(n-1)$-faces satisfy

$$
\operatorname{dim}\left(Q_{i_{1}} \cap Q_{i_{2}} \cap \ldots \cap Q_{i_{k}}\right) \leq n-k+1 .
$$

Polyhedra, Edges, Corners. Our attention will be focused on the boundaries of cornered domains that are unions of faces, $\cup_{i} Q_{i}$. We call these boundaries curve-faced polyhedra or polyhedral hypersurfaces and may occasionally denote them $P$ rather than $\partial P$.

The codimension two faces of $P$ that are (n-2)-dimensional intersections of faces $Q_{i}$ are called edges of $P$ and the higher codimension faces are called corners. Thus, corners appear starting from $d=3$, such as the ordinary corners of a cube in the 3 -space. 
A cornered $n$-manifold structure in a $P$ is defined by an extension of $P$ to a smooth manifold $X \supset P$ where $P$ makes a polyhedral domain. The tangent bundle $T(P)$ equals, by definition, the tangent bundle of $X$ restricted to $P$.

A Riemannian manifold with corners is a cornered $n$-manifold $P$ with a Riemannian metric $g=g_{P}$ on $T(P)$.

Cartesian products of cornered manifolds, $P=P_{1} \times P_{2} \times \ldots \times P_{m}$, come with natural (product) corner structures where $\operatorname{dim}(P)=\operatorname{dim}\left(P_{1}\right)+\operatorname{dim}\left(P_{2}\right)+\ldots+$ $\operatorname{dim}\left(P_{m}\right)$ and $\operatorname{depth}(P)=\operatorname{depth}\left(P_{1}\right)+\operatorname{depth}\left(P_{2}\right)+\ldots+\operatorname{depth}\left(P_{m}\right)$. If $P_{i}$ are Riemannian, then the product carries the natural Riemannian Cartesian product structure as well.

The simplest instance of this is the unit Euclidean n-cube, sometimes denoted $\square$ or $\square^{n}$ (instead of more logical $\square^{n / 2}$ ) that is the Cartesian $n$-th power $[0,1]^{n}$ of the segment $[0,1]$.

A cornered Riemannian manifold $P$ is called preconvex, if the dihedral angles, denoted $\angle\left[Q_{i} \pitchfork Q_{j}\right]$, that are continuous functions on the edges of $P$, that are the $(n-2)$-faces $Q_{i} \cap Q_{j}$, are $<\pi$ for all pairs of adjacent $(n-1)$-faces $Q_{i}$ and $Q_{j}$.

Observe that preconvexity, does not depend on the metric and that intersections of finitely many domains with smooth mutually transversal boundaries in smooth manifolds $X$ are preconvex.

\subsection{Surgery at the Corners.}

The simplest instance of surgery is the following operation of

Adding 1-handle at the corners. Let $P \subset X=X^{n}$ be a polyhedral domain and let $A^{1} \subset X$ be a smooth curve, (future axis of the surgery) that joins two vertices $b_{i}$ and $b_{2}$ vertices (i.e. codimension $n$ faces) in $P$ and that does not meet $P$ apart from these points.

Suppose there exists a face preserving diffeomorphism $D$ between small neighbourhoods $U_{1}$ and $U_{2}$ of $b_{1}$ and $b_{2}$ in $P$. (If $X$ is a Riemannian manifold one thinks of these neighbourhoods as intersections of the Riemannian $\varepsilon$-balls $B_{b_{1}}(\varepsilon)$ and $B_{b_{2}}(\varepsilon)$ with $P$.)

Notice that such a diffeomorphism does exist if $P$ is cosimple at these vertices.

The boundaries $\partial U_{1}$ and $\partial U_{2}$, that are identified by $D$, carry natural structure of cornered manifold of dimension $n-1$ call it $Q^{n-1}$. Then the curve $A^{1}$ can be thickened in $X$ to $A^{1} \times Q^{n-1}$ and glued to $P \backslash\left(U_{1} \cup U_{2}\right)$ at its two (thickened) ends, where the resulting domain, call it $P^{\prime}$ carries a natural cornered structure. Notice that $P^{\prime}$ has the same vertices as $P$, except that $b_{1}$ and $b_{2}$ are not there anymore.

Example: Doubling. Let $P$ consist of two disjoint diffeomorphic polyhedral domains. Then upon performing the above surgery over all pairs of corresponding vertices one obtains a new domain $P^{\prime \prime}$ of depth $n-1$, i.e. with no vertices at all.

Remark. The above works as stated only if $\operatorname{dim}(X \geq 3)$ where one may assume the curves joining the vertices do not have to intersect, but if $n=2$ then $P^{\prime}$ can be a priori immersed rather than imbedded into $X$, that is, however, rather immaterial for our purposes. 
Now, in general let $B^{m-1} \subset P$ be an $(m-1)$-dimensional face in $P$ that contains no lower dimensional faces. For instance, this may be a a closed 1-face in the above $P^{\prime \prime}$.

Let $A^{m} \subset X$ be an $m$-submanifold with $\partial A^{m}=B^{m-1}$ that meets $P$ only at $B^{m-1} \subset P$. Let the normal corner structure of $B^{m-1} \subset P$ is represented by a flat fibration $U \rightarrow B^{m-1}$ with the fiber $C^{n-m+1}$, that is the cone over a cornered manifold $Q^{n-m}$

(Such a $C^{n-m}$ automatically exists if $B^{m-1}$ is connected; if $B^{m-1}$ is simply connected then, moreover, its small neighbourhood in $P$ splits as $B^{m-1} \times$ $C^{n-m+1}$.)

Let $W^{n} \rightarrow A^{m}$ be an extension of the flat fibration $U$ from $B^{m-1}$ to $A^{m} \supset$ $B^{m-1}$. Then one can remove $U$ from $P$ and attach $W$ instead.

Example: Multi-doubling. Take two disjoint copies of the above doubled $P^{\prime \prime}$ and join the unions of their curves of edges by a (possibly disconnected) cylinder $A^{2}$. Then the result of the corresponding surgery, say $\left(P^{\prime \prime}\right)^{\prime \prime}$ will have depth $(n-2)$. If we repeat this $n$ times we arrive at a manifold $P^{*}$ of depth zero that is a slowed manifold with no corners of any kind.

Face Suppression. If one double $P^{*}$ along the boundary, one obtains a closed manifold with "pure edge singularities" (as in section 2.3).

Remark. The above kind of surgery applies to all spaces with normally conical singularities. Thus, for example, every pseudomanifold $P$ can be turned to a manifold $P^{*}$ that is "kind of cobordant" to $P$.

\subsection{Mean Convexity and Dihedral Angles.}

Call a preconvex Riemannian manifold $P$ with corners mean curvature convex, and write mn.curv $(\partial P) \geq 0$ if all $(n-1)$-faces $Q_{i}=Q_{i}^{n-1}$ have (non-strictly) positive mean curvatures, i.e. the variations of the $(n-1)$-volumes of the faces $Q_{i}$ are non-positive under infinitesimal inward deformations of $Q_{i}$.

Remarks and Examples. (a) The simplest instance of a mean convex domain in $X$ is a full dimensional submanifold $U \subset X$ with a smooth boundary that has positive mean curvature. Such domains are abundant in $X$. For instance every piecewise smooth subset $Z \subset X$ of codimension $\geq 2$ in $X$ admits an arbitrarily small smooth mean convex neighbourhood.

Furthermore, if $U$ is smooth mean convex, then the union $U \cup Z$ admits a smooth mean convex neighbourhood in $X$.

(b) The intersection of finitely many domains $U_{i} \subset X$ with mutually transversal smooth mean convex (e.g. convex) boundaries $\partial U_{i}$ is an m.c. convex polyhedral domain in $X$ with the faces made of pieces of $\partial U_{i}$.

(c) If $P$ is compact and the faces have strictly positive mean curvatures, then (it is obvious, see 22 for related results) the boundary of a an $\varepsilon$-neighbourhood of $P$, call it $\partial P_{+\varepsilon}$, is $C^{1,1}$, smooth for small $\varepsilon>0$ and has (discontinuous ) positive mean curvature.

It easily follows that a mean convex $\partial P$ can be approximated by $C^{2}$-smooth hypersurfaces with mn.curv $>0$, unless (some connected component of) $\partial P$ consists of a single face with zero mean curvature.

But this is not especially relevant in the present context: we are keen at keeping track of the combinatorial pattern of the corners of $P$ and of the dihedral angles between adjacent $(n-1)$-faces at the corners. 
The combinatorial type/scheme $\mathcal{C} \mathcal{T}=\mathcal{C} \mathcal{T}(P)$ of a manifold $P$ with corners refers to the intersection and the adjacency patterns between its codimension one faces $Q_{i}$.

Observe, that the combinatorial type is stable under Cartesian products of $P$ by connected manifolds (without boundaries) with their tautological corner structures of depth zero.

Combinatorial equivalence. We often call two domains combinatorially equivalent if they are of the same combinatorial type. Notice that such domains do not even have to be of the same dimension.

Cubical Domains $P$ of Depth $d$ in $n$-Manifold $X$. Such a $d$-cubical domain $P$ has $2 d$ faces $Q$ of codimension one, where every face $Q$ has a unique opposite face, call it $-Q$, which does not intersect $Q$. Every cubical $P$ admits a continuous map onto the unit $d$-cube, $(P, \partial P) \rightarrow\left(\square^{d}, \partial \square^{d}\right)$, with the faces of $P$ being the pullbacks of the faces of $\square^{d}$, where, such a map is uniquely, up to homotopy in the class of faces-to-faces maps, is determined of by to which faces of the cube the faces of $P$ go.

An $n$-cubical $P$ is called essential if the map $(P, \partial P) \rightarrow\left(\square^{n}, \partial \square^{n}\right)$ has nonzero degree, where the degree is understood $\bmod 2$ if $P$ is not oriented.

Combinatorial Mean Curvature Convexity Problem. When does a Riemannian manifold $X$, e.g. $X=\mathbb{R}^{n}$, contain a mean curvature convex polyhedron $P=P_{\leq \alpha_{i j}}$ of a given combinatorial type where all its dihedral angles are bounded by $<_{i j}(P) \leq \alpha_{i j}$ for given constants $\alpha_{i j}$ ?

This is unknown even for $X=\mathbb{R}^{3}$, where the answer is available only for "normal" mean curvature convex domains $P \subset \mathbb{R}^{3}$ that are combinatorially equivalent to prisms, where "normal" means that the dihedral angles at the top and the bottom of these "prisms" are equal $\frac{\pi}{2}$ and where a simple argument (see section 5.4) shows that

the dihedral angles $\alpha_{i}$ between the side faces of these $P$ satisfy

$$
\sum_{i}\left(\pi-\alpha_{i}\right) \leq 2 \pi
$$

with the equality only for convex prisms $P \subset \mathbb{R}^{3}$ with flat faces.

The inequality (*) remans true for "prisms" $P$ with Riemannian metrics with non-negative scalar curvatures (see section 5.4) and it suggests a possibility of defining general spaces $X$ with non-negative scalar curvatures, at least in dimension 3 , via this $(*)$. But it is unclear what kind of singularities one may admit in such a definition.

The simplest case where we do not know the answer is that of continuous Riemannian metrics on smooth 3 -manifolds $X$.

Here, the mean curvature convexity of hypersurfaces may be defined as in section 4.4 by the existence of "many" localized inward deformations of faces that are area decreasing. (This defines, as stated, strictly positive mean curvature.)

Alternatively (that is not, in general, equivalent) one may requite all small localized outward deformations of the faces to be area increasing.

Or, one may require both: increase of area for all outward deformation and decrees for "many" inward deformations. 
Test Question. Suppose that all sufficiently small prisms $P=P_{x} \subset X$ that contain $x \in X$ satisfy $(*)$ for all $x \in X$. Do then all $P \subset X$ satisfy $(*)$ ?

(This question is motivated by a similar localization property of the comparison inequalities for geodesic triangles in Alexandrov's spaces with positive curvatures.)

\subsection{Mean Convexity under Surgery.}

Let $P$ be a strictly mean curvature convex polyhedral domain in a Riemannian $n$-manifold $X$ with all dihedral angles of $P$ bounded by $\angle_{i j}(P)<\alpha_{i j}$. Let $P^{\prime}$ be obtained by a surgery along some union $B^{m-1} \subset P$ of $(m-1)$-faces that themselves have no corners (see section 1.1).

Observe, that in general when $2 m \geq n$, such a $P^{\prime}$ is immersed rather than embedded into $X$.

If $n-m \geq 2$, then $P^{\prime}$, with its combinatorial structure coming along with the surgery, can be arranged (i.e. immersed) in $X$ with strictly positive mean curvature of all its faces and with all dihedral angles satisfying $<_{i j}\left(P^{\prime}\right)<\alpha_{i j}$

This is immediate with (a simplified version of) the argument from [24] for a similar behavior of positive scalar curvature under surgery.

Corollary. The non-strict version of the above combinatorial mean curvature convexity problem, that is of the existence of $P=P_{<\alpha_{i j}}$, is invariant under the codimension $\geq 2$-surgery.

Example. Every mean curvature strictly convex $P=P_{<\alpha_{i j}}$ can be transformed by multi-doubling to a $P^{\prime \prime} \cdots$ without corners, i.e. of depth 2 , where

mean.curv $(P)>0 \&<_{i j}(P)<\alpha_{i j} \Rightarrow$ mean.curv $\left(P^{\prime \prime} \cdots\right)>0 \&<_{i j}\left(P^{\prime \prime} \cdots\right)<\alpha_{i j}$.

Question. Does the reverse implication also hold true?

Namely, does the existence of a position (i.e. of an immersed) $P^{\prime \prime} \cdots$ in $X$ with strictly positive mean curvatures of the faces and all dihedral angles strictly bounded by given $\alpha_{i j}$ imply the existence of such a position for $P$ in $X$ ?

\subsection{Mean Curvature Stability and Semistability Prob- lems.}

Conjecturally, the existence of the above $P_{<\alpha_{i j}}$, is stable under smooth perturbations of the Riemannian metric $g$ in $X \supset P$ that are $\varepsilon$-small in the $C^{0}$-topology.

More generally, let $P \subset X$ be a compact strictly preconvex (i. e. all $\alpha_{i j}<\pi$ ) polyhedral domain in a smooth (meaning $C^{\infty}$ ) manifold $X$ with a $C^{2}$-smooth Riemannian metric $g$. Let $X^{\prime}=X_{\varepsilon}=\left(X_{\varepsilon}, g_{\varepsilon}\right)$ be another Riemannian manifold with $C^{2}$-smooth metric $g^{\prime}=g_{\varepsilon}$ and let $f_{\varepsilon}: X^{\prime} \rightarrow X$ be a continuous map.

An essential example is where $\operatorname{dim}\left(X^{\prime}\right)=\operatorname{dim}(X)$ and $f_{\varepsilon}$ is a $e^{\varepsilon}$-bi-Lipschitz homeomorphism. Also we allow $\operatorname{dim}\left(X^{\prime}\right)>\operatorname{dim}(X)$ where $f_{\varepsilon}$ is $\varepsilon$-close in some sense to a Riemannian submersion.

We seek conditions on $X^{\prime}$ and on $f_{\varepsilon}$ that would guarantee the existence of another map, say $f_{\varepsilon}^{\prime}: X^{\prime} \rightarrow X$ such that

- $\rightarrow 0 f_{\varepsilon}^{\prime}$ is close to $f_{\varepsilon}$, where close may mean $\operatorname{dist}_{X}\left(f_{\varepsilon}^{\prime}\left(x^{\prime}\right), f_{\varepsilon}\left(x^{\prime}\right)\right) \leq \delta(\varepsilon) \rightarrow 0$ for $\varepsilon \rightarrow 0$ and all $x^{\prime} \in X^{\prime}$ or that the function $x^{\prime} \mapsto \operatorname{dist}_{X}\left(f_{\varepsilon}^{\prime}\left(x^{\prime}\right), f_{\varepsilon}\left(x^{\prime}\right)\right)$ is small 
in a weaker (e.g some $L_{p}$ ) topology;

- reg the pullback $P^{\prime}=P_{\varepsilon}^{\prime}=\left(f_{\varepsilon}^{\prime}\right)^{-1}(P) \subset X^{\prime}=X_{\varepsilon}$ is a smooth polyhedral domain with the faces being the $f_{\varepsilon}^{\prime}$-pullbacks of those of $P$.

In order to formulate the stability and the semistability conditions, we agree that the mean curvature of a polyhedral domain at an "edge point" $x$ i.e. at a point where exactly two faces meet signifies

$\pi$ minus the dihedral angle $<_{i j}$ between these faces at $x$.

Now the stability and the semistability conditions read:

•stbl

$$
\left|m n . \operatorname{curv}_{x^{\prime}}\left(P_{\varepsilon}^{\prime}\right)-m n . \operatorname{curv}_{x}(P)\right| \leq \kappa(\varepsilon) \rightarrow 0 \text { for } \varepsilon \rightarrow 0,
$$

for all $x^{\prime}$ at the faces and at the edges of $P_{\varepsilon}^{\prime}$ and $x=f_{\varepsilon}^{\prime}\left(x^{\prime}\right)$;

- semistbl

$$
m n . \operatorname{curv}_{x^{\prime}}\left(P_{\varepsilon}^{\prime}\right)-m n . \operatorname{curv}_{x}(P) \geq-\kappa(\varepsilon) \rightarrow 0 \text { for } \varepsilon \rightarrow 0
$$

for all $x^{\prime}$ at the faces and at the edges of $P_{\varepsilon}^{\prime}$ and $x=f_{\varepsilon}^{\prime}\left(x^{\prime}\right)$.

A particular instance of the latter is where $P$ is strictly mean curvature convex and the same is required of $P_{\varepsilon}^{\prime}$.

On Regularity at the Corners. The conditions $\bullet$ stbl and $\bullet_{\text {semistbl }}$ depend on the faces of $P_{\varepsilon}^{\prime}$ being $C^{2}$-smooth away from the edges and $C^{1}$-smooth at the edges but no regularity at the corners, i.e. at the codimension $\geq 2$ faces is formally needed.

This suggests modified versions of the stability and semistability problems where instead of $\bullet_{\text {reg }}$ we require only $C^{\alpha}$-Hölder smoothness of $P_{\varepsilon}^{\prime}$ at the corners for some $\alpha>0$.

This relaxed regularity condition is easer to satisfy when we construct $P^{\prime}=$ $P_{\varepsilon}^{\prime}$ by means of the geometric measure theory (as we do it in sections 3 and 4).

For instance, let, say a cosimplicial, curve-faced polyhedron $P^{\prime}$ be constructed "face by face" where each $\left(n^{\prime}-1\right)$-face $Q_{i}^{\prime}$ of $P^{\prime}$ for $n^{\prime}=\operatorname{dim}\left(X^{\prime}\right)$ comes as a solution of a Plateau bubble problem (as defined below in section 3) with free boundary contained in the union $Q_{\hat{i}}^{\prime}$ of the remaining faces. (Technically, the solution $Q_{i}^{\prime}$ of this Plateau problem must be kept away from the boundary of $Q_{\hat{i}}^{\prime}$, with the resulting $P^{\prime}$ obtained by chopping away a small neighbourhood of $\partial Q_{\hat{i}}^{\prime} \subset Q_{\hat{i}}^{\prime}$.)

Then, probably(?), one can not guarantee the $C^{2}$-smoothness of $Q_{i}^{\prime}$ at the edge points of the hypersurface $Q_{\hat{i}}$ (although it is likely such $Q_{i}^{\prime}$ are $C^{1}$ at all boundary points where their tangent cones are flat, and possibly, $C^{1, \alpha}$-smooth, even with $\alpha=1$ ) but Hölder can be sometimes obtained. For instance, a Reifenberg flatness argument delivers such Hölder stability for $f_{\varepsilon}$ being a $e^{\varepsilon}$ bi-Lipschitz homeomorphism with a sufficiently small, depending on $X, g$ and $P$, positive $\varepsilon$ (see sections 4.6-4.8).

Insufficient smoothness does not seem to excessively harm essential geometric applications of minimal hypersurfaces as well as of higher codimensional subvarieties.

Moreover, this apparently remans so for more general singular ambient spaces, e.g. for Alexandrov's spaces with curvatures bounded from below. For instance, it seems likely that Almgren's sharp isoperimetric inequality indicated on p. 475 in 20 for smooth manifolds with non-negative sectional curvatures 
extends to singular such spaces $X$ (with the conical spaces $X$ being extremal as pointed out in [20]).

\subsection{Dihedral Extremality and Plateau-hedra.}

A mean curvature convex polyhedron $P \subset X$, e.g. a convex polyhedron in $\mathbb{R}^{n}$, is called dihedrally extremal if no "deformation" $P \leadsto P^{\prime}$ of $P$ can diminish its dihedral angles while keeping the faces mean curvature convex. (Compare $\$ 5 \frac{4}{9}$ in [19.)

That is, more precisely, if a mean curvature convex polyhedron $P^{\prime} \subset X$ of the same combinatorial type as $P$ has (non-strictly) smaller suprema of its dihedral angles along all $(n-2)$-faces $Q_{i}^{\prime} \cap Q_{j}^{\prime} \subset P^{\prime}$ than $P$,

$$
\sup _{Q_{i}^{\prime} \cap Q_{j}^{\prime}} \angle\left[Q_{i}^{\prime} \pitchfork Q_{j}^{\prime}\right]=\alpha_{i j}^{\prime} \leq \alpha_{i j}=\sup _{Q_{i} \cap Q_{j}} \angle\left[Q_{i} \pitchfork Q_{j}\right],
$$

then, necessarily, $\alpha_{i j}^{\prime}=\alpha_{i j}$.

$P P$-hedra. An polyhedral domain $P \subset X$ is called a (poly) Plateau-hedron, or PP-hedron if all its $(n-1)$-faces have zero mean curvatures and the dihedral angle functions $\angle_{i j}=\angle\left[Q_{i} \pitchfork Q_{j}\right]$ are constant on the edges that are $(n-2)$-faces $Q_{i j}=Q_{i} \cap Q_{j}$ for the pairs of $(n-1)$-faces $Q_{i}, Q_{j} \subset P$ with $\operatorname{dim}\left(Q_{i} \cap Q_{j}\right)=n-2$.

Notice that we do not require preconvexity of $P$ (which is equivalent to mean curvature convexity in this case) but we will be dealing mainly with preconvex PP-hedra.

Basic instances of Plateau-hedra are ordinary polyhedral domains with flat (i.e. totally geodesic) faces in manifolds $X$ of constant curvature, e.g. in $X=\mathbb{R}^{n}$.

Also it is easy to see that

dihedrally extremal mean curvature convex polyhedra $P$ in Riemannian manifolds are Plateau-hedra.

Singularities: Cones and Corners. The above does not imply, however, that all combinatorial types of polyhedra contain dihedrally extremal representatives, since the corresponding existence/regularity theorem is unavailable.

On the other hand, one may attempt a construction of Plateau-hedra in a Riemannian manifold $X$ by a variational argument where a face $W=W_{i}$ of a desirable $P$ is obtained as a solutions of a Plateau type problem with free boundary, i.e. the boundary of $W$ must be contained in the union $W_{\perp}=W_{\hat{i}}$ of the remaining faces, see section 3 and 4 .

Yet, such a $W$ may have singularities, both in the interior and at boundary points $x$ in $W$.

For instance, if $n-1=\operatorname{dim}(W) \geq 7$, then $W$ may have quasi conical singularities at some points $x \in W$ where, by definition, a tangent cone is non-flat.

But if $x \in \operatorname{int}(W)$, or if $x \in \partial W \cap \operatorname{reg}\left(W_{\perp}\right)$, i.e. if $x$ lies away from the edges of $W_{\perp}$ as well as from interior singularities of the faces of $W_{\perp}$ and if a tangent cone of $W$ at $w$ is flat, then $W$ is smooth at $w$, see [1 [26, 29, 27, 1]

But the behaviour of $W$ at the singular points of $W_{\perp}$, even at the regular corners, i.e. where $\partial W$ meets edges between non-singular faces in $W_{\perp}$, may be more complicated, e.g. see [47, [6].2]

\footnotetext{
1 This was explained to me by Fang-Hua Lin. Also I had a useful communication with Frank Morgan and Brian White concerning these problems.

${ }^{2}$ I must admit I have not truly studied these papers.
} 
In particular, one has the following

Perturbation Question. Let $P_{0} \subset \mathbb{R}^{n}$ be a cosimpicial convex polyhedron. Does it admit an arbitrary small perturbation to a Plateau-hedron $P^{\prime}$ with non-flat faces?

A natural approach here would be via a solution of the linearized problem combined with the implicit function theorem, but one can not guarantee regularity at the corners 3

Inevitability of singularities suggests a more general definition of Plateauhedra and of cornered domains in general and such a concept is also needed in the (conjectural) context of the theory of singular spaces with scalar curvatures bounded from below. But our understanding of singular polyhedra, in particular of singular Plateau-hedra, remains unsatisfactory.

Why at the Corners? The simplest instance of singularities at the corners is that of minimal surfaces in $Y \subset \mathbb{R}^{3}$ contained in intersection $P$ of two subspaces with free boundary $\partial Y \subset \partial P$. If the (dihedral) angle $\alpha$ between the half plane that make the boundary of $P$ is $\pi / k$ for an integer $k$ then $Y$ extends by reflections to a minimal hypersurface, say $2 k Y$ around the edge in $P$. Consequently, $Y$ is smooth, actually real analytic, in this case.

Furthermore, because of the $2 k$-th order symmetry, $2 k Y$, and hence $Y$ as well, are flat of order $k$ at the corner point $y_{\llcorner} \in Y$, i.e. where $Y$ it meets the edge in $P$.

But if $\alpha$ is incommensurable with $\pi$, the same symmetry argument shows that if smooth, then $Y$ must be flat of infinite order at $y_{\llcorner}$. In particular $Y$ can not be real analytic unless it is flat and, probably,(?) it cannot be even $C^{2}$.

On the other hand if a curve-faced $P$ has the dihedral angle $\pi / k$ one expects a reasonable smoothness at the corner.

\subsection{Euclidean Dihedral Extremality Problem.}

[?] Convexity $\Leftrightarrow$ Dihedrally Extremality [?]

Namely,

$[\mathbf{C O N V} \Rightarrow$ DEXT? $] \quad$ Which convex polyhedra $P \subset \mathbb{R}^{n}$ are dihedrally extremal?

$[\mathbf{D E X T} \Rightarrow \mathbf{C O N V} ?] \quad$ Are there non-convex dihedrally extremal $P \subset \mathbb{R}^{n}$ ?

My guess is that $[\mathbf{D E X T} \Rightarrow \mathbf{C O N V}$ ] for all combinatorial types $\mathcal{C} \mathcal{T}$ of $P$, i.e. all m.c. dihedrally extremal m.c. convex polyhedral domains in $\mathbb{R}^{n}$ are convex, even if we allow the above mentioned singularities.

On the contrary, dihedral extremality seems too good to be true for all convex polyhedra $P \subset \mathbb{R}^{n}$.

In fact, even if $P^{\prime} \subset \mathbb{R}^{n}$ is a convex (not just mean curvature convex) polyhedron, combinatorially equivalent to $P$, it is unclear why the dihedral angles of $P^{\prime}$ can not be all strictly smaller than the corresponding angles in $P$. This can not happen for simplices $P=\Delta^{n} \subset \mathbb{R}^{n}$ by Kirszbraun theorem applied to the dual simplices $\left(\Delta^{n}\right)^{*}$

\footnotetext{
3 The existence of singularities for linear boundary value problems in domains with edges and corners was pointed out to me by Jeff Cheeger and the full extent of the difficulty of this problem was explained to me by Vladimir Mazia.
} 
Possibly, simplices and their Cartesian products are dihedrally extremal. Also we have the following

$\triangle \times \square \times \square \times \square \times \ldots$-Conjecture. The Cartesian products $P \subset \mathbb{R}^{2 k+m}$ of $k$ convex polygones $\subset \mathbb{R}^{2}$ and an $m$-cube $\subset \mathbb{R}^{m}$ are m.c. dihedrally extremal: one can not diminish the dihedral angles by "deforming" such a $P$ without developing negative mean curvature in some of the faces.

Theorem-Example: $\Delta \times \square \times 0$-Extremality. The simplest case where we settle this conjecture is for Cartesian products of $m$-cubes with Cartesian powers of regular triangles and regulate hexagons,

$$
P=\square^{m_{1}} \times \Delta^{m_{2}} \times \square^{n_{3}} \subset \mathbb{R}^{2 m_{3}+m} .
$$

In fact, (see "Gluing around Edges" in section 2) this $\Delta \times \square \times 0$-extremality follows from the positive solution to the Geroch conjecture on non-existence of metrics of positive scalar curvatures on tori $\mathbb{T}^{n}$.

Strangely enough, there is no apparent direct elementary proof of this apparently intrinsically Euclidean inequality/extremality.

There are, recall, two approaches to the Geroch conjecture. The original one, due to Schoen and Yau, depends on smoothness of minimal hypersurfaces $H^{n-1} \subset \mathbb{T}^{n}$ and applies only to $n \leq 7$. Their argument easily extends to $n=8$ by non-stability of singularities of 7-dimensional minimal hypersurfaces [44, while a way around singularities for $n \geq 9$, found relatively recently by Lohkamp [35,

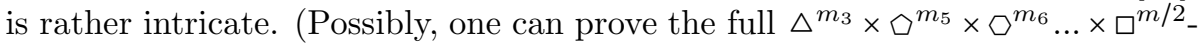
conjecture utilizing Lochkamp's techniques.)

Another proof (see [23]), that depends on the index theorem for twisted Dirac operators, indiscriminately applies to all $n$, but it needs the spin structure. (This causes no problem for $\mathbb{T}^{n}$ but becomes a hurdle for non-spin manifolds $X$.)

We shall interpret applications of these methods to corned domains $P$ as "billiard games" played by Dirac operators and minimal hypersurfaces in $P$ (see section 2.3).

Extremality, Rigidity and Scalar Curvature. The concepts of the dihedral extremality as well as the extremality of manifolds with positive scalar curvature that was studied in [19] [41, 32, [12] are embraced by the following

Definition. Let $X=(X, g)$ be a Riemannian $n$-manifold $X$ with corners, let $f$ be a smooth map of non-zero degree of another smooth $n$-manifold $Y$ onto $X$ and let us endow $Y$ with a corner structure induced by $f$ from $X$. (One may admit $Y$ with $\operatorname{dim}(Y) \geq \operatorname{dim}(X)$ with a suitable concept of degree for maps $f: Y \rightarrow X$.)

$X$ is called extremal if every for every such map $f$ every Riemannian Let $Y$ be endowed with a Riemannian metric $g_{Y}$ such that

(1) $f$ is distance decreasing (sometimes one only requites $f$ being area decreasing);

(2) $f$ decreases the scalar curvature:

$$
\operatorname{scal}_{X}(f(y)) \leq \operatorname{scal}_{Y}(y) \text { for all } y \in Y \text {; }
$$

(3) $f$ decreases the mean curvature of the $(n-1)$-faces:

$$
\text { mean.curv }(f(y)) \leq \text { mean.curv }(f(y)) \text { for all } y \text { in all }(n-1) \text { faces of } Y \text {; }
$$


(4) $f$ decreases the dihedral angles at all edgers

$$
\left\llcorner_{f(y)} \geq \angle_{y} \text { at all } y \text { in all }(n-2) \text {-faces of } Y\right. \text {; }
$$

Then $X$ is called extremal, if for no such $Y, f$ and $g_{Y}$ the increases/decreases in the inequalities (2)-(4) can be strict, not at a single point $y \in Y$ while rigidity says, not quite precisely, that all $Y$ satisfying (1)-(4) are obtained from $X$ by "obvious modifications".

Notice that (1)-(3) can be unified if the $\operatorname{scal}_{X}(x)$ is understood as the mean curvature at the points $x$ in the (interiors of) $(n-1)$-faces of $X$ as $\pi-\angle_{x}$ at $x$ in the $(n-2)$-faces.

(A) Several extremality/rigidity results are available for closed (i.e. of depth 0 ) manifolds with positive scalar curvature, in particular for most (all?) compact Riemannian symmetric spaces $X$, see [41, 32, 12], 13, 34, 19] which is proved with Dirac operators.

Can this be proved by means of minimal hypersurfaces or, rather, of $\phi$-bubbles (see 3.1)?

The extremality/rigidity of the round spheres $[34$ implies that convex metric balls in simply connected spaces $X$ of constant curvatures ("convex" is relevant if $\operatorname{curv}(X)>0$, i.e. $X=S^{n}$ ) are extremal for $n=\operatorname{dim}(X) \leq 8$. This follows by the wrapped product argument from $\S 5 \frac{5}{6}$ in [19, where the case $n=8$ relies on non-stability of (isolated) singularities of $\phi$-bubbles in 8-manifolds (as well as of minimal hypersurfaces [44) and where, possibly, the extremality, but not, a priori, rigidity may be obtained with [35].

This rigidity is reminiscent of the generalized positive mass theorem [41] and suggests a possibility of proving this extremality/rigidity by the Dirac operator method.

(B) The simplest examples of extremal/rigid $P$ are convex $k$-gons in surfaces of positive (not necessarily constant) curvatures, where their extremality and rigidity follows from the Gauss-Bonnet theorem.

Are Cartesian products of extremal/rigid manifolds, in particularly of those in the above examples (A) and (B), extremal/rigid?

We prove in this regard the rigidity of $3 D$-prisms $\left(k\right.$-gons $\left.\times[0, r] \subset \mathbb{R}^{3}\right)$ in section 5.4.

Probably, the rigidity of Cartesian products of $k$-gons (at least in the spin case) follows by extending the methods of [13] to manifolds with singularities along codimension two (divisor-like) subvarieties, where the relevant examples are Cartesian products of surfaces with isolated conical singularities.

(C) Some (but not all) warped products of Riemannian manifolds may be extremal/rigid.

For instance, let $X=Y \times[0, R]$, where $Y \subset \mathbb{R}^{n-1}$ is a convex polyhedral domain and where the warped product metric $g(y, t)=a(t)^{2} g(y)+d t^{2}$ on $X$ has constant negative curvature, namely, $a(t)=e^{t}$.

Does extremality/rigidity of $Y$ imply that of $X$ ?

In fact, the rigidity of $X$ is proved in [19] for $Y$ being a flat torus of dimension $n-1 \leq 6$, where the extremality (but not rigidity) for $n-1=7$ extends with [44] and, probably, with 35] to all $n$. 
The $\phi$-bubble argument from 19 can be combined with gluing around the edges (see 2.1) thus proving the extremality/rigidity of these warped product $X$ for Euclidean reflection domains $Y$, at least for $\operatorname{dim}(Y) \leq 6$.

Can one prove the rigidity/extremality of these warped products by a pure Dirac operator method in the spirit of [40]?

(D) Annuli $X=S^{n-1} \times[0, r]$ between concentric spheres in manifolds of constant curvature are instances of warped products which have geometric properties similar to but different from our extremality/rigidity.

Such properties are proved in 19 by means of $\phi$-bubbles that limits the results to $n \leq 7$ (extended to $n \leq 8$ with [44] and, possibly, to all $n$ with [35]).

Similar properties may be true for some (e.g. reflection, that is no big deal) domains $P \subset S^{n-1}$ but the overall picture is far from clear.

Observe, finally, that if a certain space $X$ (without mean convexity points at its boundary) of negative scalar curvature is "extremal", this extremality must be opposite to what we saw above: when one enlarges such an $X$ its scalar curvature tends to increase rather then decrease.

In other words, the distance decreasing condition for maps $f: Y \rightarrow X$ is satisfactory restrictive if $\operatorname{scal}(X) \geq 0$ but it seems more logical to require $f$ to be distance increasing (which needs to be properly defined for non-injective maps) at the points where $s c a l \leq 0$.

Ideally, one wants to prove extremity and rigidity relative to maps $f: Y \rightarrow X$ that decrease the integrals of the scalar curvatures over some class of surfaces in the two manifolds, something like $\int_{S} \operatorname{scal}(Y) d s \leq \int_{T} \operatorname{scal}(Y) d t$ for $T \subset Y$ and $S=f(T) \subset X$ for the same kind of surfaces of $S$ and $T$ as in the semiintegral inequalities in section 5.4 of the present paper and and in 0.5.C of [17].

\subsection{On Acute Polyhedra.}

Besides products of $k$-gons, there is another class of "elementary" polyhedral domains where one may expect extremality/rigidity results.

Call a Riemannian manifold $P$ with corners (non-strictly) acute if all its dihedral angles are acute, i.e. bounded by $\pi / 2$,

$$
\angle\left[Q_{i} \pitchfork Q_{j}\right] \leq \pi / 2 \text { for all pairs of adjacent }(n-1) \text {-faces } Q_{i}, Q_{j} \subset P \text {. }
$$

Acute Spherical Polyhedra. If a convex spherical polyhedron $P \subset S^{n}$ (i.e. an intersection of hemispheres) has acute dihedral angles then it is a simplex, or, in the degenerate case, the spherical suspension over a simplex in $S^{n-i} \subset S^{n}$.

Indeed, the dual polyhedron, say $P_{p}^{\perp} \subset S^{n}$, has all its edges longer than $\pi / 2$. Consequently, the distance between every two vertices in $P_{p}^{\perp}$ is $\geq \pi / 2$; hence, there are at most $n+1$ vertices in $P_{p}^{\perp}$.

It follows, that

acute Riemannian manifolds $P$ with corners are simple - there are exactly $n-i$ faces $Q_{i}^{n-1}$ transversally meeting along every $i$-face $p \in \partial(P)$.

Also observe that (non-strictly) acute spherical triangles $\triangle \subset S^{2}$ have all their edges bounded in length by $\pi / 2$. It follows that all $m$-faces, $m=2,3, \ldots, n-$ 1 , of acute spherical $n$-simplices are acute.

Acute Euclidean Polyhedra. Cartesian products of acute simplices $\triangle^{n_{i}} \subset \mathbb{R}^{n_{i}}$,

$$
P=\triangle^{n_{1}} \times \triangle^{n_{2}} \times \ldots \times \Delta^{n_{k}} \subset \mathbb{R}^{n_{1}+n_{2}+\ldots+n_{k}},
$$


are, obviously, acute.

Conversely,

every acute polyhedron $P \subset \mathbb{R}^{n}$ is a Cartesian product of simplices.

This is easy and, certainly, has been known for ages. But I could not find this on the web and wrote down a (few lines) proof in 21 .

Questions. Are all acute polyhedron $P \subset \mathbb{R}^{n}$ dihedrally rigid or at least external?

What are possible combinatorial types of mean curvature convex Riemannian cornered manifolds $P$ with $\operatorname{scal}(P) \geq 0$ and acute dihedral angles?

Are there any constrains on the combinatorial types of mean curvature convex $P \subset \mathbb{R}^{n}$ and $\angle\left[Q_{i} \pitchfork Q_{j}\right] \leq(\pi / 2)+\alpha$ for a given $0<\alpha<\pi / 2$ ?

(I stated in my article Hilbert Volume in Metric Spaces that there are only finitely many combinatorial types of convex polyhedra with

$$
\angle\left[Q_{i} \pitchfork Q_{j}\right] \leq(\pi / 2)+\alpha, \alpha<\pi / 2,
$$

but Karim Adiprasito recently showed me counterexamples starting from dimension 3.)

On the other hand, the scalar curvature can be made arbitrarily large by multiplying any (compact) $P^{n-2}$ by a small 2-sphere, where $P^{n}=P^{n-2} \times S^{2}(\rho)$ has the same dihedral angles and mean curvatures of the faces as $P^{n-2}$, but these have "rather degenerate" combinatorial types.

One may expect that some (most?) manifolds $P$ with corners (convex polyhedra?) support no metrics $g$ with $\operatorname{scal}(g) \geq 0$, with mn.curv $_{g}\left(Q_{i}\right) \geq 0$ and with $\angle_{g}\left[Q_{i} \pitchfork Q_{j}\right] \leq(\pi / 2)+\alpha$ for every $\alpha<\pi / 6$ and, less likely, for $\alpha \geq \pi / 6$.

Yet, finding a single such $P$ for any $\alpha>0$ remains problematic.

Also we can not solve the following

Simplex Problem. Let $P \subset \mathbb{R}^{n}$ be a curve-faced polyhedral domain that is combinatorially equivalent to the $n$-simplex and let $\alpha_{\max }(P)$ denote the supremum of its dihedral angles at all edge points. Notice that if $n \geq 3$ then, obviously, $\alpha_{\max }(P)>\pi / 3$.

What is the infimum of $\alpha_{\max }(P)$ over all mean curvature convex $P$ ?

Conjecturally, this inf $\alpha_{\max }$ is assumed by (the dihedral angle between a pair of faces of ) the regular $n$-simplex with flat faces, but it is not even a priori clear if inf $\alpha_{\max }$ is strictly greater than $\pi / 3$ for $n \geq 3$.

\section{8 $C^{0}$-Limits of Metrics with $s c a l \geq \kappa$.}

Our study of mean curvature convex polyhedral domains in Riemannian manifolds $X$, even for $X=\mathbb{R}^{n}$, is intimately related to the scalar curvature. For example we shall prove the extremality of the above $P$ in the class of all spin manifolds $P^{\prime}$ with corners which have $\operatorname{scal}(X) \geq 0$ by utilizing minimal hypersurfaces along with Dirac operators.

We also achieve this for non-spin manifolds $X$ with $\operatorname{dim}(X) \leq 9$, where the singularities of minimal hypersurfaces are at most 1-dimensional (actually, we shall need this for "Plateau bubbles" in $X$, see 5.3) and, as we will show, they do not "feel" spin obstruction that lives in dimension 2. Probably, the analysis of singularities developed in 35. would allow a direct (with no use of spinors) proof for all $\operatorname{dim}(X)$. 
Most current results on manifolds $X$ with $\operatorname{scal}(X) \geq 0$ rely on global techniques and do not tell you much on the geometry of small (but not infinitesimally small) and moderately large regions $U \subset X$. For example, the Dirac operator can be directly used (almost) exclusively on complete manifolds $X$ (an exception is Min-Oo rigidity theorem for the hemisphere [41]) while the Schoen-Yau approach depends on a presence of closed/complete (or "quite large" as in 25]) minimal hypersurfaces $Y^{n-1} \subset X=X^{n}$, similarly to how the proof Synge's theorem for manifolds $X$ with sect.curv $(X)>0$ uses closed geodesics in $X$.

Sometimes, one can derive semi-global results from global ones, either by extending a metric from a manifold $X$ with a boundary (or such a domain $U \subset X)$ to a complete $X_{+} \supset X$ keeping $\operatorname{scal}\left(X_{+}\right) \geq 0$ [11 or by exploiting Plateau "soap" bubbles $Y^{n-1} \subset X[19$ to which global techniques apply.

Yet, all this fails short of Alexandrov's approach to spaces with sect.curv $\geq 0$ (and more generally with sect.curv $\geq-\kappa^{2}$ ) via (comparison) inequalities for angles of geodesic triangles that indiscriminately hold on all scales and provide a non-trivial information on the geometry of all domains $U \subset X$, be they big or small.

Hopefully, lower bounds on dihedral angles of extremal PP-hedra $U \subset X$ may play a similar role for $\operatorname{scal}(X) \geq 0$. This, in turn, points toward an Alexandrov's type theory of singular spaces $X$ with $\operatorname{scal}(X) \geq 0$ and, possibly, with $\operatorname{scal}(X) \geq$ $-\kappa^{2}$.

Notice that there is an analytic approach to singular metrics with positive scalar curvature understood in the distribution sense in [14 and somewhat similar in 31 but these do not seem to apply to our situation.

We do not know what the theory of objects (spaces?) with positive scalar curvatures understood in the distribution sense should be but we prove in section 4.9 the following

$C^{0}$-Limit Theorem. (Compare $\S 5 \frac{5}{6}$ in [19]) Let a smooth Riemannian metric $g$ on a Riemannian manifold $X$ equal the uniform limit of smooth metrics $g_{i}$ with $\operatorname{scal}_{g_{i}}(x) \geq \kappa(x)$ for a continuous function $\kappa$ on $X$. Then $\operatorname{scal}_{g}(x) \geq \kappa(x)$ as well..

Remarks, Questions, Speculations. (a) The above is a local property of metrics and the general case trivially follows from that where $\kappa$ is constant.

The $C^{0}$-limit property for $\kappa=0$ is derived from the existence of particular (small) strictly mean convex cubical domains with acute dihedral angles in manifolds with scal $<0$ (see ( $\square$ ) in 4.9) and the solution to the Geroch conjecture on non-existence of metrics with scal $>0$ on tori, while the cases $\kappa>0$ and $\kappa<0$ reduce to $\kappa=0$ as follows.

First, let $\kappa=n(n-1)$ for $n=\operatorname{dim}(X)$, where observe this $\kappa$ equals the scalar curvature of the unit Euclidean $n$-sphere $S^{n}$.

Given a metric $g$ on $X$ let $\check{X}=\left(X \times \mathbb{R}_{+}, \check{g}\right)$ be the standard Riemannian/Euclidean cone over $(X, g)$, that is $g(x, t)=t^{2} g(x)+d t^{2}$.

Notice that if $\operatorname{scal}_{g}(x)=n(n-1)$ at a point $x \in X$, then $\operatorname{scal}_{\breve{g}}(x, t)=0$ and if $\operatorname{scal}_{g}(x)<n(n-1)$, then $\operatorname{scal}_{\breve{g}}(x, t)<0$ for all $t>0$.

Thus, the $C^{0}$-limit theorem for metrics on $X$ with $\kappa=n(n-1)$, hence, for all $\kappa>0$, reduces to that for $\kappa=0$ on $X \times \mathbb{R}_{+}$.

(If $X$ is a compact manifold with scal $>0$ then the double $2 \diamond \check{X}$ of the cone $\check{X}=X \times \mathbb{R}_{+}$at the vertex corresponding to $\mathrm{t}=0$ admits a complex metric of positive curvature that is conical at both ends of this double. This suggests that 
geometric properties of such $X$ can be expressed and/or generalized in terms of asymptotic geometries of complete manifolds where Witten and Min-Oo style spinor arguments may be applicable. Can one, for instance, derive Llarull's sphere rigidity theorem along these lines?)

Now let $\kappa<0$ and proceed similarly albeit more artificially. Namely, let $\hat{X}=(X \times[-\delta, \delta], \hat{g})$ for $\hat{g}(x . t)=C_{\kappa} t^{2} g(x)+d t^{2}$, where the constant $C_{\kappa}>0$ is chosen such that $\operatorname{scal}_{g}(x)=\kappa \Rightarrow \operatorname{scal}_{\hat{g}}(x, 0)=0$ for a given $\kappa<0$.

Then the inequality $\operatorname{scal}_{g}(x)<\kappa$ implies $\operatorname{scal}_{\hat{g}}(x, 0)<0$ and the $C^{0}$-approximation theorem on $X$ with $\kappa<0$ is thus reduced to that on $\hat{X}$ with $\kappa=0$.

(The natural cone metric in $X \times \mathbb{R}_{+}$where $\kappa(X)<0$ is a Lorentzian one to which our flat Riemannian argument does not(?) apply; yet, 40] suggests an approach to this metric.)

Conclude by noticing that if one is willing to add two (or more) extra dimensions one can reduce the case of $\kappa \neq 0$ to that of $\kappa=0$ by taking the Riemannian product $X \times D^{2}$ for a disc $D^{2}$ with $\operatorname{scal}\left(D^{2}\right)=-\operatorname{scal}(X)$.

Thus, the relevant information concerning $X$ may be seen in the geometry of the torus $T^{n+2}$ that is obtained by reflection development (orbicovering, see 2.1 ) of a small cube in $X \times D^{2}$ (see () in section 4.9) where the sections of this cube by $X \times d, d \in D^{2}$ make an $n$-dimensional foliation.

Can one abstractly define and study this kind of foliations with no reference to any external $D^{2}$ ?

(b) The $C^{0}$-limit theorem contrasts with Lokhamp's h-principle:

every Riemannian metric $g$ on a smooth manifold $X$ can be $C^{0}$-approximated by metrics $g_{i}$ with scal $\left(g_{i}\right)<0$ and even with Ricci $\left(g_{i}\right)<0$.

(c) If $n=\operatorname{dim}(X)=3$, then the $C^{0}$-limit theorem for all $\kappa$, be they positive or negative, follows, from the Gauss-Bonnet prism inequality in 5.4. and a version of ( $\square$ ) from 4.9 (where it is used for the case $\kappa=0$ ) adjusted to $\kappa \neq 0$.

(d) The $C^{0}$-limit theorem for $\kappa<0$ can be also proven in a more natural fashion intrinsically in $X$ itself similarly to the case $\kappa=0$ with a version of () for bands around (germs of) suitable convex hypersurfaces $Y$ in $(X, g)$ with induced metrics having scalar curvatures zero (or, rather, close to zero) by reproducing the argument presented at the end of $\S 5 \frac{5}{6}$ in [19] with small cubical $P \subset Y$ instead of $(n-1)$-tori as in 19]. (These $P$ are similar to hyperbolic $n$-dimensional "prisms" that are suspended over reflection domains in $Y_{h o r o}^{n-1}$ discussed toward the end of section 5.4.)

(e) The $C^{0}$-limit theorem is reminiscent of Eliashberg's $C^{0}$-closeness theorem for symplectic diffeomorhisms.

Is there something in common between the two theorems besides superficial similarity?

Is there a scalar curvature counterpart of Hofer metric between Hamiltonian diffeomorphisms and/or Lagrangian submanifolds?

Is there anything interesting (besides a specific $K$-area inequality from $\S 5 \frac{4}{5}$ in [19]) in geometry of quasi-Kählerian metrics with positive scalar curvatures on symplectic manifolds?

Also, the $C^{0}$-closeness of scal $\geq \kappa$ resembles Novikov's theorem on the topological invariance of Pontryagin classes, but it is equally unclear if there is something profound behind this similarity. 


\section{$1.9 \quad$ Rigidity Problems around scal $\geq 0$.}

Let a continuous Riemannian metric $g$ on a closed manifold $X$ admits a $C^{0}$ approximation by smooth metrics $g_{i}$ with $\operatorname{scal}\left(g_{i}\right) \geq-\varepsilon_{i} \rightarrow 0, i \rightarrow \infty$.

$\bullet_{1}$ Does $X$ admit a smooth metric $g^{\prime}$ with $\operatorname{scal}\left(g^{\prime}\right) \geq 0$ ?

$\bullet_{2}$ Suppose that $X$ admits a continuous map to the $n$-torus, $n=\operatorname{dim} X$, of non-zero degree. Is then the metric $g$ itself necessarily flat?

One asks similar questions concerning Dirac operators $D=D_{g}$, say on spin manifolds $X$ :

Is the spectrum of $D^{2}=D_{g}^{2}$ semi-continuous for the $C^{0}$-topology in the space of smooth Riemannian metrics $g$ ?

In particular, let $g_{i} \underset{C^{0}}{\rightarrow} g$, where $g_{i}$ are smooth metrics with positive squared Dirac operators $D_{g_{i}}^{2}$.

If $g$ is smooth, is then $D_{g}^{2}$ also (non-strictly) positive?

What are classes of continuous metric $g$ on an $X$ where positivity of $D_{g}^{2}$ makes sense? (A particular class of interest is that of piece-wise smooth metrics on closed manifolds obtained by gluing Plateau-hedra along their faces, where the approach from [39, 37, 31] and [14] may be relevant.)

Also one may raise such questions for other geometric operators, e.g. for the coarse Laplacians in the bundles associated to the tangent bundle, where "spectral $C^{0}$-semi-continuity" of such operators is related via the Feynman-Kac formula to the following question.

$C^{0}$-Semi-continuity of Holonomy. Let $g_{i} \underset{C^{0}}{\rightarrow} g$, where $g_{i}$ are smooth metrics on the $n$-dimensional manifold $X$ with their holonomy groups contained in a given closed subgroup $H \subset O(n)$ in the linear group acting on the tangent space $T_{x_{0}}(X)$. Is the holonomy group of $g$ also contained in $H$ ?

This is, probably, not hard to prove for "classical $H$ ", e.g. for $H=O(k) \times$ $O(n-k)$, where the corresponding manifolds $\left(X, g_{i}\right)$ split; thus, carry many flat geodesic subspaces. One may approach in a similar fashion holonomy groups of symmetric spaces and, possibly, of Kähler manifolds.

Is there a metric characterization of Kähler manifolds that is stable under $C^{0}$-limits of metrics similar to that in [18] but that, unlike [18] would apply to non-necessarily closed manifolds, e.g. to (small) domains in projective algebraic manifolds?

\subsection{Singular Spaces with $s c a l \geq 0$ and Related Problems.}

A potential pool of singular $X$ with $\operatorname{scal}(X) \geq 0$ spreads immensely wider than that or than the class of the Alexandrov spaces with sect.curv $\geq 0$. In particular, this "pool" must include:

- spaces partitioned into "PP-hedral cells" with essentially conical singularities where the local geometry is similar to that for sect.curv $\geq 0$,

- certain spaces with "fractal singularities".

Here are two such examples.

(1) Let $\operatorname{scal}(X) \geq 0$ and $U \subset X$ be a domains $U \subset X$ where the boundary $\partial U$ of $U$, that is allowed to have singularities, has non-negative mean curvature, e.g. where $\partial U$ comes as a minimal hypersurface in $X$. Then the double of $U$ 
along $\partial U$ must be regarded as a space with scal.curv $\geq 0$. In fact, such an $X$ is often (always?) equals Hausdorff limit of smooth manifolds with scal $\geq 0$

(2) Let $X_{1}$ and $X_{2}$ be $n$-manifolds and $Y_{i} \subset X_{i}, i=1,2$ be submanifolds for which there exists a diffeomorphism $Y_{1} \leftrightarrow Y_{2}$ that induces an isomorphism of their respective normal bundles.

This delivers a diffeomorphism between the boundaries of the normal neighbourhoods $U_{i}^{n r m} \supset Y_{i}$, call it $\chi: \partial U_{1}^{n r m} \leftrightarrow \partial U_{2}^{n r m}$, and let $X=X_{1} \#_{\chi} X_{2}$ be obtained by gluing $X_{1} \backslash U_{1}^{n r m}$ with $X_{2} \backslash U_{2}^{n r m}$ according to $\chi$.

If $\operatorname{scal}\left(X_{i}\right)>0$ and $\operatorname{codim}\left(Y_{i}\right) \geq 3$, then this $X$ carries a canonical class of metrics with scal $>0$ that equal the original ones on $X_{i} \backslash U_{i}^{n r m}$.

This gluing operation can be repeated infinitely many times and the resulting limit spaces should be regarded as having $s c a l \geq 0$ with the simplest instance of this is as follows.

Let $X_{0}=X_{0}^{n}, n \geq 3$, be a compact manifold with $\operatorname{scal}\left(X_{0}\right)>0$ and $\left\{y_{1}, y_{2}, y_{3}\right\} \subset$ $X$ be a 3-point subset. Let us attach to each of these points a copy of $\zeta \cdot X_{0}$, that is $X_{0}$ with the metric scaled by $\zeta>0$, where the point in $\left(\zeta \cdot X_{0}\right)_{i}$ that corresponds to $y_{1} \in X_{0}$ is attached to $y_{i} \in X_{0}$, for each $i=1,2,3$. The resulting manifold $X_{1}$ (that is the connected sum of $X_{0}$ and three copies of $\zeta \cdot X_{0}$ ) has six "free" points corresponding to the unused counterparts of $y_{2}$ and $y_{3}$ in the three $\zeta \cdot X_{0}$.

Let $X_{2}$ be obtained by attaching a copy of $\zeta^{2} \cdot X_{0}$ to $X_{1}$ at each of these points and then, similarly, we get $X_{3}, X_{4}$, etc..

If $\zeta>1$, the the Hausdorff limit, call it $(1-\zeta)^{-1} * X$, of the resulting sequence of spaces $X_{0}, X_{1}, X_{2}, \ldots$ is a smooth complete non-compact Riemannian manifold. If $\lambda<1$, this is a compact self-similar fractal space $X$ which behaves in many respects as nicely as Riemannian manifolds do (e.g. it may have essentially Euclidean filling inequalities see section 4.7) and it definitely must be regarded as having $\operatorname{scal}(X)>0$.

Also notice that if $\zeta^{n}<1 / 2, n=\operatorname{dim}\left(X_{0}\right)$, then the volume of $(1-\zeta)^{-1} * X$, that is $\approx \sum_{i} 2^{i} \operatorname{vol}\left(\zeta^{i} \cdot X_{0}\right)=\operatorname{vol}\left(X_{0}\right) \sum_{i} 2^{i} \zeta^{n i}$, is finite.

Remarks. (a) The class of spaces with scal $\geq 0$, unlike that with sect.curv $\geq 0$, can not be stable under Hausdorff limits, unless extra strong "topological noncollapsing" conditions are imposed on the spaces involved. These conditions can be enforced in the above case where $X$ equals the Hausdorff-Lipschitz projective limit of $X_{i}$ for naturally defined uniformly Lipschitz maps $X_{i+1} \rightarrow X_{i}$.

(The classes of spaces considered in [5], 39, 37, 31] and [14 do not seem to be stable under geometric limits.)

(b) On scal $\geq \sigma$. A lower bound on the scalar curvature by a positive or negative constant $\sigma \neq 0$ is not scale invariant and geometric characteristic of the corresponding manifolds must include a bound on their "size" and/or a lower bound on the mean curvatures of their boundaries (if there are any)including faces of of spherical and hyperbolic polyhedra $P$, if we want to prove their extremality.

(c) Besides scal $>0$, there are other classes of Riemannian metrics that are stable under geometric connected sums of manifolds. The most prominent among these are conformally flat metrics (where one may simultaneously keep positivity of scal if one wishes) and metrics with positive isotropic curvature. This curvature is defined in terms of the complexified tangent bundle of $X$ and its positivity may be expressed in writing by $K_{\mathbb{C}}(X)>0$, 38. 
Probably, suitable limits of such connected sums can be embraced by a general theory that would allow singular spaces.

Sample Question. Is there a natural class of singular spaces $X$ with $K_{\mathbb{C}}(X)>$ 0 that would satisfy (a suitable version of) the Micallef-Moore [38] and/or La Nave [30] bounds on indices and sizes of harmonic maps of surfaces into $X$ ?

More Questions. What is the geometry of piecewise smooth Riemannian metrics?

Manifolds with such metrics (which are not, in general, Alexandrov as they may have minus infinite sectional curvatures at their $(n-1)$-faces) seem to support full-fledged Dirac operators and allow solutions of the Plateau problem.

Limit/Closure Question. What are natural limits of such spaces retaining their essential "nice" properties.

The spaces we expect to have among such limits should include the doubles of domains $P$ with boundaries in smooth manifolds, where the boundaries $\partial P$ may have certain singularities. For example, these $\partial P$ may be (stable?) minimal hypersurfaces with singularities.

Approximation Question. When does a Riemannian metric $g$ on $X$ admit an approximation by piece-wise smooth ones, where

- the interiors of all pieces are flat (or, more generally have a given constant curvature),

-' the exterior curvatures of all $(n-1)$-faces (looked from the interiors of the corresponding $n$-faces) satisfy some convexity condition,

-" the sums of the dihedral angles between $(n-1)$-faces around all $(n-2)$ faces are bounded by $2 \pi$ ?

For example, let $S c(g) \geq 0$. Can one approximate it with $\bullet$ signifying positive mean curvatures?

This, possibly, can be done (at least for $S c>0$ ) by starting with a fine triangulation of $X$ into fat simplices with almost flat faces, and then flattening the insides of these $n$-simplices and, at the same time, gaining the mean curvature convexity of their $(n-1)$-faces while keeping the total angles around the $(n-2)$ faces equal $2 \pi$ )

(If sect.curv $\geq 0$, then one may ask for convexity instead of mean convexity but this seems less realistic.)

\section{Reflection Domains.}

An $n$-manifold $P$ with corners is called a $\Gamma$-reflection domain if it is represented as a fundamental domain of a discrete reflection group $\Gamma$ which acts on a topological space $\tilde{P} \supset P$ that is seen as an orbifold covering or reflection development of $\tilde{P}$.

This means that $P \in \tilde{P}$ is a domain, i.e. its topological boundary in $\tilde{P}$ equals the boundary $\partial P$, and $\Gamma$ is generated by reflections $R_{i}$ in the $(n-1)$ faces $Q_{i} \subset P$ :

- every $R_{i}: \tilde{P} \rightarrow \tilde{P}$ is an involution, $R_{i}^{2}=i d$;

- $R_{i}(P) \cap P=Q_{i}$;

- $R_{i}$ fixes $Q_{i}$.

Regularity. A $\Gamma$-reflection domain $P \subset \tilde{P}$ is called regular, if $\tilde{P}$ is a manifold which admits a smooth Riemannian $\Gamma$-invariant metric. 
Let $2 k_{i j}$ denote the number of $\Gamma$-transformed domains $\gamma(P) \subset \tilde{P}$, including $P=i d(P)$, that contain the $(n-2)$-face $Q_{i} \cap Q_{j} \subset P$ and define $\Gamma$-angles of $P \subset \tilde{P}$ as

$$
\angle_{\Gamma}\left[Q_{i} \cap Q_{j}\right]=\pi / k_{i j}
$$

Notice, that our $P$ and $\tilde{P}$ are not endowed with any metrics so far and the $\Gamma$-angles are purely topological/combinatorial invariants. But if $\tilde{P}$ is a smooth Riemannian manifold and $\Gamma$ acts by isometries, then the $\Gamma$-angles equal the dihedral angles $\angle\left[Q_{i} \pitchfork Q_{j}\right]$.

Example: Rectangular Domains. Let $P$ be a co-simple polyhedral domain $P$, i.e. where the intersections of all $k$-tuples of $(n-1)$-faces satisfy

$$
\operatorname{dim}\left(Q_{1} \cap Q_{2} \cap \ldots \cap Q_{k}\right) \leq n-k+1 .
$$

Then $P$ has a natural regular reflection structure with all dihedral angles $\pi / 2$.

If one glues $P$ with its $R_{i}$-reflected copy along the corresponding face $Q_{i}$, then $P^{\prime}=R_{i}(P) \cup P$ carries again a rectangular reflection structure. Thus, by consecutively applying such reflections with gluing, one constructs a manifold $\tilde{P}$ with the corresponding reflection group $\Gamma$ generated by $R_{i}$ acting on $\tilde{P}$.

For instance, if $P$ is the $n$-cube then $\tilde{P} \subset \mathbb{R}^{n}$ with the reflection group $\Gamma$ being a finite extension of $\mathbb{Z}^{n}$.

\subsection{Gluing around Edges and Dihedral Extremality The- orem.}

Let a compact connected Riemannian mean curvature convex $n$-manifold $P$ with corners be represented by a regular $\Gamma$-reflection domain in $\tilde{P} \supset P$ such that the (geometric) dihedral angles of $P$ are bounded by the corresponding (topological) $\Gamma$-angles,

$$
\angle\left[Q_{i} \pitchfork Q_{j}\right] \leq \angle_{\Gamma}\left[Q_{i} \cap Q_{j}\right] \text { for all }(n-2) \text {-faces } Q_{i} \cap Q_{j} \subset P .
$$

If $\operatorname{scal}(P) \geq 0$, then the manifold $\tilde{P}$ admits a smooth Riemannian $\Gamma$-invariant metric $\tilde{g}_{\text {reg }}$ with positive scalar curvature, scal $\left(\tilde{g}_{\text {reg }}\right)>0$, unless

- all dihedral angles $\angle\left[Q_{i} \pitchfork Q_{j}\right]$ are constant and are equal to $\left\llcorner_{\Gamma}\left[Q_{i} \cap Q_{j}\right]\right.$;

- the mean curvatures of all $(n-1)$-faces $Q_{i} \subset P$ equal zero;

- the scalar curvature of $P$ is everywhere zero.

Proof. Every Riemannian metric $g_{P}$ on $P \subset \tilde{P}$ obviously extends to a unique $\Gamma$-invariant path metric $\tilde{g}$ on $\tilde{P}$ but, typically, this $\tilde{g}$ is singular on the boundary of $P \subset \tilde{P}$.

However, the three inequalities:

$\left[3_{\geq 0}\right] \quad<_{\Gamma}\left[Q_{i} \cap Q_{j}\right]-\angle\left[Q_{i} \pitchfork Q_{j}\right] \geq 0, \operatorname{mn} . \operatorname{curv}\left(Q_{i}\right) \geq 0$ and $\operatorname{scal}\left(g_{P}\right) \geq 0$

say, in effect, that $\operatorname{scal}(\tilde{g}) \geq 0$ in some generalized sense.

In fact, if there is no $(n-2)$-faces at all and $\tilde{P}$ equals the double of $P$ along a mean convex boundary, this $\tilde{g}$ can be easily approximated by a smooth metric $\tilde{g}_{r e g}$ with $\operatorname{scal}\left(\tilde{g}_{r e g}\right)>0$ as is explained in [23] and in [2] for $\operatorname{scal}\left(g_{P}\right)>0$ (also see "Gluing with Positive Scalar Curvature" below) and where non-vanishing of $\operatorname{scal}\left(g_{P}\right)$ at a single point inside $P$ actually suffices because the positivity of scal 
can be "redistributed" over all of $P$ from a single point by a simple perturbation argument. (It is easier to make such metrics with scal $>0$ not on $\tilde{P}$ itself but on $\tilde{P} \times \mathbb{T}^{N}$ by the warping argument from section 12 in $[25$.

This takes the care of rectangular domains, where an essential example is that of $P$ being cubical.

In general, a close look at the "double-gluing/smoothing" argument also shows that it goes well along with $\angle_{\Gamma}\left[Q_{i} \cap Q_{j}\right] \geq \angle\left[Q_{i} \pitchfork Q_{j}\right] \geq 0$ : if $P$ is connected and at least one of the three inequalities $\left[3_{\geq 0}\right]$ is non-strict at some point, then the metric $\tilde{g}$ on $\tilde{P} \supset P$ admits a smooth $\Gamma$-invariant approximation $\tilde{g}_{r e g}$ with $\operatorname{scal}\left(\tilde{g}_{r e g}\right)>0$.

This is obvious for $n=2$ and the general case is not difficult.

Corollary: Dihedral Extremality Theorem. Let $P \subset \mathbb{R}^{n}$ be a convex polyhedron where all dihedral angles are integer fraction of $\pi$, that are $\pi / k$ for some integers $k$.

(An essential instance of this is the $n$-cube $P$ with the dihedral angles equal $\pi / 2$ at all $(n-2)$-faces and where a simple instance of a reflection domain with different angles is the Cartesian product of an (n-2)-cube with a regular triangle where there are $\pi / 2$ and $\pi / 3$ angles.)

Then $P$ is dihedrally extremal: there is no curve-faced domain $P^{\prime} \subset \mathbb{R}^{n}$ combinatorially equivalent to $P$, such that the faces of $P$ have strictly positive mean curvatures and all dihedral angles are bounded by the values of the dihedral angles at the corresponding $(n-2)$-faces of $P$.

Proof Since every Euclidean reflection group $\Gamma$ contains $\mathbb{Z}^{n}$ of finite index, the mean curvature convex dihedrally extremality of these $P$ follows from the Geroch conjecture for the torus $\mathbb{R}^{n} / \mathbb{Z}^{n}$.

Generalizations with scal $\geq 0$. The above does not take much of the Euclidean geometry of $P$, but rather applies to general corned Riemannian manifolds $P$ with $\operatorname{scal}(P) \geq 0$. For instance,

no essential $n$-cubical $P$ with scal $(P) \geq 0$ can have acute dihedral angles and strictly mean curvature convex faces, provided $P$ is spin or $n=\operatorname{dim}(P) \leq 7$.

(The non-spin cases for $n=8,9$ are settled in section 5.3 and [35] allows all n.)

On Irregular Reflection Domains P. An orbifold covering or "reflection development" $\tilde{P}$ of a cornered manifold $P$ may have topological singularities issuing from finite reflection (sub)groups acting at the corners of $P$. These singularities may be avoided if we replace $P$ by its multi-double $P^{\prime \prime}$.... without corners (see section 1.1).

Since $P^{\prime \prime} \ldots$ inherits from $P$ the (strict) inequalities scal $>0$, mean.curv $>0$ and $L_{i j}>\alpha_{i j}$, one can derive lower bounds on $\alpha_{i j}$ whenever the topology of $\tilde{P}^{\prime \prime} \ldots$, allows no metric of positive scalar curvature invariant under the reflection group acting on $\tilde{P}^{\prime \prime} \ldots$.

Yet, this does not help unless $\alpha_{i j}=\pi / k$.

Gluing with Positive Scalar Curvature. Let $X_{1}$ and $X_{2}$ be smooth Riemannian manifolds and let $\gamma: \partial X_{1} \leftrightarrow \partial X_{1}$ be an isometry between their boundaries.

The manifold $X^{1 \cup 2}=X_{1} \cup_{\gamma} X_{2}$ obtained by gluing the two along the boundaries carries a natural continuous Riemannian metric $g^{1 \cup 2}$.

If the shape operators (corresponding to the second fundamental forms) $A_{1}^{*}$ and $A_{2}^{*}$ of the two boundaries "match", i.e. $A_{1}^{*}={ }_{\gamma} A_{2}^{*}$ for $\partial X_{1}$ cooriented 
outward and $\partial X_{2}$ inward, then $g^{1 \cup 2}$ is $C^{1}$-smooth. It follows, that

(*) if the scalar curvatures of $X_{1}$ and $X_{2}$ are strictly positive, then $g^{1 \cup 2}$ can be smoothed to a metric that also has scal $>0$.

Indeed, $g \mapsto \operatorname{scal}(g)$ is a differential operator on Riemannian metrics $g$ on $X^{1 \cup 2}$ that is linear in the second derivatives of $g$ and so the smoothing with any standard smoothing kernel does the job.

The gluing construction from [23] delivers, in effect, a deformation of a metric $g$ on a manifold $X$ with $\operatorname{scal}(X)>0$ and $m n$.curv $(\partial X)>0$ that keeps scal.curv $>0$, that does not change the restriction of $g$ to $\partial X$ and that makes the second fundamental form zero. Then the above gives one a metric with scal $>0$ on the double of $X$.

More generally, let $Y_{1}$ and $Y_{2}$ be, say closed, Riemannian manifolds with metrics $g_{1}$ and $g_{2}$ that are also are "decorated" by quadratic differential forms $A_{1}$ and $A_{2}$ and let us look at compact (complete?) smooth Riemannian manifolds $X=X_{12}=(X, g)$ such that

- the boundary of such an $X$ is decomposed into a disjoint union, $\partial X=$ $\partial_{1} X \sqcup \partial_{2}(X)$, where $\partial X_{1}$ is cooriented by an inward vector field and $\partial_{2}(X)$, by an outward field;

- there are isometries $I_{i}: Y_{i} \rightarrow \partial_{i} X, i=1,2$, that induce the metrics $g_{i}$ from $g$ and send $A_{i}$ to the second quadratic (exterior curvature) forms of $\partial_{i} X \subset X$ where these forms are evaluated with given coorientations.

For instance, $Y_{1}$ and $Y_{2}$ may be be two concentric spheres in $\mathbb{R}^{n}$ with outward coorientations. If $Y_{1}$ is contained in the ball bounded by $Y_{2}$, then these spheres serve as the boundary of the annulus $X$ between them, where, according to our convention, both (second quadratic) forms, $A_{1}$ and $A_{2}$ - on the concave interior $Y_{1}$-boundary with the inward coorientation and on the convex exterior $Y_{2}$-one cooriented by an outward field - are positive definite.

These $X=X_{12}$ may be seen (almost) as morphisms between "decorated" Riemannian manifolds $Y$ : if we glue $X_{23}$ to $X_{12}$ along $Y_{2}$ the resulting metric is $C^{1}$-smooth and the true $X_{13}$ is obtained by smoothing this metric.(One could avoid smoothing if working with $C^{1,1}$-metrics.)

The metrics and quadratic form serve for defining several more interesting smaller categories such as

(1) $\mathcal{B}_{s c>0}^{n}, n=\operatorname{dim}(X)$, the subcategory of the above category where the manifolds $X$ have strictly positive scalar curvatures, $s c(X)>0$, (the category $\mathcal{B}_{s c \geq 0}^{n}$ is equally interesting but slightly harder to handle);

(2) the subcategory $\mathcal{B}_{s c>0}^{n}$ where manifolds $X$ are cobordisms;

(3) the subcategory made by those $X$ where the distance function $x \mapsto$ dist $_{X}\left(x, \partial_{1} X\right)$ is smooth with $\partial_{2} X$ being a constant level of this function.

(These three categories naturally extend to $\infty$-categories with $d$-morphisms being represented by $d$-cubical cornered manifolds, that suggests a "topological field theory" for scal $>0$. Also it is amusing to think of reflection groups as of "enhanced" $\infty$-categories.)

Let us focus our at attention on an, "infinitesimal $\varepsilon$-subcategory" of (3) where the distance between $\partial_{1} X$ and $\partial_{2} X$ equals $\varepsilon \rightarrow 0$ and where the $C^{2}$ distance between the metrics $g_{1}=g_{\mid \partial_{1} X}$ and $g_{2}=g_{\mid \partial_{2} X}$ is also $\leq \varepsilon$ when the two metrics brought to the same manifold, say to $\partial_{1} X$ via the normal projections $\partial_{1} X \leftrightarrow \partial_{2} X$, and answer the following question. 
When can a quadratic form $A_{2}$ on $Y_{2}=\partial X$ be obtained by an equidistant $\varepsilon$-deformation of $Y_{1}=\partial_{1} X$ with a given quadratic form $A_{1}$ on $Y_{1}$..

To effortlessly compute the curvatures, etc. of equidistant deformations $Y_{t}$ of hypersurfaces $Y \subset X$, recall that, in general,

the first derivative of a Riemannian metric $g$ on $X$ restricted to $Y_{t} \subset X$ under the normal equidistant deformation $Y_{t}$ equals the second fundamental form $A_{t}$ of $Y_{t}$, where both forms $g_{t}=g_{\mid Y_{t}}$ and $A_{t}$ are brought to $Y$ by the normal projection $Y \leftrightarrow Y_{t}$,

$$
\frac{d}{d t} g_{t}=A_{t}
$$

while the derivative of the corresponding shape operator $A_{t}^{*}$, that is defined by $g_{t}\left(A_{t}^{*}(\tau), \tau\right)=A_{t}(\tau, \tau)$, for the tangent vectors $\tau \in T\left(Y_{t}\right)$, and then brought to $Y$ by $Y \leftrightarrow Y_{t}$, is expressed by the

Riemannian Hermann Weyl Tube Formuld4:

$\left[\frac{d^{2}}{d t^{2}}\right]$

$$
\frac{d}{d t} A_{t}^{*}=-\left(A_{t}^{*}\right)^{2}+B_{t}
$$

where the operators $B_{t}$ are defined via the sectional curvatures $K$ of $(X, g)$ on the 2-planes $\sigma \subset T_{y}(X), y \in Y_{t}$, that are normal to the tangent spaces $T_{y}\left(Y_{t}\right)$, as follows,

$$
g\left(B_{t}(\tau), \tau\right)=-K(\sigma)
$$

where $\tau$ is a unit vector in the line $\sigma \cap T_{y}\left(Y_{t}\right)$. (We use here the notation from [16], p 43.)

Now, let $U_{\varepsilon}=Y \times[0, \varepsilon]$ for $Y=Y \times 0$, and let $g_{\varepsilon}$ be the following metric on $U_{\varepsilon}$ defined with given smooth metric $g_{0}$ and two smooth quadratic forms $A_{0}$ and $A_{+}$on $Y$,

$$
(++) \quad g_{\varepsilon}(y, t)=g_{0}(y)+t A_{0}(y)+\frac{t^{2}}{2 \varepsilon}\left(A_{+}(y)-A_{0}(y)\right), 0 \leq t \leq \varepsilon .
$$

When $\varepsilon \rightarrow 0$, then

- $g$ the metrics $\left(g_{\varepsilon}\right)_{\mid Y \times t}$ converge to $g_{0}$ in the $C^{2}$-topology; $A_{+}$;

- $A$ the second quadratic forms of $Y=Y \times \varepsilon \subset U_{\varepsilon}$ similarly $C^{2}$-converge to

- scale if trace $\left(A_{+}(y)\right)<\operatorname{trace}\left(A_{0}(y)\right)$ at all $y \in Y$, then $\operatorname{scal}\left(U_{\varepsilon}\right) \rightarrow+\infty$ at all $x \in U_{\varepsilon}$

In other words, an infinitesimal positive scalar curvature cobordism/morphism can transform a quadratic form $A_{0}$ to a given $A_{+}$, whenever the mean curvature (trace) of the latter is strictly smaller than that of the former.

(It is helpful to visualize this by thinking of $Y_{\varepsilon}=Y \times \varepsilon$ as the equidistant $\varepsilon$-deformation of an equatorial $(n-1)$-subsphere $Y_{0}$ in the $n$-sphere of radius $r$, with $U_{\varepsilon}$ being the annulus pinched between these two spheres, where $\operatorname{scal}\left(U_{\varepsilon}\right)=$ $n(n-1) / r^{2}$ and the second fundamental form $A_{0}$ of $Y_{0}$ is zero.

\footnotetext{
${ }^{4}$ This is a most useful formula in Riemannian geometry that directly leads to geometrically significant results, e.g. to basic comparison theorems (see [16]), unlike still persistent roundabout computations with curvature tensors and Jacobi fields used for this purpose.
} 
A relevant example here is where $r \rightarrow 0$ and $\varepsilon=$ const $\cdot r^{2}$ and where the second fundamental form $A_{+}$of $Y_{\varepsilon}$ is, negative, being, roughly, -const $\cdot \mathbf{1}$.)

Proof. The claim $\bullet_{g}$ is obvious, $\bullet_{A}$ follows from $\left[\frac{d}{d t}\right]$, while the asymptotic estimate $\operatorname{scal}\left(U_{\varepsilon}\right) \sim \operatorname{const} \cdot \varepsilon^{-1}\left(\operatorname{trace}\left(A_{0}\right)-\operatorname{trace}\left(A_{+}\right)\right)$is seen with $\left[\frac{d^{2}}{d t^{2}}\right],[K]$ and Gauss' theorema egregium.

Gluing Corollary. Let $X_{1}$ and $X_{2}$ be manifolds with strictly positive scalar curvatures and let $\gamma: \partial X_{1} \leftrightarrow \partial X_{2}$ be an isometry.

If the mean curvatures of the two boundaries satisfy

$$
\text { mn.curv }{ }_{\partial X_{1}}\left(y_{1}\right)+m n . \operatorname{curv}_{\partial X_{2}}\left(y_{2}\right) \geq 0 \text { for all } y_{1} \in \partial X_{1} \text { and } y_{2}=\gamma\left(y_{1}\right) \in \partial X_{2} \text {, }
$$

then the metric $g^{1 \cup 2}$ on the manifold $X^{1 \cup 2}$ obtained by gluing the two along their boundaries can be perturbed in a neighbourhood of the glued boundaries to a metric of positive scalar curvature on $X^{1 \cup 2}$.

Proof. If we apply $(++)$ to $g_{0}^{\prime}(y)=g_{0}(y)+\delta(y)$ instead of $g_{0}(y)$ for $\delta(y)=$ $g_{0}(y)-g_{\varepsilon}(y, \varepsilon)$ we end up with $g_{\varepsilon}^{\prime}(y, \varepsilon)=g_{0}(y)$. Thus, an arbitrary small $C^{2}-$ perturbation of $g_{0}$ allows us to achieve $g_{\varepsilon}(y, \varepsilon)=g_{0}$.

Now we can modify the metric on one of the two manifolds, say on $X_{1}$, such that

- the modified metric equals the original one away from an arbitrary small neighbourhood (that is our $U_{\varepsilon} \supset Y=\partial X_{1}$ ) of the boundary of $X_{1}$;

- the restriction of the modified metric to the boundary remains equal the original metric (corresponding to the above $g_{0}$ );

- the modified metric has scal >0;

- the second fundamental form of the boundary with respect to the modified metric has the second fundamental form opposite to that of the boundary of $X_{2}$.

Then, by the above $(*)$, the manifold $X_{1}$ with the modified metric can be glued to $X_{2}$ and the proof follows.

Gluing with scal $>\kappa \neq 0$. The above equally applies to manifolds with scalar curvatures bounded from below by any constant $\kappa$, not necessarily $\kappa=0$ :

If manifolds $X_{1}$ and $X_{2}$ with their scalar curvatures bounded from below by a constant $\kappa$ are glued by an isometry $\gamma$ between their boundaries the mean curvatures of which satisfy the above positivity $(\geq 0)$ of their sum inequality, then the metric $g^{1 \cup 2}$ on the resulting manifold $X^{1 \cup 2}=X_{1} \cup_{\gamma} X_{2}$ can be approximated by smooth metrics $g_{a p p}$ on $X^{1 \cup 2}$ with $\operatorname{scal}\left(g_{a p p}\right)>\kappa$ where these metrics $g_{a p p}$ can be chosen equal $g^{1 \cup 2}$ away from an arbitrarily small neighborhood of the glued boundaries $\partial X_{1} \underset{\gamma}{=} \partial X_{2} \subset X^{1 \cup 2}$.

Remarks. (a) The above style local "gluing + smoothing" appears in different forms in [23, 2, 39] and non-local smoothing with the Ricci flow is suggested in 37 .

(b) Probably, ideas from [39, 37, 31] and [14 may help to establish a version of this under the non-strict assumption $\operatorname{scal}\left(X_{i}\right) \geq \kappa, i=1,2$, and with the corresponding non-strict conclusion $\operatorname{scal}\left(g_{a p p}\right) \geq \kappa$ for most (all) manifolds $X_{1}$ and $X_{2}$.

(c) If two given smooth Riemannian metrics on $Y_{1}$ and on $Y_{2}=Y_{1}$ can be included in a continuous family of metrics with positive scalar curvatures, 
then, by combining the above with 24, one sees that the existence of a noninfinitesimal cobordism as in the above (3) with given second quadratic forms $A_{1}$ on $Y_{1}$ and $A_{2}$ on $Y_{1}$ and $Y_{2}$ does not need the assumption $\operatorname{trace}\left(A_{1}\right)>\operatorname{trace}\left(A_{2}\right)$. Let us spell it out in detail.

Let $A_{1}$ and $A_{2}$ be smooth quadratic forms on a manifold $Y$ an let $g$ be a metric on $X=Y \times[1,2]$, such that

$(+)$ the metrics $g_{\mid Y \times t}$ on $Y \times t=Y$ have strictly positive scalar curvatures for all $t \in[1,2]$.

Then there exists a homotopy $g_{\tau}$ of the metric $g=g_{\tau=0}$ on $X$, such that

- the metrics $g_{\tau \mid Y \times t}$ on $Y=Y \times t$ have positive scalar curvatures for all $t \in[1,2]$ and $\tau \in[0,1]$

- the result of this homotopy - the metric $g_{\tau=1}$ on $X$ has strictly positive scalar curvature;

- the homotopy is constant on $Y \times 1$ and $Y \times 2$,

$$
g_{\tau \mid Y \times 1}=g_{\mid Y \times 1} \text { and } g_{\tau \mid Y \times 2}=g_{\mid Y \times 2} \text { for all } \tau \in[0,1] ;
$$

- the second quadratic forms of $Y \times 1$ and $Y \times 2$ in $\left(X, g_{\tau=1}\right)$ equal $A_{1}$ and $A_{2}$ correspondingly.

- the submanifolds $Y \times t \subset X$ are equidistant to $Y \times 1$ as well as to $Y \times 2$ for all $t \in[1,2]$ (as in the above (3)) with respect to $g_{\tau=1}$; moreover, if $g$ has this equidistance property, then one can have all $g_{\tau}$ with this property as well.

Question. When does a closed subset $Z$ in a Riemannian manifold $X$ equal the intersection of an decreasing sequence of smooth domains $U_{i} \subset X$ where the induced metric $g_{i}$ on the boundaries $Y_{i}=\partial U_{i}$ have $\operatorname{scal}\left(g_{i}\right) \rightarrow+\infty$ for $i \rightarrow \infty$ ?

Is there a sufficient condition representable by an inequality $\operatorname{dim}_{?}(Z)<$ $\operatorname{dim}(X)-2$ for some notion of dimension as it is the case for piecewise smooth polyhedral subsets $Z \subset X$ of codimension $>2$ by the argument from 24].

A related question (we reiterate it in section 3 ) is that of finding "nice" functions $\phi_{i}$ on $X \backslash Z$ that blow up at $Z$ and such that the intersection of certain $\phi_{i}$-bubbles equals $Z$.

(d) There are global PDE constructions of metric with positive scalar curvatures on "glued manifolds" like the above $X^{1 \cup 2}$ under integral rather than point-wise assumptions on the mean curvatures (e.g. see [11] and references therein) but the available results of this kind apply so far only to rather special metrics.

\subsection{Dihedral Rigidity Conjecture.}

The above does not say what are dihedrally extremal mean curvature domains and, more generally, what are the above cornered Riemannian $n$-manifold $P$ where

$$
\angle\left[Q_{i} \pitchfork Q_{j}\right]=\angle_{\Gamma}\left[Q_{i} \cap Q_{j}\right], \text { mn.curv }\left(Q_{i}\right)=0 \text { and } \operatorname{scal}\left(g_{P}\right)=0 .
$$

Probably, they are all isometric to convex Euclidean polyhedra. In particular,

bounded Euclidean polyhedral reflection domains $P \subset \mathbb{R}^{n}$ are, conjecturally, dihedrally rigid. 
Namely,

let a curve-faced $P^{\prime} \subset \mathbb{R}^{n}$ have not necessarily strictly positive mean curvatures of all their faces $\geq 0$ and all dihedral angles bounded by the corresponding angles of $P$.

Then, conjecturally, all faces of $P^{\prime}$ are flat; moreover, $P^{\prime}$ is obtained from $P$ by parallel translations of its faces followed by an isometry.

Five Incomplete Proofs. (1) The most transparent case of the problem is where $P$ is a curve-faced cubical polyhedron in the Euclidean 3 -space $\mathbb{R}^{3}$.

If $P$ is extremal, then all 2 -faces of it are minimal surfaces meeting each other at the angles $\pi / 2$. If such a face $Q$ can be slightly moved inside $P$ with a strict decrease of its area, then it can be perturbed to strict mean convexity and if every such a move strictly increase the area of $Q$, then one could make $Q$ strictly mean curvature concave by such a move.

Thus, we may assume that

$Q$ includes in a continuous family $Q_{t} \subset P$ of minimal faces normal to the rest of the boundary of $P$. Now, following Schoen-Yau 45, (compare 8]) we observe that the second variation integral for area $(Q)$ equals the integral of the Gauss curvature of $Q$ plus the boundary term that is the integral over the curve $\partial Q$ of the difference between the mean curvatures of $\partial Q$ in $Q$ and of the surface of $P$ normal to $Q$.

Thus, the (non-strict) positivity of the second variation implies that the integral of the Gauss curvature of $Q$ plus the integral of the curvatures of the four edges of its boundary is non-negative. On the other hand, the four vertices contribute $2 \pi$ to the Gauss-Bonnet integral; hence, $Q$ must be flat.

The only unsettled point in this proof is hating that nothing bad happens at the corners of $P$ but this does not seem to be difficult. In fact, the GaussBonnet prism inequality (see 5.4) does work in this case and implies rigidity of all, not necessarily (reflection) convex Euclidean prisms.

If $P$ is a curve-faced cubical polyhedron in $\mathbb{R}^{n}$ for $n \geq 4$, one may apply the Schoen-Yau dimension reduction argument [46] or rather the warped product version of it from 25, where, one needs doing it only once, and where the dimension restriction $n \leq 6$ is unnecessary since all we need after all is a certain perturbation of a smooth face $Q$.

(2) Let us turn now to general reflection domains and recall that if $X$ is a compact manifold with zero scalar curvature $g_{0}$, then, according to [28, $g_{0}$ can be perturbed to $g_{1}$ with $\operatorname{scal}\left(g_{1}\right)>0$ unless $g$ is Ricci flat. Probably, a similar perturbation is possible for the above cornered Riemannian $n$-manifold $P$ with

$$
\angle\left[Q_{i} \pitchfork Q_{j}\right]=\angle_{\Gamma}\left[Q_{i} \cap Q_{j}\right], \text { mn.curv }\left(Q_{i}\right)=0 \text { and } \operatorname{scal}\left(g_{P}\right)=0 .
$$

This suggest the following

Question. Let $P \subset \mathbb{R}^{n}$ be a preconvex Plateau-hedron where at least one face is non-flat. When does $P$ admit a perturbation to a strictly mean curvature convex polyhedron $P^{\prime}$ with all dihedral angles $<_{i j}\left(P^{\prime}\right) \leq<_{i j}(P)$ ? (A general positive answer would settle the rigidity problem.)

Example. Let $P \subset \mathbb{R}^{3}$ be bounded by a catenoid and a pair of hyperplanes normal to its axes. (This $P$ has depth two as it has no corners.) It seems easy to decide if $P$ can be perturbed to a strictly mean convex polyhedron with a decrease of its two (circular) dihedral angles. 
(3) Let $\tilde{P}$ be the above manifold where reflection group $\Gamma$ acts with $P \subset \tilde{P}$ being a $\Gamma$-reflection domain and let $\tilde{g}$ on $\tilde{P} \supset P$ be the continuous metric coming from $P$.

The above gluing argument says in this case that $\tilde{g}$ admits a smooth $\Gamma$ invariant approximation by metrics $\tilde{g}_{r e g}$ with $\operatorname{scal}\left(\tilde{g}_{r e g}\right)>-\varepsilon$ for arbitrarily small $\varepsilon>0$. Then the positive solution to the $C^{0}$-rigidity problem $\bullet_{2}$ in 1.9 would show that the metric $\tilde{g}$ is flat.

(4) Torus Conjecture. Let $\tilde{X}$ be the universal covering of the $n$-torus $X$ with a smooth non-flat Riemannian metric.

Then, conjecturally, $\tilde{X}$ can be exhausted by cubical strictly mean curvature convex cornered domains $\tilde{P} \subset \tilde{X}$ with all dihedral angles bounded by $\pi / 2$.

Half-Proof. Such domains $\tilde{P}_{k}$ with singular faces are constructed as follows.

Let $X^{\sim k}=X / k \mathbb{Z}^{n}$ for $Z^{n}=\pi_{1}(X)$ and let $Y_{1} \subset X^{\sim_{k}}$ be a minimal hypersurface homologous to an $(n-1)$-subtorus in $X^{\sim_{k}}$. Let $X_{1}^{\sim_{k}}$ be (possibly singular for $n \geq 8$ ) space obtained by cutting $X^{\sim k}$ along $Y_{1}$ where the boundary of $X_{1}^{\sim k}$, denoted $\partial X^{\sim_{k}}=Y_{ \pm 1}$, consists of two copies of $Y_{1}$.

Take an $(n-1)$-volume minimizing hypersurface $Y_{2} \subset X_{1}^{\sim k}$ with $\partial Y_{2} \subset \partial X_{1}^{\sim k}=$ $Y_{ \pm 1}$ that represent the relative homology class "suspending" the class of some " $(n-2)$-subtorus" in $Y_{1}$.

Cut $X_{1}^{\sim_{k}}$ along $Y_{2}$ and take the result in space for $X_{2}^{\sim_{k}}$. This $X_{2}^{\sim_{k}}$ is a cornered 2-cubical space where the boundary $\partial X_{2}^{\sim_{k}}$ consists of two pairs mutually orthogonal faces that are $Y_{ \pm 1}^{c u t}$ and $Y_{ \pm 2}$.

Cut $X_{2}^{\sim_{k}}$ along a a volume minimizing hypersurface $Y_{3}$ in $\partial X_{2}^{\sim_{k}}$ with boundary in $\partial X_{2}^{\sim_{k}}$ and thus obtain a 3 -cubical $X_{2}^{\sim_{k}}$ with norma $\mathrm{n}$ dihedral angles where these are defined.

Keep doing this unless you arrive a singular mean curvature $n$-cubical cornered space $X_{n}^{\sim_{k}}$ with normal faces that lifts to a singular cubical domain $\tilde{P}_{k} \tilde{X}$, where $\tilde{X}$ can be exhausted by such $\tilde{P}_{k}$ for $k \rightarrow \infty$.

What remains is to smooth the singularities in these $\tilde{P}_{k}$. Prior to smoothing one has to modify the construction by taking $\varepsilon$-bubbles with small $\varepsilon>0$ (defined below) instead of minimal hypersurfaces $Y_{i} \subset X_{i-1}^{\sim k}$ (corresponding to $\varepsilon=0$ ).

Such bubbles do exist if $X$ is non-flat (this seems obvious but a proof will not hurt) and then the resulting polyhedra $\tilde{P}_{k} \subset \tilde{X}$ are strictly mean curvature positive. Probably - and this is what we do not prove - such polyhedral domains $\tilde{P}$ can be always approximated by face-wise smooth strictly mean curvature convex domains with $\pi / 2$ dihedral angles .

A construction of such strictly mean curvature convex cubical domains $Q$ in manifolds $\tilde{X}$ with a piecewise smooth metrics $\tilde{g}$ would imply dihedral rigidity of reflection domains, since one could construct a metric of positive scalar curvature on $X$ (or rather on some $X^{\prime}$ admitting a degree 1 map to the $n$-torus) by "gluing $Q$ around the corners".

(5) Probably, one can make sense of the Dirac $D_{\tilde{g}}$ operator being (nonstrictly) positive on $(\tilde{P})$, confront this with existence of harmonic spinors twisted with flat bundles as in 23], and use a piecewise smooth version of Bourguignon theorem on parallel spinors.

Topological Mean Convex Exhaustion Problem. Let $\mathcal{P}$ be a combinatorial class of (potential) cornered domains $P$ with numbers $\alpha_{i}$ attached to the edges of $P \in \mathcal{P}$. 
Let $\tilde{X}$ be a smooth manifold acted upon by a discrete cocompact group $\Gamma$. For instance, $\tilde{X}$ may be a universal covering of a closed manifold $X$ with $\pi_{1}(X)=\Gamma$.

If $\tilde{X}$ is endowed with a smooth $\Gamma$-invariant RIemannian metrics $\tilde{g}$, we say that $\tilde{X}=(\tilde{X}, \tilde{g}): \Gamma$ is exhaustively dominated by $\left(\mathcal{P}, \alpha_{i}\right)$ if $\tilde{X}$ can be exhausted by mean curvature convex domains $\tilde{P} \in \mathcal{P}$ where all dihedral angles in all $P$ are bounded by $\alpha_{i}$. (The definition of combinatorial equivalence allows maps $\tilde{P} \rightarrow P \in \mathcal{P}$ of "positive degrees" where, possibly, $\operatorname{dim}(\tilde{P})>\operatorname{dim}(P)$.)

Say that $\tilde{X}: \Gamma$ is topologically exhaustively dominated by $\left(\mathcal{P}, \alpha_{i}\right)$ if $(\tilde{X}, \tilde{g})$ is exhaustively dominated by $\left(\mathcal{P}, \alpha_{i}\right)$ for all smooth $\Gamma$-invariant RIemannian metrics $\tilde{g}$ on $\tilde{X}$.

For instance, the torus conjecture claims that the topological $n$-torus $T^{n}=R^{n} / \mathbb{Z}^{n}$ is so dominated by the (combinatorial class of the) cube $\left(\square^{n}, \alpha_{i}=\pi / 2\right)$.

In general, given $\tilde{X}$ acted upon by $\Gamma$ and a combinatorial class $\mathcal{P}$ with the edge set $I=\{i\}$, we denote by $A_{\mathcal{P}}(\tilde{X}: \Gamma) \subset \mathbb{R}^{I}$ the set of vectors $\left\{\alpha_{i}\right\} \in \mathbb{R}^{I}$ for which $\left(\mathcal{P}, \alpha_{i}\right)$ topologically exhaustively dominate $\tilde{X}: \Gamma$.

This set $A_{\mathcal{P}}$ is a topological invariant of $(\tilde{X}: \Gamma)$ (or equivalently of the quotient manifold $\tilde{X} / \Gamma$ for free actions) and the problem is to evaluate it in particular cases.

Examples. (i) Let $P \subset \mathbb{R}^{m}$ be a convex polyhedron with dihedral angles $\alpha_{i}$.

Let $X$ be a closed smooth $n$-manifold that admits a continuous map $X \rightarrow \mathbb{T}^{m}$ of "non-zero degree", i.e. such that the fundamental cohomology class of the torus goes to a non-zero class in $H^{m}(X, \mathbb{Q})$ and let $\tilde{X}$ the Galois $\mathbb{Z}^{m}$-covering of $X$ induced by the universal covering $\mathbb{R}^{m} \rightarrow \mathbb{T}^{m}$.

Is $\tilde{X}: \Gamma$ topologically exhaustively dominated by $\left(\mathcal{P}, \alpha_{i}\right)$ for the combinatorial class $\mathcal{P}$ of this $P \subset \mathbb{R}^{m}$ ?

(ii) Does this remain so, for $\Gamma \neq \mathbb{Z}^{m}$, if $\tilde{X}$ admits a smooth proper distance decreasing map $\tilde{X} \rightarrow \mathbb{R}^{n}$ of non-zero degree?

Here "non-zero degree" means that the pullback of a generic point is $\mathbb{Q}$-nonhomologous to zero in $\tilde{X}$ and "distance decreasing" is understood relative to some $\Gamma$-invariant metric in $\tilde{X}$.

(iii) Are the universal coverings $\tilde{X}$ of manifolds $X$ with infinite $K$-area (defined in [19]) topologically exhaustively dominated by these $\left(\mathcal{P}, \alpha_{i}\right)$ ?

(iv) Let the hyperbolic $n$-space $H^{n}$ of constant curvature -1 be exhausted by curve-faced polyhedral domains $P$ of a certain combinatorial type $\mathcal{P}$ with umbilical faces with positive mean curvatures and acute dihedral angles.

Is then $H^{n}: \Gamma$ topologically exhaustively dominated by $\left(\mathcal{P}, \alpha_{i}=\pi / 2\right)$ ?

Does a similar property hold true for exhaustions of $H^{n}$ by domains $P_{1} \subset$ $P_{2} \subset \ldots . \subset H^{n}$ of variable combinatorial types?

What are combinatorial classes $\mathcal{P}$, such that $\left(\mathcal{P}, \alpha_{i}=\pi / 2\right)$ topologically exhaustively dominate (the universal coverings of) $\mathbb{Q}$-essential closed manifolds $X$ with other "large" fundamental groups $\Gamma$ ?

(Recall that "Q্Q-essential" means that the fundamental cohomology class $[X]_{\mathbb{Q}} \in H^{n}(X ; \mathbb{Q}), n=\operatorname{dim} X$, comes from the cohomology of $\Gamma$.)

Particular instances of interesting "large" groups are cocompact lattices in Lie Groups and Cartesian projects of world hyperbolic groups.

Probably, there are significantly of more such $\mathcal{P}$ for hyperbolic groups than for products of these and than for groups co-compactly acting on non-hyperbolic symmetric spaces. 
Let is limit the $\Gamma$ invariant metrics $\tilde{g}$ on $\tilde{X}$ to those where $\operatorname{scal}(\tilde{G}) \geq-1$. Then, besides bounds on the dihedral angles of domains $P_{k}$ exhausting $\tilde{X}$ by given numbers $\alpha_{i}$ one may requite lower bounds on the mean curvatures of the faces of these domains.

What are realizable (by some exhaustions) possibilities for such bounds if, for instance,

(•) $\tilde{X}$ is symmetric space with non-positive curvature;

(•) $\tilde{X}$ is acted upon by an isometry group $\Gamma$ (for a metric $\tilde{g}$ with $\operatorname{scal}(\tilde{g}) \geq-1)$ and it admits a proper equivariant map of non-zero degree onto a symmetric space that is isometrically and co-compactly acted upon by $\Gamma$.

(*) What are extremal/rigid corned domains in spaces of constant (positive or negative) curvatures with given bounds on the dihedral angles and lower bounds on the mean curvatures of their faces?

For instance, let $P \subset S^{n} \subset \mathbb{R}^{n+1}$ be a reflection domain, e.g. the spherical simplex $\Delta$ with all dihedral angles equal $\pi / 2$ and let $P$ be a mean convex cornered manifold with all dihedral angles $\leq \pi / 2$ that admits a 1-Lipschitz combinatorial equivalence $f: P \rightarrow \Delta$.

Then, if $P$ is spin, the above argument combined with Llarull's theorem 34 shows that

there is a point $p \in P$, such that $\operatorname{scal}_{p}(P) \leq \operatorname{scal}_{f(p)}\left(S^{n}\right)$.

But it is unclear if the equality $\operatorname{scal}_{p}(P)=\operatorname{scal}_{f(p)}\left(S^{n}\right)$ at all $p \in P$ implies that $f$ is an isometry.

\subsection{Billiards, Pure Edges and Ramified Coverings.}

Most (if not all) of our understanding of mean curvature convex cornered manifolds $P$ is derived from the geometry of minimal varieties and/or the Dirac operator on orbifold coverings (reflection developments) $\tilde{P}$ of $P$.

For example if $P \subset X=X^{n}$ is a $n$-cubical polyhedral domain, then the quotient $P_{\circ}=\tilde{P} / \Gamma_{\circ}$, for a subgroup $\Gamma_{\circ}=\mathbb{Z}^{n} \subset \Gamma$ of finite index in the corresponding reflection group, $\Gamma$ acting on $\tilde{P}$, is a $\mathbb{T}^{n}$-essential manifold, i.e. it comes with a map of positive degree $P_{\circ} \rightarrow \mathbb{T}^{n}$.

The relevant minimal subvarieties in $P_{\circ}$ are those representing the $(n-1)$ homology classes that come as pullbacks from $(n-1)$ subtori in $\mathbb{T}^{n}$.

There are infinitely many of these classes, but only $n$ of them, the ones that correspond to the coordinate subtori, have a simple representation in $P$, namely by (eventually minimal) hypersurfaces separating pairs of opposite faces.

The remaining ones are similar to multiply reflected periodic orbits of billiards in polygonal domains.

Question. Is there a counterpart of these multiply reflective minimal hypersurfaces for polyhedral domains $P$ that are not reflection domains?

A similar issue arises for the Dirac operator.

How can one descent Dirac operator $D$ proofs of non-existence of metrics with $s c l>0$ from $\tilde{P}$ to $P$ ?

One problem is the discontinuity of $D$ for the natural (only continuous but not smooth) extension of the Riemannian metric from $P$ to $\tilde{P} \supset P$.

Even more serious difficulty stems from the fact that it is not $D$ itself is used but $D$ twisted with (almost flat) vector bundles $V$ over $\tilde{P}$, where these $V$ is bu 
no means $\Gamma$-invariant.

An essential difference between ordinary billiards and what we have here is that the dynamics and geometry of billiards are shaped by interactions of the orbits with the faces of $P$ while the geometries of minimal hypersurfaces and of Dirac crucially depend on what happens at the edges of $P$.

Below is an attempt to isolate the edge geometry.

Pure Edges without Faces. Let $X=X^{n}$ be a closed manifold and $Z=Z^{n-2} \subset$ $X$ a closed submanifold of codimension 2, e.g. a knot in the 3 -sphere.

Consider all metrics on $X$ that are smooth with non-negative scalar curvatures away from $Z$ and such that the geometry near $Z$ is corner singular with angle $\alpha$, i.e. a neighbourhood of $Z$ in $X$ is isometric to $\left(Z, g_{Z}\right) \times C_{\alpha}$ where $g_{Z}$ is a smooth Riemannian metric on $Z$ and $C_{\alpha}$ is a surface with a rotationally symmetric Riemannian metric that is singular at a single point $c_{0} \in C_{\alpha}$ where its tangent cone has total angle $\alpha$. For example, if $\alpha=2 \pi$ then $C_{\alpha}$ is non-singular.

Denote by $\alpha_{\min }(X, Z)$ the infimum of these angles of all above metrics.

This is a topological invariant of the pair that can be bounded from below by looking at the ramified coverings of $X$. Namely, if there is such a covering $\tilde{X}$ with ramification of order $\leq k$ that admits no smooth metric with positive scalar curvature then, clearly, $\alpha_{\min }(X, Z) \geq 2 \pi / k$.

Question. Are there pairs $(X, Z)$ where $\alpha_{\min }(X, Z)$ is finite but yet not of the kind $2 \pi / k$ for any integer $k$ ?

The simplest instance of where such bounds are available is where $X=S^{2} \times S^{1}$ and where $Z$ equals a union of "coordinate circles", i.e. $X=Z_{0} \times S^{1}$ for a finite subset $Z_{0}$ in the sphere $S^{2}$ and where the geometries near these circles $Z_{i}=z_{i} \times S^{1} \subset X=S^{2} \times S^{1}, z_{i} \in Z_{0}$, are corner singular with (not necessarily mutually equal) angles $\alpha_{i}$.

On can show, as we do it in section 5.4 that, albeit singular, $X$ contains a minimal surface $Y$ in the homology class of the 2-sphere $S^{2} \times z_{0} \subset X$ to which the Gauss-Bonnet theorem applies and yields the (sharp!) inequality

$$
\sum_{i}\left(2 \pi-\alpha_{i}\right) \leq 4 \pi .
$$

Remark. Some geometry questions on general cornered manifolds $P$ with $\operatorname{scal}(P)>0$ reduce to those about the above "pure edged" spaces with the multi-doubling procedure from section 1.1.

Problem. What are complete singular Riemannian spaces that are locally isometric to Cartesian products of flat manifolds and 2-dimensional Riemannian cones?

Does, for instance, every stably parallelizable manifold admit such a singular Riemannian metric?

\section{$3 \mu$-Area and $\mu$-Bubbles.}

The variational approach indicated in section 1.4 for construction of Plateauhedra also applies to more general curve-faced polyhedra with prescribed mean curvatures of the faces and given dihedral angles at the edges as follows. 
An open or closed subset $U \subset X$ is a domain if its boundary also serves as the topological boundary of the complement to the closure of $U$, that is

$$
\partial U=\partial(\operatorname{int}(U))=\partial(\operatorname{clos}(U))=\partial(X \backslash U)=\partial(X \backslash \operatorname{clos}(U))=\partial(X \backslash \operatorname{int}(U)) .
$$

Each component $Y$ of the boundary of a domain $U$ admits two coorientations represent by arrows directed toward interior or exterior of $U$. Accordingly, we denote by $[\ldots \stackrel{\text { in }}{\leftarrow} Y] \subset X$ the germ of the intersection of $U$ with an arbitrarily small neigbourhood $W_{\varepsilon}$ of $Y$ in $X$, where we agree that $Y \subset[\ldots \stackrel{\text { in }}{\leftrightarrow} Y] \subset X$ and we denote by $[Y \stackrel{\text { out }}{\leftarrow} \ldots]$ such a germ of the exterior of $Y$ that is the complement of the interior of $U$ in $W_{\varepsilon}$.

Observe that this notation makes sense even if a hypresurface $Y$ does not bound anything in $X$, but only divide its small neighbourhood into two parts, one regarded as interior and the other as exterior of $Y$.

A Borel measure $\mu$ on $X$ defines a closed 1-cochain on cooriented hypersurfaces $Y$ written is $\mu[\ldots \stackrel{\text { in }}{\leftrightarrow} Y]$, where the function $Y \mapsto \mu[\ldots \stackrel{\text { in }}{\leftrightarrow} Y]$ changes by an additive constant under a change of the representative of the germ $[\ldots \stackrel{\text { in }}{\leftarrow} Y] \subset X$.

If $X$ is also endowed with a Riemannian metric we define the $\mu$-area of $Y$ by

$$
\operatorname{area}_{\mu}(Y)=\operatorname{def}_{\operatorname{vol}_{n-1}}(Y)-\mu[\ldots \stackrel{\text { in }}{\leftarrow} Y]
$$

where $\operatorname{vol}_{n-1}$ stands for the $(n-1)$-dimensional Hausdorff measure.

Call a hypersurface $Y \subset X$ a $\mu$-bubble if it locally minimizes the function $Y \mapsto \operatorname{area}_{\mu}(Y)$.

If $\mu$ is given by a continuous density function $\phi(x), x \in X$, i.e. $\mu=\phi d x$, then the mean curvature of a $\mu$-bubble $Y \subset X$, obviously, satisfies mn.curv $(y)=\phi(y)$. These $\mu$-bubbles are also called $\phi$-bubbles.

In particular, $C$-bubbles with constant mean curvature equal $C$ corresponds to $\mu$ being proportional to the Riemannian $n$-volume.

Example. If $X=\mathbb{R}^{n}, n \geq 3$, and $\phi(x)=(n-1)\|x\|^{-1}$, then the $R$-spheres defined by $\|x\|=R$ are (non-strictly) locally minimizing $\phi$-bubbles.

Questions. Can a (stable?) minimal submanifold $Z$ of codimension $\geq 2$ in a Riemannian $n$-manifoldc $X$ be "surrounded" by $C$-bubbles (with an arbitrarily large positive constant $C$ ) that are small perturbations of the levels of the function $(n-1) \operatorname{dist}(x, Z)^{-2}$ ? (This, possibly, can done by arranging suitable traps for such bubbles, see section 4.2.)

When, in general, does a closed subset $Z \subset X$ admit arbitrarily small neighbourhoods $U \supset Z$ with (smooth?) boundaries $\partial U$ of (almost?) constant mean curvatures?

For instance, when can a subset $Z \subset X$ be surrounded by $\phi$-bubbles that approximate levels of a function $\phi \sim(n-1) \operatorname{dist}(x, Z)^{-2}$ ?

Is this possible for smooth non-minimal submanifolds and/or for singular minimal subvarieties $Z \subset X$ with $\operatorname{codim}(Z) \geq 2$ ?

What happens in this regard to piecewise smooth subpolyhedra and to real algebraic subsets $Z$ ?

Is there anything of this kind for nice(?), possibly fractal, subsets $Z \subset X$, e.g. for singular loci of minimal subvarieties in $X$ ?

Do constructions of minimal and mean curvature convex hypersurfaces from 22 extend to $\phi$-bubbles in non-complete manifolds such as $X \backslash Z$ with suitable functions $\phi(x)$ that have "pole-like singularities" on $Z$ ? 
If $X$ is a manifold with a smooth boundary $\partial X$ and $\mu=\mu_{\partial}$ is given by a continuous density function on $\partial X$, say by $\psi_{\partial}\left(x^{\prime}\right), x^{\prime} \in \partial X$, then $\mu_{\partial}$-bubbles $Y \subset X$ with $\partial Y \subset \partial X$ meet $\partial X$ at the (dihedral) angle $\angle=\angle(Y \pitchfork \partial X)$ that, obviously, satisfies $\cos \angle\left(y^{\prime}\right)=\psi_{\partial}\left(y^{\prime}\right)$ for all $y^{\prime} \in Y \cap \partial X$. Moreover, this equality remans true for the $\mu_{\partial}^{\prime}$-bubbles where $\mu^{\prime}=\mu_{\partial}+\phi \cdot v_{\text {ool }}$ for $\phi=\phi(x)$ being an arbitrary continuous function on $X$.

Question. What is the minimal regularity of a measure $\mu$ needed for the existence of $\mu$-bubbles and their regularity comparable to that of minimal hypersurfaces? Does the condition $\mu \leq$ const $\cdot$ Hau $_{n-1}$ for the $(n-1)$-Hausdorff measure $H_{a u_{n-1}}$ suffice?

Remarks. (a) If $X$ has trivial $(n-1)$-homology, $H_{n-1}(X)=0$, then minimal $\varepsilon$-bubbles are associated to the supporting lines $a-\varepsilon v=$ const of the (convex hull of the) isoperimetric profile of $X$ in the positive quadrant of the $(a, v)$-plane, where profile $_{i s o p}(X) \subset \mathbb{R}_{++}^{2}$ is defined as the image of the map from the space of compact domains $P$ to the plane given by

$$
P \mapsto\left(a=\operatorname{vol}_{n-1}(\partial P), v=\operatorname{vol}_{n}(P)\right),
$$

where, observe, the boundary of $\operatorname{profile}_{i s o p}(X)$ is contained in the critical set (curve) of this map.

(If $X$ is a Galois coverings of compact manifold, one usually works with the Foelner-Vershik profile that is the convex hull of the logarithmic map $P \mapsto$ $\left(\log \operatorname{vol}_{n-1}(\partial P), \log \operatorname{vol}_{n}(P)\right)$.)

(b) $\mu$-Bubbles are well defined for closed hypersurfaces $Y$ which bound (noncompact) domains with infinite $\mu$-measures, like $Y \times 0 \subset X=Y \times \mathbb{R}$, provided the $\mu$-measures of the regions between $Y$ and hypersurfaces $Y^{\prime}$ homologous to $Y$ are finite.

\subsection{Poly-Bubble-Hedra.}

A Riemannian $n$-manifold $P$ with corners, e.g. a polyhedral domain in an ambient Riemannian manifold $X=X^{n}$, is called a $P B$-hedron if all its $(n-1)$ faces $Q_{i}$ have constant (possibly mutually non-equal) mean curvatures $m_{i}$ and the dihedral angles $\angle_{i j}=\angle\left[Q_{i} \pitchfork Q_{j}\right]$ are constant on all $(n-2)$-faces of $P$.

For example, the domains $P$ in spaces of constant curvature which are bounded by (convex or concave) umbilical hypersurfaces (where all principal curvatures are constant and mutually equal, e.g. as it is for spheres) are PBhedra.

The combinatorially simplest PB-hedra are $d i$ - $B$-hedra with two $(n-1)$-faces $Q_{1}$ and $Q_{2}$ meeting across a single $(n-2)$-face $Q_{12}=Q_{1} \cap Q_{2}$. Probably, the space of $d i$ - $B$-hedra $P \subset X$ with $Q_{12}$ contained in a hypersurface $H=H^{n-1} \subset X$ is Fredholm, i.e. it locally has finite, positive or negative, "virtual dimension" $d$ : if $\mathcal{H}$ is a generic $N$-dimensional family of hypersurfaces in $X$ with large $N$, then the space $\mathcal{P}$ of di-B-hedra $P \subset X$ which are close to a given $P_{0}$ and have $Q_{12}$ contained in some $H \in \mathcal{H}$, satisfies $\operatorname{dim}(\mathcal{P})=N+d$. (Possibly, this $d$ may depend on whether a hypersurface $H$ separates $Q_{1}$ and $Q_{2}$ or not.)

The geometric type of a $P$ is, by definition, the totality of the numbers $\left\{M_{i}, \alpha_{i j}\right\}$ for $M_{i}=m n . c u r v\left(Q_{i}\right)$ and $\alpha_{i j}=\angle\left[Q_{i} \pitchfork Q_{j}\right]$ associated to the $(n-1)$ faces and $(n-2)$-faces of (the combinatorial scheme of) $P$. 
$\mathcal{P B}$-Problems. The space $\mathcal{P} \mathcal{B}(X)_{\mathcal{G} \mathcal{T}}$ of $P B$-hedra of a given geometric type $\mathcal{G} \mathcal{T}$ (which includes the combinatorial type) in a given Riemannian manifold $X=X^{n}$ is similar in many respects to the space $\mathcal{M I N}(X)$ of closed minimal hypersurfaces in $X$, albeit the spaces $\mathcal{P B}(X)_{\mathcal{G}} \mathcal{T}$ are infinite dimensional at certain $P \in \mathcal{P B}(X)_{\mathcal{G} \mathcal{T}}$ and it may be hard to decide, for example, if $\mathcal{P B}(X)_{\mathcal{G}}$ is non-empty for given $X$ and $\mathcal{G} \mathcal{T}$.

Also, the compactness properties of (subspaces in) $\mathcal{P B}(X)_{\mathcal{G}} \mathcal{T}$ are less apparent than these in $\mathcal{M I N}(X)$, since sequences of PB-hedra $P_{i} \in \mathcal{P B}(X)_{\mathcal{G}} \mathcal{T}$ may Hausdorff converge to subsets $Z \subset X$ with $\operatorname{dim}(Z) \leq n-1$, where the picture is not fully clear even for decreasing sequences $P_{0} \supset P_{1} \supset P_{i} \supset \ldots$ where the intersection $Z=\bigcap_{i} P_{i}$ may be(?) rather complicated, say for sequences of acute Plateau-hedra of the combinatorial type of the $n$-cube. For example,

What are smooth $k$-dimensional submanifolds $Z \subset X$ with boundaries which can be represented as such intersections $\bigcap_{i} P_{i}$ ?

Another new feature of Plateau-hedra (and of more general PB-hedra) is their dependence on the underlying combinatorial scheme $\mathcal{G} \mathcal{T}$ : the space $\mathcal{P B}(X)$

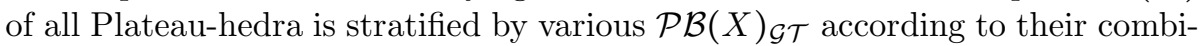
natorial types, (similarly to the space of convex Euclidean polyhedra), but the topology/geometry of this stratification is far from transparent.

The concepts of dihedral extremality and rigidity we met earlier obviously extend (as in the problem $(*)$ stated at the end of section 2.2) to PB-hedra $P$ in general Riemannian manifolds $X$ where they seem particularly interesting in manifolds with constant curvature and where they (partly) generalize rigidity phenomena for hyperbolic warped products [19].

\subsection{Multi-bubble Description and Construction of PB- hedra.}

Let $\mathcal{P}_{\mathcal{C T}}$ be the space of polyhedral domains $P$ in $X$ with $m$ faces meeting according a given combinatorial pattern (scheme/type) $\mathcal{C} \mathcal{T}$.

Denote by $v_{i}=v_{i}(P), P \in \mathcal{P}_{\mathcal{C} \mathcal{T}}$, the $(n-1)$-volume form (measure) on the face $Q_{i}$ of $P, i=1, \ldots m$, regarded as the measure on $X$.

Let $-\infty<\varepsilon_{i}<\infty$ and $-1<c_{i j}<1$ be given constants, let

$$
\mu_{i}=\varepsilon_{i} \cdot v l_{n}+\sum_{j} c_{i j} v_{j}
$$

where $\operatorname{vol}_{n}$ is the Riemannian volume (measure) in $P$ and where the sum is taken over the faces $Q_{j}$ adjacent to $Q_{i}$.

It is clear that:

if, for a given $i$, the face $Q_{i}$ is a local $\mu_{i}$-bubble, i.e. if $Y_{i}$ locally minimizes the area $_{-\mu_{i}}\left(Y_{i}\right)$ then $Q_{i}$ has constant mean curvature $=\varepsilon_{i}$ and the dihedral angles between $Q_{i}$ and $Q_{j}$ equal $\arccos \left(c_{i j}\right)$ for all $Q_{j}$ adjacent to $Q_{i}$,

$$
\operatorname{mn.curv}\left(Q_{i}\right)=\varepsilon_{i} \text { and } \cos \left(\angle\left[Q_{i} \pitchfork Q_{j}\right]\right)=c_{i j} \text {. }
$$

Minimal PB-hedra. Let $P^{\circ}$ be a Riemannian manifold with corners, e.g. a polyhedral domain in a manifold $X=X^{n}$, such that the mean curvatures of the 
$(n-1)$-faces $Q_{i}^{\circ} \subset P^{\circ}$ and the dihedral angles between them satisfy

$$
\text { mn.curv }\left(Q_{i}^{\circ}\right) \geq \varepsilon_{i} \text { and } \angle\left(Q_{i}^{\circ} Q_{j}^{\circ}\right) \leq \alpha_{i j} \text { for given } \varepsilon_{i} \geq 0 \text { and } \alpha_{i j} \leq \pi / 2
$$

and let

$$
\mu_{i}=\varepsilon_{i} \cdot \operatorname{vol}_{n}+\sum_{j}\left(\cos \alpha_{i j}\right) v_{j} .
$$

Now, take some $i_{1}$ and minimize the the $\mu_{i_{1}}$-area of of the $i_{1}$-th $(n-1)$-face by varying $Q_{i_{1}}^{\circ} \leadsto Q_{i_{1}}$ within $P^{\circ}$ and with the boundary $\partial Q_{i_{1}}$ contained in the union of the $(n-1)$-faces adjacent to $Q_{i_{1}}^{\circ}$ while keeping all $(n-1)$-faces $Q_{i \neq i_{1}}^{\circ}$ unchanged.

In other words, consider all closed subsets $P \subset P^{\circ}$ with m.c. convex boundaries $\partial P$ that contain all faces of $P^{\circ}$ which do not intersect $Q_{i}^{\circ}$, and then minimize the $\mu_{i_{1}}$-area of the new part of $\partial P$, that is $Q_{i_{1}}=\partial P \backslash \partial P^{\circ}$.

Thus we obtain some subset, say $P^{\left[i_{1}\right]} \subset P^{\circ}$ which we regard as m.c. polyhedron with a minimal $i$-th face $Q\left[i_{1}\right]_{i_{1}}=Q_{i}^{\text {min }}$ and with new faces $Q_{i}^{\left[i_{1}\right]}=$ $P^{\left[i_{1}\right]} \cap Q_{j}$ for $i \neq i_{1}$.

This $P^{[i]}$ can be more singular than it is allowed by our definition of "polyhedral domain", but we pretend it is such a domain and then apply the same minimization process to $P^{\left[i_{1}\right]}$ with respect to some face $Q_{i_{2}}^{\left[i_{1}\right]}$ for $i_{2} \neq i_{1}$.

By continuing this process with a given sequence $i_{1}, i_{2}, \ldots$ one arrives at a decreasing family of mean curvature convex "polyhedra"

$$
\ldots \subset P^{\left[i_{1}, i_{2}, i_{3}\right]} \subset P^{\left[i_{1}, i_{2}\right]} \subset P^{\left[i_{1}\right]} \subset P^{\circ}
$$

If this family stabilizes at some $P_{\min }(k)=P^{\left[i_{1}, i_{2}, i_{3}, \ldots i_{k}\right]}$ and this $P_{\min }(k) c$ $X$ qualifies as a polyhedral domain, it serves as a minimal PB-hedron inside $P^{\circ}$.

\section{Plateau Traps, $\phi$-Convexity, Qiasiregularity and Regularization.}

Let $P$ be a cubical polyhedral domain (or a manifold with corners) let $\pm Q \subset P$ be a pair of opposite faces and $X=P \backslash(Q \cup-Q)$ be the cornered manifold with the boundary $\partial X=\partial P \backslash(Q \cup-Q)$ that is the union of all $(n-1)$-faces except $\pm Q$.

If $P$ has strictly acute dihedral angles and strictly mean (curvature) convex faces, then the minimum of the $(n-1)$-volumes of hypersurfaces $Y \subset X$ with $\partial Y \subset \partial X$ is assumed by some $Y_{\text {min }}$ away from these faces inside $P^{\prime}$.

One may say that the relative homology class $[Y] \in H_{n-1}(X, \partial X)$ is trapped between $Q$ and $-Q$.

The trapping feature, if shared by all pairs of opposite faces in $P$, is equivalent to the [acute angles + mean convexity] property of $P$. On the other hand, traps are quite robust. In particular the definition of traps needs significantly less regularity than that of mean curvature, be it the topology of $P$, its Riemannian metric or smoothness assumption on the faces of $P$.

In what follows we review simple standard properties of traps; we limit ourselves for the most part of the exposition to closed hypersurfaces representing absolute $(n-1)$-homology classes in order not to overburden our notation. 


\subsection{Directed Homology.}

Let $X$ be an $n$-manifold, possibly with a boundary. A direction $\stackrel{\leftrightarrow}{C}_{n-1}$ in $X$ is, by definition, a (directing) homology class which is representable by a closed cooriented hypersurface $Y \subset X \backslash \partial X$ which divide $X$ into two closed subsets denoted $U_{\text {in }} \subset X$ and $U_{\text {out }} \subset X$ where

$$
\partial U_{\text {in }}=\partial U_{\text {out }}=Y=U_{\text {in }} \cap U_{\text {out }} .
$$

Remark. If $X$ is oriented, then $\stackrel{\leftrightarrow}{C}_{n-1}$ is an ordinary homology class, but, in general, it is, strictly speaking, a 1-cohomology class. We say "homology" to emphasize geometric representations of $\stackrel{\leftrightarrow}{C}_{n-1}$ by hypersurfaces even for nonorientable $X$, e.g. where $X=Y \times \mathbb{R}$ and $Y$ is non-orientable.

Relative Case. Let $\partial_{\circ} X \subset \partial X$ be an open subset in the boundary of $X$, e.g. $\partial_{\circ} X=\partial X$. Then there is an obvious generalization of the above to hypersurfaces $Y \subset X$ with $\partial Y \subset \partial_{\circ} X$.

The directing homology class is represented in the general relative case by non-closed cooriented hypersurfaces $Y$ that are still are closed as subset in $X$ and that may have boundaries contained in the $\partial_{\circ} X$-region of the boundary of $X$.

If all infinity of our $Y$ is contained in $\partial_{\circ} X$, everything can be reduced to the absolute case by taking the double of $X$ along $\partial_{\circ} X$. On the other hand, such doubling does not (quite) apply to a more general setting, e.g. where $X$ has no boundary at all and where $\partial_{\circ} X$ is a "virtual subset at infinity" represented by a descending family of open subsets in $X$, say $U_{1} \supset U_{2} \supset \ldots \supset U_{i} \supset \ldots$, such that the intersection of every compact subset $K \subset X$ with $U_{i}$ is empty for all $i \geq i(K)$.

The relevant hypersurfaces $Y \subset X$ here are those where the difference sets $Y \backslash U_{i}$ are compact for all $i$.

Given a Riemannian metric on $X$ and a Borel measure $\mu$, define as earlier

$$
\operatorname{area}_{\mu}(Y)=\operatorname{vol}_{n-1}(Y)-\mu[\ldots \stackrel{\text { in }}{\leftarrow} Y] \text { for } n=\operatorname{dim}(X) \text {, }
$$

for all cooriented $Y$ in the class $\stackrel{\leftrightarrow}{C}_{n-1}$ as we did in the previous section.

\subsection{Traps and Walls}

A domain $U \subset X$, is called a $\mu$-trap (or well) for $\stackrel{\leftrightarrow}{C}_{n-1}$ in $X$ and its boundary is called a $\mu$-wall if $\stackrel{\leftrightarrow}{C}_{n-1}$ can be represented by a smooth cooriented hypersurface in the interior $\operatorname{int}(U)$ and if every smooth cooriented hypersurface $Y \subset U$ which represents $\stackrel{\leftrightarrow}{C}_{n-1}$, i.e. $[Y]=\stackrel{\leftrightarrow}{C}_{n-1}$, (such a $Y$ separates the two $\stackrel{\leftrightarrow}{C}_{n-1}$-ends in $X$ ) can be "moved" to $Y^{\prime} \subset \operatorname{int}(U)$, such that

$$
\operatorname{area}_{-\mu}\left(Y^{\prime}\right) \leq \operatorname{area}_{-\mu}(Y)
$$

where "moved" signifies that $Y^{\prime}$ is a smooth cooriented closed hypersurface homologous to $Y$, i.e. $\left[Y^{\prime}\right]=[Y]=\stackrel{\leftrightarrow}{C}_{n-1}$ and this inequality is sharp unless $Y \subset \operatorname{int}(U)$ to start with. 
If $\mu=0$ these are called Plateau traps/walls or just traps and walls; if $\mu=\phi d x$ we speak of $\phi$-traps and $\phi$-walls for functions $\phi$ on $X$.

Remarks. (a) The topologically simplest situation, and this is the one we mostly deal with, is where $X$ is compact with two boundary components, e.g. a cylinder $X=Y \times[0,1]$, and where our hypersurfaces in $X$ separate these components.

(b) More general singular measures $\mu$, e.g. those supported on the boundary $\partial X$ are relevant for PB-hedra and the corresponding traps for the relative homology $H_{n-1}\left(X, \partial_{\circ} X\right)$ for some subset $\partial_{\circ} X \subset \partial X$.

Smooth Mean Convex Traps. If the boundary of a domain $U$ has strictly positive mean curvature then $U$ it traps all classes $\stackrel{\leftrightarrow}{C}_{n-1}$ that have representative cycles in $U$. More generally (and equally obviously), if $U$ is preconvex and the mean curvatures of the faces are strictly bounded from below by a function $\phi(x)$ then $U$ is $\phi$-traps.

Normal Traps. Let $Y \subset X$ be a closed smooth cooriented hypersurface that represent a non-zero directed homology class in $X$ and $\phi(x)$ be a $C^{1}$-smooth function such that $\phi(y)=m n . \operatorname{curv}_{y}(Y)$ for all $y \in Y$.

If the inward normal derivative $\frac{d \phi(y)}{d \nu^{i n}}$ on $Y$, (for $\nu^{i n}$ being the inward looking unit normal vector along $Y$ ) is sufficiently large, say

$$
\frac{d \phi(y)}{d \nu_{y}^{i n}}>\operatorname{curv}_{y}^{2}(Y)+\left|\operatorname{Ricci}_{X}\left(\nu_{y}^{i n}, \nu_{y}^{i n}\right)\right| \text { for all } y \in Y,
$$

where $c u r v^{2}$ denotes the sum of squares of the principal curvatures of $Y$ and where, observe, $\operatorname{Ricci}\left(\nu^{\text {in }}, \nu^{\text {in }}\right)=\operatorname{Ricci}\left(\nu^{\text {out }}, \nu^{\text {out }}\right)$, then

the homology class $\stackrel{\leftrightarrow}{C}_{n-1}=[Y]$ in $U_{0}$ is $\phi$-trapped in all neighbourhoods

$U \subset X$ of $Y$.

Indeed, let $d(x)$ be a smooth function in a neighborhood of $Y$ without critical points that is negative inside $Y$, i.e. on $[\ldots \stackrel{\text { in }}{\leftarrow} Y]$, and positive on $[Y \stackrel{\text { out }}{\leftarrow} \ldots]$. If the mean curvatures of the levels of this function cooriented as $Y$ strictly minorize $\phi(x)$ strictly inside $Y$ i.e. for $x \in[\ldots \stackrel{\leftrightarrow}{Y}] \backslash Y$ and strictly majorize $\phi(x)$ strictly outside $Y$, then, obviously the homology class of $Y$ is trapped in the domain $d^{-1}[-\varepsilon, \varepsilon] \subset X$ for all (arbitrarily small) $\varepsilon>0$.

If this applies to the signed distance function $d(x)= \pm(x) \operatorname{dist}(x, Y)$ negative inside $Y$ and positive outside, then the sufficiency of the above lower bound on $\frac{d \phi(y)}{d \nu_{y}^{i n}}$ follows from the second variation formula for $\operatorname{vol}_{n-1}(Y)$.

Locally Trapped Hypersurfaces with Boundary. Let $Y$ be a smooth compact hypersurface with a boundary $\partial Y \subset \partial X$, let $\phi_{\circ}: \partial X \rightarrow[-1+\beta, 1-\beta], \beta>0$, be a $C^{1}$-smooth function and let $\mu=\phi \cdot \operatorname{vol}_{n}+\phi_{\circ} v{ } l_{n-1}$ for $\operatorname{vol}_{n-1}$ referring here to the Riemannian volume (measure) on the boundary $\partial X$.

Let the (dihedral) angle $\angle_{y}={ }_{y}(Y \pitchfork \partial X)$ satisfy

$$
\cos \angle_{y}=\phi_{\mathrm{o}}(y) \text { for all } y \in \partial Y
$$

If the inward normal derivative $\frac{d \phi(y)}{d \nu^{i n}}$ is sufficiently large, where the lower bound depends, besides curv ${ }^{2}(Y)$ and Ricci $(X)$, on $\operatorname{curv}^{2}(\partial Y)$, on $\left(1-\left|\phi_{\circ}\right|\right)^{-1} \leq$ $\beta^{-1}$, and on the normal derivative of $\phi_{\circ}$ in $\partial Y$, then $Y$ minimizes are $a_{\mu}$ in every small neighbourhood $U_{0} \supset Y$. 


\subsection{Smoothing and Doubling.}

Let $U$ be a compact domain in a smooth Riemannian $n$-manifold $X \supset U$ that traps a directed homology class $\stackrel{\leftrightarrow}{C}_{n-1}$. Then

there exists a subdomain $U^{\prime} \subset U$ with $C^{2}$-smooth boundary $Y^{\prime}$ that also $\phi(x)$ traps $\stackrel{\leftrightarrow}{C}_{n-1}$ and such that the mean curvatures of $Y^{\prime}$ satisfy

$$
\text { mn.curv }{ }_{x}\left(Y^{\prime}\right)>0 \text { for all } x \subset Y^{\prime} \text {. }
$$

Proof. Since the class $\stackrel{\leftrightarrow}{C}_{n-1}$ is trapped in $U$ it can be realized by, a priori singular, cooriented hypersurface $Y_{\min } \subset \operatorname{int}(U)$.

This $Y_{\min }$ may be non-unique; in this case we take the union of all these minimal $Y_{\min }$, denoted $Y_{M I N}=\cup Y_{\min }$ that is a compact subset in the interior of $U$.

Clearly, the class $\stackrel{\leftrightarrow}{C}_{n-1}$ is trapped in all neighborhoods $V \subset U$ of $Y_{M I N}$, and, by continuity, the class class $\stackrel{\leftrightarrow}{C}_{n-1}$ is $\varepsilon$-trapped in $V$ for all, positive and negative $\varepsilon=\varepsilon(V)$ with sufficiently small absolute values $|\varepsilon|>0$. Therefore, $Y_{M I N}$ is pinched between two $\varepsilon$-bubbles, $\varepsilon>0$, i.e. it is contained in a domain $V_{\varepsilon}^{\prime} \subset V$ bounded by these bubbles.

We conclude the proof by smoothing these bubbles keeping their mean curvatures positive (see [22] and thus, approximate $V^{\prime}$ by the required smooth domain $U_{\varepsilon}^{\prime}$. QED

Scalar Curvature Corollary. Let a domain $U$ that traps a homology class $\stackrel{\leftrightarrow}{C}_{n-1}$ admit a continuous map to the $(n-1)$-torus, say $f: U \rightarrow \mathbb{T}^{n-1}$, such that $\stackrel{\leftrightarrow}{C}_{n-1}$ goes to a non-zero multiple of the fundamental class of the torus. If $U$ is spin, then

the scalar curvature of $X$ is strictly negative at some point $x \in U$.

Proof. As one knows (see 23 that if $\operatorname{scal}\left(U_{\varepsilon}^{\prime}\right) \geq 0$, then the double, say $U_{\varepsilon}^{\prime+\prime}$ of $U_{\varepsilon}^{\prime}$ is a closed manifold that admits a smooth metric with positive scalar curvature. Then the proof follows from the validity of the Geroch conjecture in the spin case, 23 .

Remarks. (a) The above applies whenever the topology of (the closed manifold) $U_{\varepsilon}^{\prime+\prime}$ does not allow metrics on it with scal $>0$ on it that is known in a variety of cases. In particular, the Schoen-Yau theorem allows one to suppress the spin condition for $n \leq 7$.

It was conjectured by Brian White (a private communication about 20-25 years ago) that

$$
\text { singularities of minimal hypersurfaces are unstable. }
$$

This would allow an extension of the Schoen-Yau method to all $n$ (with some problems remaining in proving rigidity results).

White's conjecture was confirmed in [4] for $n=8$, where, observe, singularities are isolated.

The case $n \geq 9$ remains open, but we shall see in section 5.3 how to extend Schoen-Yau results to non-spin manifolds of dimension $n=9$ where the singularities $\Sigma$ of minimal hypersurfaces are most 1-dimensional. 
Furthermore, Lohkamp's method of "going around singularities" applies to all $n$, but I have not studied it in depth and can not apply it to the problems at hand.

(b) Generically $Y_{\min }$ is unique and then the (smooth mean curvature convex) domain $U_{\varepsilon}^{\prime} \supset Y_{\min }$ can be chosen arbitrarily close to $Y_{\min }$.

The geometry of such $U_{\varepsilon}^{\prime}$ is similar to the warped product metric on $Y \times \mathbb{R}$ from [25] Apparently, the former converges in a suitable sense to the latter for $\varepsilon \rightarrow 0$ as the neighborhood $U_{\varepsilon}^{\prime}$, that converges to $Y_{\text {min }}$, becomes "infinitesimally narrow".

In fact the arguments using the warped product from [25] can be trivially adjusted to $U_{\varepsilon}^{\prime}$ and/or to its double $U_{\varepsilon}^{\prime+\prime}$.

The advantage of this over the warped product is that it make sense for singular $Y_{\min }$ but we have only managed a limited use of it (such as handling dimensions $n=8,9$ for non-spin manifold).

Also $U_{\varepsilon}^{\prime}$ it is harder (but, probably, possible) to use it with bubbles $Y$ in spaces with negative scalar curvature as we did in [19] with a help of warped products.

Smoothing at the Edges. Let $P$ be a singular polyhedral domain in a Riemannian manifold $X$, i.e. where the faces may have singularities of the kind minimal hypersurfaces have.

For instance, Let $P$ be bounded by a pair of hypersurfaces, say $W_{0}$ and $W_{1}$ where $W_{0}$ is a minimal hypersurface and $W_{1}$ is a $\phi$-bubble with free boundary $\partial W_{1} \subset W_{0}$ for a continuous function $\phi$ on $X$.

The dihedral angle between $W_{0}$ and $W_{1}$ equals $\pi / 2$ at the points $x$ at the edge $\partial W_{1}=W_{0} \cap W_{1}$ where both $W_{0}$ and $W_{1}$ are regular.

But when it comes to singular points it is not even clear if the concept of dihedral angle is symmetric, i.e. if $\angle\left(W_{0}, W_{1}\right)=\angle\left(W_{1}, W_{0}\right)$.

Both $W_{0}$ and $W_{1}$ can be approximated by smooth hypersurfaces with a minor decrease of their mean curvatures 22 (in fact, with an increase if $\operatorname{Ricci}(X) \geq 0$ ). Such a smoothing starts with a small equidistant inward deformation of $W_{0}$ and $W_{1}$. If $W_{0}$ and $W_{1}$ are smooth at the edge points, then the dihedral angle also changes little but the dihedral angle may, a priori, uncontrollable increase near the singularities in the edge.

Question. Is it possible to smooth the faces, with at most $\varepsilon$-decrease of their mean curvatures and with at most $\varepsilon$-increase of the dihedral angle(s) for an arbitrarily small $\varepsilon>0$ ?

\section{$4.4 \Delta$-Stable Mean Convexity.}

We want to define a concept of mean curvature bounded from below, in particular, of (strictly and non-strictly) positive mean curvature for non-smooth domains $U \subset X$ and want this positivity to be stable under small continuous perturbations of $U$ as well as under $C^{0}$-perturbations of the Riemannian metric in $X$. Eventually, we want a concept adaptable to singular spaces. There are several candidates for such a stable mean convexity. Below is a instance of such a definition that depends on Almgren's concept of

$\varepsilon$-Minimization. Let $H \subset X$ be a smooth cooriented hypersurface, let $H_{0} \subset H$ a compact domain in $H$ and $H_{1}=H \backslash H_{0}$. 
$A$ (smooth) $\varepsilon$-minimization of $H$ supported in $H_{0}$, denoted $H \leadsto H^{\prime}$, is a replacement of $H$ by another smooth cooriented hypersurface $H^{\prime}$ such that $H^{\prime}=$ $H_{0}^{\prime} \cup H_{1}^{\prime}$, where $H_{1}^{\prime}=H_{1}$, where the union $H_{0} \cup H_{0}^{\prime}$ makes a closed hypersurface that bounds in $X$ (and so $H^{\prime}$ is homologous to $H$ ) and such that

$$
\operatorname{vol}_{n-1}\left(H_{0}^{\prime}\right) \leq e^{-\varepsilon} \operatorname{vol}_{n-1}\left(H_{0}\right)
$$

where we assume, to avoid immaterial complications, that the boundary of $H_{0}$ in $H$ has Hausdorff dimension at most $n-2$.

Clearly,

composition of $\varepsilon$-minimizations

$$
H \leadsto H^{\prime} \leadsto H^{\prime \prime}
$$

is an $\varepsilon$-minimization

$$
H \leadsto H^{\prime \prime}
$$

the support of which equals the union of the supports of its factors.

Also observe that this minimization is stable under small perturbations of the Riemannian metric $g$ on $X$ :

if

$$
\lambda^{-2} g<g_{1}<\lambda^{2} g \text { for } \lambda^{n-2} \leq e^{\varepsilon}
$$

then $H \leadsto H^{\prime}$ is an $\varepsilon_{1}$-minimization in $\left(X, g_{1}\right)$ for $\varepsilon_{1}=\varepsilon-(n-2) \log \lambda$.

$\Delta$-Stable Mean Convexity. Let $U$ be a closed domain in $X$ with compact boundary $Y=\partial U$, let $V_{1}, V_{2} \subset U$ be closed subsets in $U$ that contain $Y$ and such that $V_{1}$ is contained in the interior of $V_{2}$; let $\Delta=V_{2} \backslash V_{1}$.

Say that $Y$ is (multiplicatively) $(\varepsilon, \Delta)$-stably mean convex if there exists a (small) neighbourhood $W \subset V_{2}$ of $Y \subset V_{2}$ such that every smooth cooriented hypersurface $H \subset V_{2}$ that intersect $V_{1}$ admits an $\varepsilon$-minimization $H \leadsto H^{\prime}$, such that

$\bullet_{1} H^{\prime}$ does not intersect $W$;

$\bullet_{2}$ the support $H_{0} \subset H$ of the $\varepsilon$-minimization $H \leadsto H^{\prime}$ is contained in $V_{2}$;

- 3 the hypersurfaces $H$ and $H^{\prime}$ coincide outside $V_{2}$, i.e. the "new part"

$H_{0}^{\prime} \subset H^{\prime}$ of $H^{\prime}$ is contained in $V_{2}$.

Remark. What is most essential here is that the volume of $H_{0}^{\prime}$ is smaller than that of $H_{0}$ by a definite amount, (roughly by $\varepsilon \cdot v l_{n-1}\left(H_{0}\right)$ ) that is independent of $H$ : this makes this mean convexity stable under small perturbation of the metric in $X$.

On the other hand, keeping $H^{\prime}$ away from $Y$ is a minor issue since boundaries of domains, as we define them, are large. (Nothing like $U=X \backslash Z$ with $\operatorname{codim}(Z) \geq 2$ is allowed.) Yet, this is needed to make this convexity stable under small perturbation of $Y$.

(*) Mean Convex $\Rightarrow \Delta$-Stable Mean Convex. Let $U$ be a domain in a complete Riemannian manifold $X$, denote by $U_{-\delta} \subset U$ the subset of those $u \in U$ where $\operatorname{dist}(u, Y) \geq \delta$ and let $Y_{-\delta}=\partial U_{-\delta}$ be the corresponding equidistant hypersurfaces.

Let $Y$ has strictly positive mean curvature and observe that $Y_{-\delta}=\partial U_{-\delta}$ also have positive mean curvatures for small $\delta<\delta_{0}=\delta_{0}(Y)>0$.

There exists an $\varepsilon=\varepsilon(U, \delta)>0$ for all positive $\delta<\delta_{0}$ such that $U$ is $(\varepsilon, \Delta)$ stably mean convex for for $\Delta=U_{-\delta_{1}} \backslash U_{-\delta_{2}}$ and all $0<\delta_{2}<\delta_{1} \leq \delta$. 
Proof. One may assume (this is easy, compare 3, 15]) that $H \cap V_{2}$ is $\varepsilon$ minimal that is no $\varepsilon$-minimization (for a suitably small $\varepsilon>0$ ) that satisfies the above $\bullet_{2}$ and $\bullet_{3}$ exists. This property provides a lower bound on the $(n-1)$ volumes of $H$ intersected with $\rho$-balls $B_{h}(\rho) \subset \Delta$, see [3]. It follows, that there exists $\delta^{\prime}$ in the interval $\delta_{2}<\delta^{\prime}<\delta_{1}$ such that the region $H_{0}^{\prime} \subset Y_{-\delta^{\prime}}$ bounded in $Y_{-\delta^{\prime}}$ by $H \cap Y_{-\delta^{\prime}}$ satisfies:

$$
\operatorname{vol}_{n-2}\left(H_{0}^{\prime}\right) \leq e^{-\varepsilon} v l_{n-2}\left(H_{0}\right) \text { for } H_{0}=H \cap\left(U \backslash U_{-\delta^{\prime}}\right) .
$$

QED.

(**) $\Delta$-Stable Mean Convexity $\Rightarrow$ Mean Convexity. Let a closed domain $U \subset X$ be $(\varepsilon, \Delta)$-stably mean convex for some $\Delta=V_{2} \backslash V_{1} \subset U$ and $\varepsilon>0$.

Then there exists a smooth mean curvature convex subdomain $U_{1}$ in $U$ such that $V_{1} \subset U_{1} \subset V_{2}$; thus, the (compact smooth mean curvature convex) boundary $Y_{1}=\partial U_{1}$ is contained in $\Delta$.

Proof. Let $\phi(x)$ be a non-negative continuous function that is very small on $V_{1}$ and that fast grows in $\Delta$ as $x$ approaches the complement $U \backslash V_{2}$. Then, the $\Delta$-stable mean convexity of $U$ implies the existence of a $\phi$-bubble trapped inside $\Delta$ and the required $Y_{1}$ is obtained with a smooth approximation of this bubble, see 22].

Remark/Question. This arguments relies on the basic regularity theorems of the geometric measure theory. Is there an elementary proof of the implication $(* *)$ ?

On Relative $\Delta$-Stable Mean Convexity. The definition of $\Delta$-stable mean convexity obviously generalizes to the relative case where (possibly non-smooth) hypersurfces $Y$ have boundaries that must be contained in a given closed subset $W \subset X$. The so defined notion of "mean convexity" is stable under small perturbations of $Y$ and of the metric $g$ on $X$, but... not under $C^{0}$-perturbation of $W$.

For instance, if $W$ is a smooth closed hypersurface in $X$ that bounds a domain $U \subset X$ and $W_{\varepsilon}$ is obtained by a smooth diffeotopy that keeps $W_{\varepsilon}$ all along in a small $\varepsilon$-neighbourhood of $W$ in $X$, then the local geometry of $X$ near $W_{\varepsilon}$ is not necessarily close in any sense to the local geometry of $X$ near $W$. Because of this, even if $Y \subset U$ is locally isolated minimal submanifold with (free) boundary $\partial Y \subset W=\partial U$, one can not guarantee that the minimal $Y_{\varepsilon} \subset U_{\varepsilon}$ with $\partial Y_{\varepsilon} \subset W_{\varepsilon}$ will be, in general, geometrically close to $Y$.

Of course, the bulk of $Y_{\varepsilon}$ away from $W_{\varepsilon}$ and also for most of the boundary of $Y_{\varepsilon}$ will be close to $Y$ but if the local relative filling profile of $U_{\varepsilon}$ near $W_{\varepsilon}$ becomes badly non-Euclidean, then $Y_{\varepsilon}$ may have develop "long narrow fingers" in vicinity of $W_{\varepsilon}$ protruding somewhere at the boundary of $Y_{\varepsilon}$ and spearing in $U_{\varepsilon}$ along its boundary $W_{\varepsilon}$.

However, we shall see presently that if the hypersurface $W_{\varepsilon}$ serves as a bubble for a $C^{0}$-small $\varepsilon$-perturbation $g_{\varepsilon}$ of the original Riemannian metric $g$ in $X$, then

the domain $U_{\varepsilon} \subset X$ bounded by $W_{\varepsilon}$ satisfies almost Euclidean filling

inequalities for relative $(k-1)$-cycles $C \subset U_{\varepsilon}$ for all $k=2,3, \ldots, \operatorname{dim}(X)-1$.

This rules out "fingers" and guarantees the existence of a relative $g$-bubble $Y_{\varepsilon} \subset U_{\varepsilon}$ with $\partial Y_{\varepsilon} \subset W_{\varepsilon}=\partial U_{\varepsilon}$ that approximate a given submanifold $Y \subset U$ with $\partial Y \subset \partial U$. 
Furthermore, if $U_{\varepsilon}^{\prime} \subset U_{\varepsilon}$ equals a part of $U_{\varepsilon}^{\prime}$ bounded by $Y_{\varepsilon}$ and a "half" of the boundary of $U_{\varepsilon}$, then one can, in some cases, construct bubbles $Y_{\varepsilon}^{\prime} \subset U_{\varepsilon}^{\prime}$ with boundaries in $\partial U_{\varepsilon}^{\prime}$, where, observe the boundary $\partial U_{\varepsilon}^{\prime}$ has a corner along the set where $Y_{\varepsilon}$ meets $W_{\varepsilon}=\partial U_{\varepsilon}$ and this corner may necessitate a presence of a singularity in $Y_{\varepsilon}^{\prime}$ at this corner.

The construction of such bubbles $Y_{\varepsilon}, Y_{\varepsilon}^{\prime}, Y_{\varepsilon}^{\prime \prime}, \ldots$ will be used for proving that $C^{0}$-limits $g$ of metrics $g_{\varepsilon}$ with $\operatorname{scal}\left(g_{\varepsilon}\right) \geq 0$ also have $\operatorname{scal}(g) \geq 0$.

We shall do it in section 4 where we shall explain how to compensate for possible singularities of the solutions of the Plateau problem with free boundary at the corners.

\section{5 $C^{0}$-Stability of the Mean Curvature and Reifenberg Flatness.}

The above implications $(*)$ and $(* *)$ show that strictly mean convex hypersurfaces are $C^{0}$-stable but, in fact, the following more general property holds [21].

Let $Y$ be a smooth closed cooriented hypersurface in a smooth Riemannian manifold $X=\left(X, g_{0}\right)$. This $Y$ can be seen as a $\phi$-bubble for a smooth function $\phi(x)$ that extends the function $y \mapsto m n . \operatorname{curv}_{y}(Y)$; moreover, if $\phi$ has a sufficiently large normal derivative on $Y$, then, as we know, the homology class $[Y]$ is $\phi$-trapped near $Y$.

Therefore, if a metric $g_{\varepsilon}$ is sufficiently $C^{0}$ close to $g_{0}$ then a small neighborhood of $Y$ in $X$ contains a $\phi$-bubble, say $Y_{\varepsilon} \subset X$ for the metric $g_{\varepsilon}$.

The $g_{0}$-volume of this bubble is close to that of $Y$, since, clearly

$$
\operatorname{vol}_{n-1}\left(Y_{\varepsilon}\right) \rightarrow \operatorname{vol}_{n-1}(Y) \text { for } \varepsilon \rightarrow 0 \text {; }
$$

moreover, the volumes of $Y_{\varepsilon}$ within all $R$-balls in $X=\left(X, g_{0}\right)$ are also close to the volume of the balls in $Y$,

$$
\left|\operatorname{vol}_{n-1}\left(B_{y}(R) \cap Y_{\varepsilon}\right)-\operatorname{vol}_{n-1}\left(B_{y}(R) \cap Y\right)\right| \leq \delta=\delta(\varepsilon) \rightarrow 0 \text { for } \varepsilon \rightarrow 0
$$

for all $y \in Y_{\varepsilon}$ and all $R>0$.

Since the metrics $g_{\varepsilon}$ are close to a fixed continuous (smooth in our case) metric $g_{0}$, they satisfy almost Euclidean filling inequalities by Almgren's theorem [4. Consequently, small balls in the bubbles $Y_{\varepsilon}$ in $\left(X, g_{\varepsilon}\right)$ have an almost Euclidean lower bound on the volume growth.

On the other hands, small balls in $Y$ have roughly Euclidean volumes and since the metrics $g_{0}$ and $g_{\varepsilon}$ are mutually $\varepsilon$-close, the volumes of $B_{y}(\rho) \cap Y_{\varepsilon}$ are essentially the same for these metrics and the corresponding metric balls $B_{y}(\rho)$.

Then it follows by all of the above that the $g_{\varepsilon}$-volumes of $Y_{\varepsilon}$ within small balls in $X=\left(X, g_{\varepsilon}\right)$ are multiplicatively bounded by the volumes of the Euclidean balls

$\operatorname{vol}_{n-1}\left(B_{y}(\rho) \cap Y_{\varepsilon}\right) \leq\left(1+\delta^{\prime}\right) \operatorname{vol}_{n-1}\left(B_{\text {Eucl }}^{n-1}(\rho)\right)$, where $\delta^{\prime}=\delta^{\prime}(\varepsilon, \rho) \rightarrow 0$ for $\varepsilon+\rho \rightarrow 0$.

By Allard gap/regularity theorem, [1] this bounds implies regularity of $Y_{\varepsilon}$ for small $\varepsilon>0$.

Since this equally applies to the metrics $g_{t}=(1-t) g_{0}+t g_{\varepsilon}, 0 \leq t \leq 1$, one can construct a diffeotopy of smooth(!) bubbles $Y_{t} \subset\left(X, g_{t}\right)$ between $Y$ and $Y_{\varepsilon}$. 
Warning. The bubble $Y_{t}$ may be non-unique due to possible bifurcations at certain $t$ (as critical points of one parameter families of smooth functions do) and one can not guarantee smoothness of the family $Y_{t}$ in $t$.

Remark. Since $Y_{\varepsilon}$ comes by a deformation process of smooth bubbles, the standard elliptic estimates suffice for the existence of $Y_{\varepsilon}$ and the use of the geometric measure theory can be avoided at this point.

Reifenberg's Flatness. Besides being $C^{1}$-smooth, the family $Y_{\varepsilon \rightarrow 0}$ is Reifenberg flat:

the Hausdorff tangent cones of $Y_{\varepsilon \rightarrow 0}$ are isometric to $\mathbb{R}^{n-1}$ where the (abstract) Hausdorff distance of $\lambda \cdot Y_{\varepsilon}=\left(Y_{\varepsilon}, \lambda \cdot g_{0}\right)$ to $\mathbb{R}^{n-1}$ is uniformly small at all points $y$ in $Y_{\varepsilon}$ and all $\varepsilon \leq \varepsilon_{0}>0$,

$$
\operatorname{dist}_{H a u}\left(Y_{\varepsilon} \cap B_{y}(\rho), B_{E u c l}^{n-1}(\rho)\right) \leq \delta^{\prime \prime} \rho \text {, where } \delta^{\prime \prime}=\delta^{\prime \prime}\left(\varepsilon_{0}, \rho\right) \rightarrow 0 \text { for } \varepsilon_{0}+\rho \rightarrow 0 \text {. }
$$

Proof. The Hausdorff limits of $\lambda \cdot\left(Y_{\varepsilon} \cap B_{y}(\rho)\right)$ for $\lambda \rightarrow \infty$ and $\varepsilon \rightarrow 0$ are minimal hypersurfaces in $\mathbb{R}^{n}=\lim _{\text {Hau }}\left(X, \lambda g_{\varepsilon}\right)$ for $\lambda \rightarrow \infty$.

By the above, these hypersurfaces satisfy the Euclidean bound on their volume growth; hence they are flat. QED.

Remarks. (a) The Reifenberg flatness of submanifolds $Y_{\varepsilon}$ does not make them $C^{1}$-close to $Y_{0}$ for small $\varepsilon$. In fact, the $g_{0}$-normal projections $Y_{\varepsilon} \rightarrow Y$ may be many-to-one for all $\varepsilon>0$.

(b) Not all " $g_{\varepsilon}$-bubbles" close to $Y_{0}$ have their mean curvatures close to those of $Y_{0}$. For instance, there (obviously) exists an arbitrarily small $C^{0}$ perturbation $g_{\varepsilon}$ of $g_{0}$ such that $Y_{0}$ becomes a totally geodesic submanifold in $\left(X, g_{\varepsilon}\right)$, where moreover, a small neighborhood $U$ of $Y$ metrically splits $\left(U, g_{\varepsilon}\right)=$ $\left(Y,\left.g_{\varepsilon}\right|_{Y}\right) \times\left[-\delta_{\varepsilon}, \delta_{\varepsilon}\right]$. (Of course, the above $\phi$-bubbles $Y_{\varepsilon}$, albeit being close to $Y_{0}$ will not, in general, lie in such neighbourhood.)

(c) The construction of the "bubble perturbation" $Y_{\varepsilon}$ of $Y_{0}$ partly generalizes to smooth $k$-dimensional closed submanifolds $Y_{0} \subset X$ for all $k<n-1$.

For instance if $Y_{0}$ is minimal and moreover if it is an isolated local minimum for the function $Y \mapsto \operatorname{vol}_{k}(Y)$, then, clearly, a small neighbourhood of $Y$ in $X$ contains a $g_{\varepsilon}$-minimal subvariety that is non-singular and is diffeotopic to $Y_{0}$ as well as Reifenberg flat.

In general, if $Y_{0}$ is not locally minimizing, one minimizes the function

$$
Y \mapsto \operatorname{vol}_{k}(Y)-C \int_{Y} \operatorname{dist}_{X}\left(y, Y_{0}\right),
$$

where $\operatorname{vol}_{k}$ is taken with $g_{\varepsilon}$ and dist $X$ with $g_{0}$.

It is not hard to see that, if $g_{\varepsilon}$ is $C^{0}$-close to $g$, then this function has a local minimum realized by a subvariety $Y_{\min } \subset X$ that is contained in a small neighbourhood of $Y_{0}$ where it is homologous to $Y_{0}$.

Moreover, Almgren's regularity result [4] seems to imply (as it does happen for $k=n-1)$ that such a minimal $Y_{\min }$ is necessarily $C^{1}$-smooth; hence, diffeotopic to $Y_{0}$. (I am not certain about the mean curvature of this $Y_{\min }$.)

(d) Reifenberg flatness of (families of subsets) $Y_{\varepsilon}$ in smooth Riemannian manifolds $X$ implies that these are topological (actually Hölder) submanifolds by Reifenberg's topological disk theorem [?] that was extended to abstract metric space $Y$ in 9 . 
An easy result in this respect that implies the homotopy version of the disk theorem for Reifenberg (sufficiently) flat $Y_{\varepsilon} \subset X$ is the existence of smooth approximation $d(x)$ to the distance function $x \mapsto \operatorname{dist}(x, Y)$ such that the function $d(x)$ vanishes on $Y$ and has no critical points $x \in X \backslash Y$ close to $Y$ [21].

On Piecewise Smooth $C^{0}$-Mean Stability. What we needs for the proof of the $C^{0}$-stability of the inequality $\operatorname{scal}(g)<0$ is a generalization of the above to piecewise smooth $Y \subset X$ that are boundaries of mean convex polyhedral domains $U$ in $X$, where approximating $Y_{\varepsilon}$ must be also piecewise smooth and have the mean curvatures of their smooth pieces, that are $(n-1)$-faces for $Y=\partial P$, being close the mean curvatures of the corresponding pieces of $Y$ while keeping the (dihedral) angles between these pieces of $Y_{\varepsilon}$ close to the corresponding angles in $Y$.

We construct such $Y_{\varepsilon}$ by consecutively adding (hopefully) smooth faces one by one, solving at each step Plateau's free boundary problem where its solvability follows from Reifenberg kind of flatness that implies rough filling bounds needed for "cutting off" undesirable "long narrow fingers" , as we shall see in the next section.

But the solutions to such Plateau problem, say for $Y \subset U$ with boundary $\partial Y \subset \partial U$, may have singularities at the corners in $\partial U$ even if all tangent cones to $Y$ at these points are smooth, where we prove this smoothness in our case by the means of sharp Euclidean filling bounds

These singularities may be due to the failure of Allard's type regularity, which seems unlikely, or, which is more probable, they may come from the linearized Plateau.

We shall manage to "go around singularities" in our special case by a rather artificial argument in section 4.9 while the general problem of $C^{0}$-stability of mean curvature \& dihedral angles for piece-wise smooth hypersurfaces

remains open.

\subsection{Reifenberg Flatness and Filling Profile.}

$(\delta, \lambda)$-Flatness. A hypersurface $W$ in Riemannian manifold $X$ is called $(\delta, \lambda)$ flat at a point $w \in W$ on the scale $\rho$, for given positive numbers $\delta>0, \lambda \geq 1$ and $\rho>0$, if there exists a "flattening" $\lambda$-bi-Lipschitz homeomorphism $L$ from the ball $B=B_{w}(\rho) \subset X$ to the Euclidean space $\mathbb{R}^{n}, n=\operatorname{dim}(X)$, such that

- $L(w)=0 \in \mathbb{R}^{n}$;

- the image $L(W \cap B) \subset \mathbb{R}^{n}$ is contained in the $\delta \rho$-neighbourhood of the hyperplane $\mathbb{R}^{n-1} \subset \mathbb{R}^{n}$.

- the ball $B_{0}^{n-1}\left(\lambda^{-1} \rho\right) \subset \mathbb{R}^{n-1}$ is contained in the $\delta \rho$-neighbourhood of the image $L(W \cap B) \subset \mathbb{R}^{n}$.

Filling Volume. Let $U \subset X$ be a domain with boundary $W=\partial U$ and let $C \subset U$ be a relative $(k-1)$-cycle with $\partial C \subset W$

Denote by Filvol $\left._{k}(C)\right|_{U}$ the infimum of the $k$-volumes of the chains $D \subset U$ bounded by $C$, i.e. such that $\partial D \backslash Z=C \backslash Z$ and write Filvol $\left._{k}(C)\right|_{U \cap B}$ for this infimum taken over the chains $D$ that are contained in the intersection of $U$ with a given ball $B=B_{u}(r) \subset X$.

Remark. A specific local geometry of these chains and cycles is rather irrelevant for our purpose. One may think at this stage of these being realized by 
piecewise smooth subvarieties in $X$.

Let us show that if $\delta>0$ is sufficiently small depending on $\lambda \geq 1$ and if the boundary $W$ of $U$ is $(\delta, \lambda)$-flat on the scales $\leq \delta_{0}$ for a given $\rho_{0}$, then $U$ satisfies rough Euclidean filling inequality

$$
\text { Filvol }\left._{k}(C)\right|_{U} \leq \text { const }_{k, \lambda} \text { vol }_{k-1}(C)^{\frac{k}{k-1}},
$$

for all $k$-cycles $C$ of diameters $\leq 0.1 \rho_{0}$, all $k=2,3, \ldots n-1$. A precise formulation of this is as follows.

Rough Filling Inequality. There exist a continuous function $\delta_{n}(\lambda)>0$ and a constant const $t_{k} \leq(10 k)^{10 k^{2}}$ with the following properties.

Let $\rho_{0}>0$ be given and let $U \subset X$ be a domain with boundary $W$ and let $C \subset U$ be a relative $k$-cycle that is contained in the intersection of $U$ with the Riemannian ball $B_{u_{0}}\left(\rho_{0} / 10\right) \subset X, u_{0} \in U$.

If $W$ is $(\delta, \lambda)$-flat for some $\delta \leq \delta_{n} \cdot(10 \lambda)^{-(10 n)^{10 n}}$ on all scales $\rho \leq \rho_{0}$ at all points $w \in W$ within distance $10 \lambda \rho_{0}$ from $u_{0}$, where $\delta_{n}>0$ is a constant, that, in fact, may be assumed $\geq(10 n)^{-10 n}$. Then

$$
\text { Filvol }\left._{k}(C)\right|_{U \cap B} \leq \text { const }_{k} \cdot \lambda^{2 k} \operatorname{vol}_{k-1}(C)^{\frac{k}{k-1}}
$$

for the ball $B=B_{u_{0}}\left(10 \lambda \rho_{0}\right) \subset X$.

Moreover, if dist $(c, W) \leq d$ for a given $d \geq 0$ and all $c \in C$, then

$$
\text { Filvol }\left._{k}(C)\right|_{U \cap B} \leq \text { const }_{k}^{\prime} \cdot \lambda^{2 k} \cdot d \cdot \operatorname{vol}_{k-1}(C)
$$

for another universal constant const ${ }_{k}^{\prime}$.

Remarks. (a) Filling inequalities for relative cycles $C$ in $U$ with $\partial C \subset \partial U$ are equivalent to such inequalities for absolute cycles in the double of $U$, where these cycles are symmetric under the obvious involution of the double.

(b) If $W=\partial U$ is everywhere $(\delta, \lambda)$-flat then $W$ admits a collar in $U$ that is a neighbourhood homeomorphic to $W \times[0,1]$. This is seen with the smoothed distance function $u \mapsto \operatorname{dist}(u, W)$ that has no critical points away from $W$.

In general, there is no Lipschitz collar. On the other hand, Reifenberg's disc theorem says in the present context that $W$ is a Hölder $(n-1)$-manifold. (Possibly, the gradient flow of the smoothed distance function $u \mapsto \operatorname{dist}(u, Z=$ $\partial U)$ may lead to a simple poof of this.)

Proof of (1) and (2). We combine the filling argument by induction on $k$ from [15, 49] with Reifenberg's style multi-scale iteration process where the latter is amounts to proving the inequalities (1) and (2) on the scale $\rho$, i.e. for cycles contained in $\rho$-balls, provided that such inequalities with slightly different constants are valid on the scale $\delta \rho$ for some moderately small positive $\delta<1$.

Induction starts with $k=2$. One think of relative 1-cycles as $\operatorname{arcs} C \subset U$ with the ends in $W=\partial U$ and multi-scaling is especially simple.

To see this let $C \subset U \cap B$ where $B$ is a ball of radius $\rho \leq \rho_{0} / 10$ and let us "chop away" the part of $C$ that is $d$-far from $W$ for $d=2 \delta_{n} \lambda \cdot \rho$.

This done with the "flattening" homeomorphism $L: B \rightarrow \mathbb{R}^{n}$, where may assume that it sends $B \cap U$ to the half space $\mathbb{R}_{+}^{n}$.

Let $\mathbb{R}_{-\varepsilon}^{n-1} \subset \mathbb{R}_{+}^{n}$ be the hyperplane parallel to $\mathbb{R}^{n-1}=\partial \mathbb{R}_{+}^{n}$ obtained by moving $\mathbb{R}^{n-1}$ inside $\mathbb{R}_{+}^{n}$ by $\varepsilon$. We cut $C$ by the $L$-pullback $H=L^{-1}\left(\mathbb{R}_{-\varepsilon}^{n-1}\right)$ for $\varepsilon=\delta_{n} \rho$ 
and let $C_{\text {out } H} \subset$ be the pullback,

$$
C_{\text {out } H}=C \cap L^{-1}\left(\left(\mathbb{R}_{+}^{n}\right)_{-\varepsilon}\right)
$$

for $\left(\mathbb{R}_{+}^{n}\right)_{-\varepsilon} \subset \mathbb{R}_{+}$being the half-space bounded by $\mathbb{R}_{-\varepsilon}^{n-1}$.

Observe that the 0-chain $H \cap C$ bound a 1-chain $C^{\prime} \subset U$, such that

- length $\left(C^{\prime}\right) \leq \lambda \operatorname{length}(C)$;

- $C^{\prime}$ is contained in the $d$-neighbourhood of $W$ for $d \leq \lambda \varepsilon$.

- the sum $C^{D}=C^{\prime}+C_{\text {out } H}$ of $C^{\prime}$ is an absolute cycle that bounds a 2-chain $D^{D} \subset U \cap \lambda \cdot B$ with

$$
\operatorname{area}\left(D^{D}\right) \leq 4 \lambda^{3} \cdot \text { length }(C)^{2},
$$

where $\lambda \cdot B$ denotes for a ball $B$ of radius $r$ denotes the concentric ball of radius $\lambda r$.

Thus, the inequality (1) is reduced to (2) with

a controlled "worsening" of the constants.

Now let us derive (2) on the $\rho$-scale from (1) on a significantly smaller scale $3 d$ as follows.

Let an $\operatorname{arc} C$ of length $l$ lie within distance $d$ from $W$. Subdivide $C$ into $k \leq(l+d) / d$ segments of length $\leq d$ and connect the ends of these segments with nearest points in $W$ by curves of length $\leq d$.

Thus, we decomposed the chain $C$ into the sum of $k$ chains $C_{i}, i=1,2, \ldots, k$ of length $\leq 3 d$ and summing up inequalities (1) for $C_{i}$ we obtain (2) for $C$.

Then we iterate this process with fillings of $C_{i}$ reduced to those for even smaller $C_{i j}$ etc.

Since the bound (2) in the filling volume (area for $k=2$ ) is significantly stronger then (1) for small $d$, the infinite iteration of this process produces a finite total sum of filling areas that satisfies the inequality (1). QED.

Remark. Even for smooth arks $C$, the final chain $D$ filling $C$ may became "infinitely complicated" in the vicinity of $W$. But if $W$ is smooth, then piecewise smooth $C$ will be filled by piecewise smooth surfaces $D$.

In order to apply a similar argument to higher dimensional cycles $k>2$ we observe the following.

(I) Cutting away the part of $C$ that is $d$-far from $W$ is same for all $k$ and causes no additional problem.

(II) Let $C \subset U$ be an absolute $(k-2)$-cycle, i.e. $\partial C=\varnothing$. If $\operatorname{Diam}(C) \leq \rho_{0}$ and $C$ lies within distance $d$ from $W=\partial U$, then $C$ bounds a relative $(k-1)$-chain $D$ such that

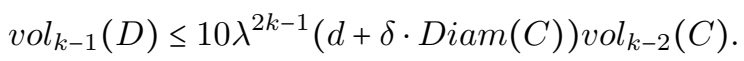

If $k=2$, this corresponds to moving ends of arks to $W$ where the Reifenberg's flatness of $W$ was unneeded. v

(III) A compilation for $k>2$ arises when we try to subdivide a chain $C$ of diameter $\rho$ that lies $d$-close to $W$ for $d<<\rho$ into pieces of diameters $\approx d$ and with $(k-1)$-volumes $\approx d^{k-1}$. This may be impossible if $C$ contains a significant thin part, where the intersections of $C$ with the balls $B_{c}(d) \subset X, c \in C$, have their volume much smaller than $d_{k-1}$. 
However, the inductive filling argument from [15] shows that the thin part can be filled in on the scale $\leq d$. Thus one may assume there is no thin part and $C$ can be subdivided into pieces of diameters about $d$, with $(k-1)$-volumes about $d^{k-1}$ and with $(k-2)$-volumes of the boundaries of these pieces about $d^{n-2}$. The latter together with (I) allows a decomposition $C=\sigma_{i} C_{i}$ as for $k=2$ and the validity of (1); hence of (2), disestablished for all $k$. QED.

Remark. The above argument makes only an rough outline of a proof. But the details are rather trivial if seen in the filling context of [15. In fact, the above argument perfectly works for general Banach spaces instead of $\mathbb{R}^{n}$ with (properly readjusted) constants depending on $k$ but not on $n$.

Corollary: $C^{0}$-Stability of Relative Bubbles. Let $X=\left(X, g_{0}\right)$ be a smooth Riemannian manifold, $U \subset X$ be a compact domain with smooth boundary $W=\partial U$ and let $Y \subset U$ be a smooth hypersurface with boundary in $W$ that is everywhere normal to $W$. Let $g_{\varepsilon}, \varepsilon>0$, be a family of smooth Riemannian metrics that $C^{0}$-converge to $g_{0}$ for $\varepsilon \rightarrow 0$.

Then there exist families of smooth domains $U_{\varepsilon}$ bounded by hypersurfaces $W_{\varepsilon}$ and of smooth hypersurfaces $Y_{\varepsilon} \subset U_{\varepsilon}$ with $\partial Y_{\varepsilon} \subset W_{\varepsilon}=\partial U_{\varepsilon}$ that are everywhere normal to $W_{\varepsilon}$ and where $W_{\varepsilon}$ converge to $W$ in the Hausdorff metric while $Y_{\varepsilon}$ Hausdorff converge to $Y$ for $\varepsilon \rightarrow 0$ and such that the mean curvatures of $W_{\varepsilon}$ and of $Y_{\varepsilon}$ converge to the mean curvatures of $W$ and $Y$ respectively.

Moreover, if $\varepsilon$ is sufficiently small, $\varepsilon \leq \varepsilon_{0}>0$, then the pair $\left(U_{\varepsilon}, Y_{\varepsilon}\right)$ can be joined with $(U, Y)$ be a diffeotopy in $X$ that is $C^{0}$-close to the identity diffeomorphism.

Half-Proof. The existence of $W_{\varepsilon}$ follows from the $C^{0}$-stability proven for individual manifolds in the previous section (where these were denoted $Y_{\varepsilon}$ ).

Then the same argument variation argument delivers $Y_{\varepsilon}$ with boundary in $W_{\varepsilon}$, since $W_{\varepsilon}$ is Reifenberg flat and satisfies the above filling inequality and this inequality does not allow escape of narrow fingers near $W_{\varepsilon}$.

It is known [26], [?] that such a $Y$ has the same type of regularity at the boundary as at the interior points. In particular, these $Y_{\varepsilon}$ are smooth up to the boundary if $n=\operatorname{dim} X \leq 6$.

One can not claim at this point that the manifolds $Y_{\varepsilon}$ are diffeomorphic to $Y$ for small $\varepsilon$ as it was done in the case of manifolds $Y$ without boundary, but the existence of such a diffeomorphism (and even of a diffeotopy) is ensured by the sharp filling inequality that we prove in the next section.

\subsection{Sharp Filling under Reifenberg's Controle.}

Let us generalize the definition of $(\delta, \lambda)$-flatness by replacing $\mathbb{R}^{n-1} \subset \mathbb{R}^{n}$ by a more general the hypersurface in $\mathbb{R}^{n}$.

We limit ourselves to the case where this hypersurface serves as the boundary of a compact convex domain $A \subset \mathbb{R}^{n}$ and define below $(\delta, \lambda)$-control of cooriented hypersurfaces $W \subset X$ by $\partial A$. Recall that coorientation means that we distinguished what is "locally inside" and what is "outside" $W$ and to simplify notation we assume that $W$ bounds a domain $U \subset X$.

Definition of $(\delta, \lambda)$-Control. A hypersurface $W=\partial U$ in Riemannian manifold $X \supset U$ is called $(\delta, \lambda)$-controlled by $\partial A \subset \mathbb{R}^{n}$ at a point $w \in W$ on the scale $\rho$, if there exists a $\lambda$-bi-Lipschitz "control" homeomorphism $L$ from the ball $B=B_{w}(\rho) \subset X$ to the Euclidean space $\mathbb{R}^{n}, n=\operatorname{dim}(X)$, such that 
- $L(U \cap B) \subset A$;

- the image $L(W \cap B) \subset \mathbb{R}^{n}$ is contained in the $\delta \rho$-neighbourhood of the boundary $\partial A \subset A$.

- the intersection of $\partial A$ with the Euclidean ball $B_{L(w)}\left(\lambda^{-1} \rho\right) \subset \mathbb{R}^{n}$ is contained in the $\delta \rho$-neighbourhood of the image $L(W \cap B) \subset A$.

We want to show that if $\lambda$ is close to 1 and $\delta$ is small, then the relative $k$-filling profile in $(U, \partial U)$ is almost the same as in $(A$,partial $A)$, where such a profile, $\operatorname{ReFill}_{A}^{k}(v), v>0$, is defined as the infimum of the filling volumes of the relative $(k-1)$-cycles $C$ in $(A, \partial A)$ with $v l_{k-1}(C) \leq v$. Namely we have the following

Sharp Filling Inequality. Let a hypersurface $W \partial U$ in Riemannian manifold $X \supset U$ be $(\delta, \lambda)$-controlled by $\partial A \subset \mathbb{R}^{n}$ at all point $w \in W$ and on all scales $\rho<\rho_{0}$.

Then the $k$-filling profiles of $U$ are bounded by those of $A$ as follows:

$$
\operatorname{ReFill}_{U}^{k}(v) \leq \alpha_{A}(\lambda, \delta) \operatorname{ReFill}_{A}^{k}(v) \text { for all } v \leq \operatorname{const}_{A} \lambda^{-k} \rho_{0}^{k-1}
$$

where $\alpha_{A}(\lambda, \delta) \rightarrow 1$ for $\lambda \rightarrow 1$ and $\delta \rightarrow 0$.

Proof. If a cycle $C$ is $d$-close to $W=\partial U$ for $d<v \operatorname{col}_{k-1}(C)^{1 /(k-1)}$, then the rough filling inequality $(2)$ from the previous section yields a much stronger filling bound than that by $\operatorname{ReFill}_{A}^{k}(v)$.

On the other hand, the part $C_{f a r} \subset C$ that is $d$-far from $W$ can be filled in $U$ as efficiently as in $A$. In fact, if $\lambda$ is close to 1 , one can actually think of this $C_{f a r}$ being the relative cycle in the subdomain $A_{-d} \subset A$ (that consists of the points that are $d$-far from the boundary $\partial A \subset A$ ) with $\partial C_{f a r}$ contained in the boundary of $A_{-d}$.

The only remaining problem is that the relative $k$-chain $D^{D} \subset A_{-d}$ that fills $\partial C_{f a r}$ modulo $\partial A_{-d}$ may have large $(k-1)$-volume of its intersection with $\partial A_{-d}$ that would make the $(k-1)$-volume of the cycle $C^{D}=\partial D^{D}$ much greater than that of $C$,

However, the coarea inequality, applied to $D^{D}$ intersected with the levels of the distance function $a \mapsto \operatorname{dist}(a, \partial A)$, shows that some of these intersections for possibly larger but still controllably small $d^{\prime} \geq d$ will have their volumes not much larger than that of $C$ unless all of $D^{D}$; hence, all of the $C$, lie within distance $<<\operatorname{vol}_{k-1}(C)^{1 /(k-1)}$ from the boundary $\partial A$. This yields the proof of the sharp inequality that completes the proof of the above $C^{0}$-stability of relative bubbles.

Remarks. (a) We formulated both, rough and sharp filling inequalities for a specific purpose of proving the $C^{0}$-stability of the mean curvatures \& dihedral angles of hypersurfaces. Probably, there is a more general formulation of such an inequality and a more transparent proof that is not overburdened with trivial technicalities.

(b) If $\delta>0$ is sufficiently small $\delta \leq \delta_{0}(A, \lambda)>0$, and $W \subset X$ is $(\delta, \lambda)$ controlled by $\partial A \subset \mathbb{R}^{n}$, then, apparently, $W$ is a Höder submanifold in $X$. This seems to follow by the argument from [43] and/or [9].

\subsection{Reifenberg and Höder at the Corners.}

Let us recall relative bubbles $Y_{\varepsilon} \subset U_{\varepsilon}$ with their boundaries $\partial Y_{\varepsilon} \subset W_{\varepsilon}=\partial U_{\varepsilon}$ in Riemannian manifolds $X=\left(X, g_{\varepsilon}\right)$ with smooth Riemannian metrics that 
$C^{0}$-approximate the original metric $g_{0}$ on $X$.

Let $U_{\varepsilon 1} \subset U$ be a cornered subdomain bounded in $U_{\varepsilon}$ by $Y_{\varepsilon}$ and let $A\left\ulcorner\subset \mathbb{R}^{n}\right.$ be the intersection of two half spaces bounded by mutually orthogonal hyperplanes, i.e. $A\left\ulcorner=\mathbb{R}_{+}^{2} \times \mathbb{R}^{n-2}\right.$.

Observe that the evaluation of the filling profile of $A\ulcorner$ reduces to that for $\mathbb{R}^{n} \supset A\left\ulcorner\right.$, since every relative cycles $C \subset A\left\ulcorner\right.$ define an absolute cycle in $\mathbb{R}^{n}$, call $4\ulcorner C$ that is obtained by reflecting $C$ four times around the $(n-1)$-faces of $A\ulcorner$. Thus, the extremal relative $(n-2)$-cycles $C$ (we care for the sharp inequality only for $k=n-1)$ i.e. those maximizing

$$
\frac{\operatorname{ReFill}^{k}(C)^{\frac{1}{k}}}{\operatorname{vol}_{k-1}(C)^{\frac{1}{k-1}}}
$$

are intersections of $A\ulcorner$ with the round (n-2)-spheres that meet the two $(n-1)$ faces of $A\left\ulcorner\right.$ at the $90^{\circ}$ angles.

The limit argument that was used in section 4.6 for deriving the Reifenberg flatness of $Y_{\varepsilon \rightarrow 0}$ implies the following.

There exist functions $\lambda=\lambda(\rho, \varepsilon)>1$ and $\delta=\delta(\rho, \varepsilon)>0$, such that $\lambda(\rho, \varepsilon) \rightarrow 1$ and $\delta(\rho, \varepsilon) \rightarrow 0$ for $\rho \rightarrow 0$ and $\varepsilon \rightarrow 0$ and such that the boundaries of the domains $U_{\varepsilon 1}$ are $(\lambda, \delta)$-controlled by $A\left\ulcorner\right.$ at the scales $\leq \rho$ and at all points $x \in \partial U_{1}$. .

It follows that the domain $A\ulcorner$ has almost the same filling profile on the small scales $\rho$ as $A\left\ulcorner\right.$. Therefore, one is in a position to construct bubbles in $U_{\varepsilon 1}$, call them $Y_{\varepsilon 1} \subset U_{\varepsilon 1}$, with respect to the metric $g \varepsilon$ with boundaries in $\partial Y_{\varepsilon 1} \subset \partial U_{\varepsilon 1}$ with the same efficiency and the same properties as we did it earlier in $U_{\varepsilon}$, EXCEPT that we can not guarantee the smoothness of $Y_{\varepsilon 1}$ at the points where $Y_{\varepsilon 1}$ meets the corner of $U_{\varepsilon 1}$.

However, all of the above seem to apply to general to general $\mu$-bubblehedra and yield the following solution of the Hölder-regular mean curvature $C^{0}$-stability problem.

Let $P$ be a compact strictly preconvex cosimplicial polyhedral domain in a smooth Riemannian manifold $X=(X, g)$.

(Recall that "strictly preconvex" signifies the bound $<_{i j}<\pi$ for the dihedral angles of $P$; one distinguishes the case $<_{i j}=\pi / 2$ where the " $\mu$ " in " $\mu$-bubble" are measures given by continuos density functions.

Also notice that an arbitrary $P$ becomes cosimplicial, as defined in section 1 under a generic perturbation of its faces and the general case can be probably reduced to the cosimplicial one.)

Let $X^{\prime}$ be another Riemannian manifold with a family of $C^{2}$-smooth metric $g_{\varepsilon}^{\prime}$ and let $f_{\varepsilon}: X^{\prime} \rightarrow X$ be an $e^{\varepsilon}$ - bi-Lipschitz homeomorphisms.

Then there exist $C^{\alpha}$-bi-Hölder homeomorphisms $f_{\varepsilon}^{\prime}: X^{\prime} \rightarrow X$ for some $\alpha>0$ with the following properties.

- $\rightarrow 0$ The maps $f_{\varepsilon}^{\prime}$ converge to $f_{\varepsilon}$ in the $C^{0}$-topology for $\varepsilon \rightarrow 0$.

- reg the maps $f_{\varepsilon}^{\prime}$ are $C^{\infty}$ diffeomorphisms away from the corners, i.e. $(n-3)$ faces, $n=\operatorname{dim} X$, of $P_{\varepsilon}^{\prime}=\left(f_{\varepsilon}^{\prime}\right)^{-1}(P) \subset X^{\prime}$.

-stbl

$$
\left|m n . \operatorname{curv}_{x^{\prime}}\left(P_{\varepsilon}^{\prime}\right)-m n . \operatorname{curv}_{x}(P)\right| \leq \kappa(\varepsilon) \rightarrow 0 \text { for } \varepsilon \rightarrow 0,
$$

for all $x^{\prime}$ away from the corners of $P_{\varepsilon}^{\prime}$. 
(Recall that the mean curvature at the edges, i.e. $(n-2)$-faces, $n=\operatorname{dim} X$, is defined in terms of the dihedral angles as $\pi-\angle_{i j}$.)

We do not go into the detailed proof since this result is neither general enough to elucidate the geometric meaning of scal $\geq 0$ nor is the Hölder regularity is sufficient for our applications to positive scalar curvature.

On the other hand, this insufficient regularity can be bypassed in the $C^{0}$ non-approximation application as we shall see below.

\subsection{Proof of the $C^{0}$-Limit Theorem.}

We shall show in this section that

Smooth metrics $g$ of negative scalar curvature can not be $C^{0}$-approximated by metrics $g_{\varepsilon}$ with nonnegative scalar curvatures.

Proof. Let $g$ me a smooth metric on an $n$-manifold $X$ with negative scalar curvature at a point $x_{0} \in X$. Then

(口) all sufficiently small neighbourhoods of $x_{0}$ contain (tiny) mean curvature convex cubical polyhedral domains $P \ni x_{0}$ with strictly acute (i.e. $<\pi / 2$ ) dihedral angles.

Proof of (ם) . We assume by induction that such domains exist in submanifolds $X^{\prime} \subset X$ that contain $x_{0}$ that that have zero second fundamental form at $x_{0}$.

Clearly, there exists a codimension 1 submanifold in $X^{\prime} \subset X$ that contains $x_{0}$, such that

(•) submanifold $X^{\prime}$ is totally geodesic at $x_{0}$ i.e. its second fundamental form vanishes at $x_{0}$;

(•) the induced metric in $X^{\prime}$ has strictly negative scalar curvature;

$(\bullet)$ the Ricci curvature of $X$ at the normal vector $\nu_{0}$ to $X^{\prime}$ at $x_{0}$ is strictly negative.

It is also clear that such an $X^{\prime}$ admits an arbitrary small perturbation, call it $X^{\prime \prime} \subset X$ near $x_{0}$ that still contains $x_{0}$ and such that its mean curvature becomes zero near $x_{0}$ while keeping the second fundamental form II zero at $x_{0}$. (Small non-zero II will also do.)

We may assume by induction on $\operatorname{dim}(X)$ that $X^{\prime \prime}$ contains required polyhedral domains, say $P^{\prime \prime} \subset X^{\prime}$. Take such a $P^{\prime \prime} \ni x_{0}$ in $X^{\prime \prime}$ of very small diameter $\delta$ and let $P=P^{\prime \prime} \times[-\varepsilon,+\varepsilon]$ be the union of the geodesic $2 \varepsilon$-segments normal to $P^{\prime \prime}$ and going by $\varepsilon$ in both normal directions at all points in $P^{\prime \prime} \ni x_{0}$.

If $\varepsilon$ is sufficiently small that the "horizontal" faces $P^{\prime \prime} \times \pm \varepsilon \subset X$ have positive mean curvatures by the second variation formula. It is also clear that of the dihedral angles between these horizontal faces and the remaining "vertical" ones equal $\pi / 2$ while the angles between vertical angles are $<\pi / 2$ for small $\varepsilon$. Also a simple computation shows that the mean curvatures of the vertical faces are positive.

Thus $P$ satisfies all requirements except for having some dihedral angles $=\pi / 2$ but these can be made acute by an arbitrary small perturbations of the two "horizontal" faces.

If we could prove the $C^{0}$-stability of the mean convexity for this $P$ with $P_{\varepsilon}^{\prime}=\left(f_{\varepsilon}^{\prime}\right)^{-1}(P)$ being $C^{2}$-smooth (rather than mere Hölder as in the previous section) everywhere including the corners, then we would apply "gluing around 
the edges" to $P_{\varepsilon}^{\prime}$ as in section 2.1 and arrive at a metric with positive scalar curvatures on the $n$-torus. But as we have not proved this stability, we need to combine the mean curvature stability for individual hypersurfaces (i.e. polyhedral domains of depth $d=1$ ) with the gluing as follows.

Let $X=(X, g)$ be a smooth Riemannian manifold with compact strictly mean curvature convex boundary $Y=\partial X$. If $\operatorname{scal}(g)>0$ then (see [23, [2]) the double $2 \underset{Y}{\diamond} X$ admits a family of metrics, say $2 \diamond g_{\delta}, \delta>0$, where $g_{\delta}$ are metrics on $X$ such that:

$\bullet \leq \delta$ the metrics $g_{\delta}$ are $\delta$-close to $g$ in the $C^{0}$-topology;

- $C^{2}$ the double metrics $2 \diamond g_{\delta}$, the restriction of which on both copies of $X \subset 2 \underset{Y}{\diamond} X$ by definition equal $g_{\delta}$, are $C^{2}$-smooth;

(Notice, that the $C^{1}$-smoothness of $2 \diamond g_{\delta}$ is equivalent to $Y$ being totally geodesic with respect to $g_{\delta}$, and $C^{2}$-says something about the curvature tensor of $g_{\delta}$ on $Y=\partial X$.)

- $s c>0$ the metrics $g_{\delta}$, and hence $2 \diamond g_{\delta}$, have positive scalar curvatures.

Let $P$ be an $n$-dimensional rectangular reflection domain (see section 2 ), e.g. a cubical one, and let $g$ be a smooth Riemannian metric on $P$ with respect to which the faces of $P$ have strictly positive mean curvatures and the dihedral angles are all $\pi / 2$.

Let $g_{\varepsilon}$ be a family of smooth Riemannian metrics on $P$ such that:

- $\leq \varepsilon$ the metric $g_{\varepsilon}$ is $\varepsilon$-close to $g$, in the $C^{0}$-topology for all $\varepsilon<0$;

- $s c \geq 0 \operatorname{scal}\left(g_{\varepsilon}\right) \geq 0$ all $\varepsilon<0$.

Approximation/Reflection Lemma. There exists metrics $g_{\varepsilon, \delta}$ on $P$ for all $\varepsilon, \delta>0$, and polyhedral subdomains $P^{\prime}=P_{\varepsilon, \delta}^{\prime} \subset P$ such that

- the domains $P^{\prime}$ are combinatorial equivalent to $P$, where such an equivalence is established by homeomorphisms $P \rightarrow P^{\prime}$ that $C^{0}$-converge to the identity map for $\varepsilon, \delta \rightarrow 0$;

$\bullet_{\varepsilon+\delta}$. The metric $g_{\varepsilon, \delta}$ is $\delta$-close to $g_{\varepsilon}$; thus, it is $(\varepsilon+\delta)$-close to $g$, for all $\varepsilon, \delta>0$

- $s c \geq 0 \operatorname{scal}\left(g_{\varepsilon, \delta}\right) \geq 0$ for all $\varepsilon, \delta>0$;

- reg all dihedral angles of $P^{\prime}$ with respect to $g_{\varepsilon, \delta}$ equal $\pi / 2$ and all faces of $P^{\prime}$ are totally geodesic; moreover the canonical extension of $g_{\varepsilon, \delta}$ to the metrics $\tilde{g}_{\varepsilon, \delta}$ on the manifold $\tilde{P}^{\prime} \supset P^{\prime}$ where the corresponding reflection group $\Gamma$ acts are $C^{2}$-smooth for all $\varepsilon, \delta>0$.

Proof. Proceed by modifying faces of $P$ one by one and simultaneously changing the metric.

Namely, assume by induction that $\bullet_{\text {reg }}$ is satisfied by some faces, call them $W_{\text {reg }}^{\prime}$ and dihedral angles between them for some metric $g_{r e g}$. Then "regularize" an extra face, say $W_{i}$ by solving the corresponding Plateau $\mu$-bubble problem (see the previous section) where the boundary of the new face $W_{i}^{\prime}$ - solution of this Plateau problem, is contained in the union of the faces $W_{\text {reg }}$ and of all not yet regularized faces $W_{j}$ except for $W_{i}$ itself.

Observe that

the regularity of the extremal $W_{i}^{\prime}$ at the points in the union of $W_{\text {reg }}$ follows from the interior regularity by the standard and obvious reflection argument. 
Finally, replace $g_{r e g}$ by the above $g_{r e g, \delta}$ that makes $W_{i}^{\prime}$ regular as well; thus, the inductive step is accomplished.

An essential point here is that the condition $\bullet \leq \delta$ implies that the metric $g_{\text {reg, } \delta}$ may be assumed arbitrarily $C^{0}$ close to $g_{r e g}$ and so the relevant filling profiles essentially do not change as we pass from $g_{r e g}$ to $g_{r e g, \delta}$; thus, the process of consecutive "regularization" of faces and metrics goes unobstructed.

Warning. The metrics $g_{\varepsilon, \delta}$ are $C^{0}$-close to $g$ but, in general, this closeness, unlike that between $g_{r e g}$ and $g_{r e g, \delta}$, "does not respect" the boundary $Y=\cup_{i} W_{i}$ of $P$ : there is no Lipschitz control over the homeomorphism that moves $P$ to $P^{\prime}$.

Conclusion of the Proof of the $C^{0}$-Limit Theorem. The cubical domain from the above ( $\square$ ) can be trivially made strictly mean convex with all dihedral angles $\pi / 2$ and, by the Lemma, the the solution of the Geroch conjecture for tori applies.

This settles the case $\kappa=0$ that extends to $\kappa \neq 0$ by (a) of 1.8 .

\section{Conjectures and Problems.}

\subsection{On Topography of Plateau Wells.}

Existence/non-existence of positive scalar curvature on a manifold $X$ is invariant under the codimension 2-surgery of $X$, i.e. adding $m$-handles with $m \leq n-$ 2 ; accordingly, one wishes to have a counterpart of the $C^{0}$-non-approximation property in the category of manifolds taken modulo such surgery whatever this means.

This agrees with the observation that attaching "thin $m$-handles" to $X$ with $m \leq n-2$ does not significantly change the topography of Plateau traps in $X$ that can be seen as "wells" in the $(n-1)$-volume landscape in the space of hypersurfaces in $X$.

A representative example of such "insignificant" change is as follows.

Let $Y \subset X$ be a locally $v l_{n-1}$-minimizing hypersurface which is trapped in a small neighbourhood $U \supset Y$.

Let $C$ be a smooth curve joining two points $x_{1}, x_{2} \in X \backslash U$ which are positioned "relatively far from" $U$ and such that $C$ itself as well as all "moderately large" perturbations" $C^{\prime}$ of $C$ intersect $Y$.

Take an $\varepsilon$-thin normal neighbourhood $D \supset C$ and modify the original metric $g$ of $X$ on $D$ by enlarging the lengths of the geodesic segments in $D$ normal to $C$ by the factor $\varepsilon^{-1-\alpha}$, for a small $\alpha>0$, e.g. $\alpha=0.1$.

If $n=\operatorname{dim}(X) \geq 3$, then the resulting enlarged metric $g^{\prime} \geq g$ on $X$ is, "on the average", $\varepsilon^{\beta}$-close to $g$ for $\beta>0$ and, in many cases, there is a minimal $Y^{\prime} \subset X^{\prime}$ corresponding to $Y$, which, for $n-1=\operatorname{dim}(Y) \geq 2$, is "on the average" $\approx \varepsilon^{\gamma}$-close to $Y$ for a $\gamma>0$.

But since such a $Y^{\prime}$ must intersect $C$, it is obtained from (a slightly perturbed) $Y$ by attaching several thin and $\approx \varepsilon^{-\alpha}$-long "fingers" corresponding to (sufficiently stable) intersection points of $C$ with $Y$. Since length fing $\rightarrow \infty$ for $\varepsilon \rightarrow 0$ one can not avoid "on the average".

Similarly, one can compare traps in non-equidimensional manifolds. The simplest examples are Riemannian product manifolds, where, e.g. $X^{\prime}=X \times \mathbb{R}^{k}$ 
has essentially the same minimal subvarieties and Plateau wells as $X$.

Besides looking at what happens to $X^{\prime}$ in the "immediate neighbourhood" of an $X$ we want to keep track of traps/wells in manifolds $X^{\prime}$ that are only moderately close to $X$ and where only relatively deep/wide Plateau wells in $X$ have a chance of being shadowed by wells in $X^{\prime}$.

\subsection{Webs and Honeycombs.}

A Plateau $m$-web $\mathcal{M}$ in a Riemannian $n$-manifold $X$ is an $m$-tuple of foliations $\mathcal{M}_{i}, i=1,2, \ldots m$ by minimal subvarieties of codimension 1 such that the subvarieties from different foliations are mutually transversal and make constant (dihedral) angles, say $\angle_{i j}, i, j=1,2, \ldots m$.

Local Web Conjecture. If $m>n$ then such a web is locally isometric to the flat one, i.e. to $\mathbb{R}^{n}$ with $m$ families of parallel hyperplanes. Furthermore, if $m=n$ and the web is normal, i.e. $\angle_{i j}=\pi / 2$ for all $j \neq i=1,2, \ldots n$, then it is also flat, provided $X$ has $S c \geq 0$.

Let us look closer at the normal webs.

First, every transversal $n$-web locally equals a coordinate web: there are local coordinates $x_{1}, \ldots, x_{n}$ in $X$, where $\mathcal{M}$ identifies with the $n$ families of the coordinate hypersurfaces $x_{i}=$ cost .

The normality condition signifies that the $g_{i j}$-terms of the Riemannian metric vanish for $i \neq j$, while the minimality implies that the products $G_{i}=\prod_{j \neq i} g_{j j}$ are invariant under the flows by the (coordinate) vector fields $\partial_{i}=\partial / d x_{i}$.

It follows, that each $G_{i}$ is, in fact, a (positive) function in $n-1$ (rather than $n$ ) variables, namely in $x_{j}$ for $j \neq i$, and every $n$-tuple of such functions defines a normal Plateau web, where $g_{i i}$ are uniquely determined by the equations $\prod_{j \neq i} g_{j j}=G_{i}$.

It is seems not hard to show by a direct computation that the scalar curvature of such metric $\sum_{i} g_{i i} d x_{i}^{2}$ is strictly negative unless the web is flat, but I did not hat this. On the other hand the inequality $s c a l \leq 0$ follows, as we know, from the Geroch conjecture for tori.

Apparently, the above representation of metrics generalizes to non-normal Plateau $n$-webs (with constant but not normal $\angle_{i j}$ ); this would imply the local conjecture for $(m>n)$-webs.

Let us generalize the concept of normal web as follows.

A cubical polyhedron $P_{0}$ is called a normal Plateau honeycomb if a Riemannian $n$-manifold $X$ is a collection $\mathcal{P}$ of cubical domain $P \subset X$ such that $P_{0} \in \mathcal{P}$ and

- the faces of all $P \in \mathcal{P}$ are minimal (possibly singular?) hypersurfaces in $X$ where all dihedral angles between the faces equal $\pi / 2$;

- there exists a face preserving continuous map of every $P \in \mathcal{P}$ onto the $n$-cube $[0,1]^{n}$ such that the pullbacks of all $(n-1)$-subcubes in it parallel to the faces, $[0,1] \times[0,1] \times \ldots \times t \times \ldots \times[0,1], t \in[0,1]$ are minimal hupersurfaces, call them $Q \subset P$ normal to the boundary of $P$;

- the two parts into which such a $Q$ divides $P$ are cubical domains that are elements of $\mathcal{P}$. 
Motivation. Let $P_{0}$ be a non strictly mean curvature convex cubical polyhedral domain, say $P_{+} \subset P$, with all dihedral angles $\leq \pi / 2$. Then either $P_{0}$ contains a strictly mean curvature convex cubical polyhedral domain with all dihedral angles $\leq \pi / 2$ or $P_{0}$ is a normal Plateau honeycomb.

Justification. If there is a regular point in a face where the mean curvature is strictly positive, or if some angle $\angle_{i j}$ is somewhere, $\pi / 2$, then $P^{\prime}$ can be obtained by smoothing of an arbitrary small perturbation of $P$ which is achieved by an elementary linear(ization) argument.

Thus, we may assume that $P$ is normal Plateau. If one of faces, say $Y$ of $P$ is not locally minimizing, one can cut $P$ by a minimizing face and thus one may assume that every pair of opposite faces in $P$ has at least one of them, say $Y$, being locally minimizing.

If such a $Y \subset P$ is not isolated, it serves as a leaf of a Plateau foliation and if assume non-existence of a Plateau honeycomb, we conclude that some of these faces, let it be $Y$, is isolated.

Then, for a sufficiently small $\varepsilon$, there exists an $\varepsilon$ bubble $Y_{\varepsilon} \subset P$ and then the "band" $P^{\prime}$ between this $Y_{\varepsilon}$ and $Y$ in $P$ does the job (after a small perturbation and smoothing making all faces of $P^{\prime}$ strictly mean convex).

Remark. A technical difficulty in this argument resides in the regularity of our bubbles, especially at the corners.

Questions. Do all normal Plateau honeycombs are isometric to Euclidean solids?

Does it help to assume that $\operatorname{scal}(P)=0$ ?

How much does a presence of singularities in minimal hypersurfaces $Q$ complicate the geometry of $P$ ?

\subsection{Nested Cubes and Small Diameter Conjecture.}

Let $P \subset X$ be a normal (i.e. with mutually normal faces) mean curvature convex cubical polyhedral domain of depth $d=n=\operatorname{dim}(X)$ in a Riemannian manifold $X$.

Does $P$ contains a normal $n$-cubical subdomain $P_{\square} \subset P$ of an arbitrary small $\operatorname{diam}_{X}\left(P_{\square}\right) \leq \delta$ for a given $\delta>0$.

Notice that according to () from section 4.9 a presence of a point in $P$ where scalar curvature $<0$ implies the existence of $P_{\square}$ and the solution of the $P_{\square}-$ problem follows in some cases from the solution of the Geroch conjecture, e.g. where the faces of $P$ are smooth with strictly positive mean curvatures.

But it is instructive to construct $P_{\square}$ by a direct argument keeping an eye on singular spaces, where the simplest case is that of a manifold $X$ with a $C^{1}$ smooth metric where curvature is defined only in distribution sense. Moreover, one can formulate this problem even for $C^{0}$-metrics in terms of $\Delta$-stable mean convexity (see section 4.4) where no curvature exists even in a weak sense.

More specifically, say that a cubical subdomain $P^{\prime} \subset P$ is a sandwich in $P$ if, combinatorially, $P^{\prime}$ looks as a "rectangular slice" of a cube, namely as $[a, b] \times[0,1]^{n-1} \subset[0,1]^{n}$ for $0 \leq a<b \leq 1$.

In other words, $P^{\prime}$ is bounded in $P$ by a pair of its mutually disjoint "new" faces that are hypersurfaces, say $Q_{(}^{\prime}$ and $Q^{\prime}$ in $P$, both separating a pair of opposite faces, say $Q_{(}$and $Q_{\text {) }}$ of $P$, where "separating" here also signifies "being homologous to" in the the obvious sense. Thus, the boundaries $\partial Q^{\prime}$ and $\partial Q_{-}^{\prime}$ 
are contained in the boundary $\partial P$ where they intersect all faces, but $Q$ and $Q_{-}$ of $P$, unless one (or both) of these new faces equals the "old" $Q$ or (and) $Q_{-}$.

Consider decreasing sequences $P=P_{0} \supset P_{1} \supset P_{2} \supset \ldots \supset P_{k} \ldots$ of normal cubical domains where $P_{k}$ is a sandwich in $P_{k-1}$ for all $k=1,2, \ldots$.

Call a closed subset $P_{\infty} \subset P$ a normal micro-cube if it equals the intersection of $P_{k}$ in such a sequence.

Example. If $P$ equals the ordinary cube $[0,1]^{n}$ then (connected) micro-cubes are exactly Cartesian products of $n$ subintervals in $[0,1]$, possibly, some reduced to points.

Question. What are geometries and topologies of these micro-cubes?

Conjecture. Every normal cubical domain $P$ of depth $d=n=\operatorname{dim} X$ contains a zero dimensional micro-cube in it.

If $Y$ and, hence $Y_{\varepsilon}$ are regular, then such band can be regarded as an "infinitesimally thickened" $(n-1)$ dimensional cubical polyhedron and, after $n$ steps, one arrives at a zero dimensional "cube" $P_{\square} \subset P$.

But this dimension reduction process does not a priori apply to quasiconical singularities where the dimension reduction is not apparent. (We temporarily disregard singularities at the corners at this point.)

Thus all we can claim is that some $P_{\square}$ is no greater than the singularity; thus, $\operatorname{dim}\left(P_{\square}\right) \leq n-8$.

Then, if $\operatorname{dim} X \leq 9$, some cubical domain $P_{k}$ approximating $P_{\square}$ is spin, since the the 2-dimensional Stiefel-Whitney class $w_{2} \in H^{2}\left(P ; \mathbb{Z}_{2}\right)$ vanishes on $P_{\square}$ that have dimension $<2$ and the Dirac operator method applies.

Corners do not Matter. Indeed,we can arrange $P_{k}$ such that $P_{k+n}$ is contained in the interior of $P_{k}$ for all $k$. Thus, any nuisance at the boundary will be eventually forgotten. Besides we can apply arbitrarily small $C^{0}$-perturbations to the Riemannian metrics $g_{k}$ in $P_{k}$, that smoothes the natural extensions $\tilde{g}_{k}$ of of $g_{k}$ to the orbicoverings (reflection developments) $\tilde{P}_{k}$ of $P_{k}$ as in section 2 .

Further Remarks and Questions. (a) There is counterpart of the small diameter problem that appeals to "billiard minimal" hypersurfaces that do not have to be "parallel" to the original faces of $P$ and that can be formulated in terms of the $\mathbb{T}^{n}$-essential manifold $X=\tilde{P} / \mathbb{Z}^{n}$ associated to $P$ as follows.

Take a minimal $(n-1)$-cycle $Y(1) \subset X(1)=X$ which may be different from those corresponding to faces of $P$. Then take an infinitesimally narrow "bubble-band" $X^{\prime}(1) \subset X(1)$ around $Y(1)$ and that is bounded by two smooth hypersurfaces with positive mean curvatures as we did in section 4.3. Let $X(2)=$ $2 X^{\prime}(1)$ be the double of $X^{\prime}(1)$.

Continue similarly with some $Y(2) \subset X(2)$ thus obtaining $X(3)$, etc.

$X(1), X(2), X(3), \ldots$

Do the diameters of $X(i)$ (as well as of the Plateau-hedra obtained by "cutting these $X(i)$ open") converge to zero for $i \rightarrow \infty$ for suitable $X(i)$ ?

(b) Can one move several faces of a $P$ inward simultaneously, e.g. near a corner (vertex) of $P$ by solving the corresponding linearized equations and applying an implicit function theorem?

(c) Let $X$ be an dimensional Riemannian manifold and $Z \subset X$ be a compact piecewise smooth submanifold of dimension $\leq n-2$, e.g a curve in $\mathbb{R}^{3}$. 
Suppose that $Z$ admits an a mean curvature convex normal cubical approximation i.e. it equals the intersection of a decreasing family of normal $n$-cubical m.c.c. polyhedra $P(k) \subset X, k=1,2, \ldots$.

Is then $Z$ necessarily smooth away from its boundary? Is it, moreover, totally geodesic?

We are especially concerned with the possibility of a "bad" (but, potentially most interesting) approximation, where all faces $W(k)=W^{n-1}(k)$ of $P(i)$ (there are $2 n$ of these faces) and, consequently, the $n-2$-faces, are eventually dense in $Z$, i.e. $\operatorname{dist}_{\mathrm{Hau}}(Y(i), Z) \rightarrow 0$, for $i \rightarrow \infty$ and for every sequence of $(n-1)$-faces $Y(i)$ of $P(i)$.

Does a $Z$ which admits a "bad" approximation by normal cubical m.c.c. (or Plateau) $P$ necessarily consist of a single point?

This seems easy for $n \leq 7$ but a similar question for non-cubical $P$ appears non-trivial even for mean curvature convex $P \subset \mathbb{R}^{3}$.

(The worst scenario from the perspective of the $s c a l>0$-problems is when not only the families of faces $W(i)$ of $X(i)$ are eventually dense in $Z$, but every family of stable minimal "billiard subvarieties" $Y^{\prime}(i) \subset X(i)$ is also eventually dense in $Z$.

(d) Possible quasi-conical singularities of minimal varieties is the apparent source of our problems, but, eventually, singularities should serve in our favor: they significantly constrain the shape of minimal varieties.

For example, let $X$ be a compact Riemannian manifold. Then, probably, every "small" cubical Plateau-hedron $P \subset X$ with $\angle_{i j} \leq \pi / 2$ and with locally minimizing $(n-1)$-faces has all these faces non-singular, say in the interior points, where "small" means $\operatorname{diam}(P) \leq$ const $_{X}$.

(e) Observe the following converse to the existence of mean convex neighbourhoods of isolated minimizing hypersurfaces.

Let a connected Riemannian $n$-manifold $X$ be divided by be a compact connected subset $Y$ in into two connected domains $X_{+}$and $X_{-}$with common boundary $\partial X_{+}=\partial X_{-}=Y$.

Let $Y_{0}$ equals the intersection of compact domains $W \supset Y_{0}$ in $X$ with smooth mean curvature convex boundaries in $X$.

If $Y$ has finite $(n-1)$-dimensional Hausdorff measure, vol $_{n-1}(Y)<\infty$, then $Y$ is locally (in the space of $\mathbb{Z}$-currents) vol ${ }_{n-1}$-minimizing. In particular, $Y$ is smooth of dimension $n-1$ away from a compact subset $\Sigma \subset Y_{0}$ with $\operatorname{dim}_{H a u}(\Sigma) \leq$ $n-8$.

(I am uncertain on what happens if $v o l_{n-1}(Y)=\infty$ that pertains to the question of possible topologies of Hausdorff limits of stable minimal hypersurfaces with volumes $\rightarrow \infty$ in $X$.)

(f) Let $Z=\cap_{k} P(k)$ for a sequence of m.c.c. polyhedra $P(k)$ of same combinatorial types and with given bounds in their dihedral angles, where one distinguishes the case where all $P(k)$ are Plateau-hedra.

Consider ("informative") sequences of points $x_{i} \subset X$ and sequences of numbers $\lambda_{i} \rightarrow \infty$, and take the (sub)limits of $\lambda_{i} P(i) \subset \lambda_{i}\left(X, x_{i}\right)$.

How much of geometry of $Z$, e.g. in the case of "bad approximation", can be extracted from the resulting Euclidean picture(s)? 


\subsection{Gauss Bonnet Prism Inequalities and the Extremal Model Problem.}

Let $P$ be a mean curvature convex 3 -dimensional Riemannian manifold with corners that is combinatorially equivalent to a prism, that is a product of a $k$-gon by a line segment.

Let the dihedral angles at the top and at the bottom of $P$ be $\leq \pi / 2$, call such prisms normal, and let the dihedral angles between the remaining faces (sides) of $P$ be bounded by some numbers $\alpha_{1}, \ldots, \alpha_{i}, \ldots, \alpha_{k}$.

$3 D$ Gauss Bonnet Prism Inequality. If $P$ has non-negative scalar curvature, then the numbers $\alpha_{i}$ are bounded from below by

$$
\sum_{i=1, \ldots, k}\left(\pi-\alpha_{i}\right) \leq 2 \pi
$$

Proof. Let $Y_{\min } \subset P$ be an area minimizing surface separating the top of $P$ from the bottom, that, observe, is normal to $\partial P$.

Temporally assume that $Y_{\min }$ is $C^{2}$-smooth including the corner points where $Y_{\min }$ meets the "vertical" edges of $P$ and recall that the second variation of $\operatorname{area}\left(Y_{\min }\right)$ is

$$
\operatorname{area}^{\prime \prime}=-\int_{Y_{\min }} \operatorname{Ricci}_{P}(\nu) d y-\int_{\partial Y_{\min }} \operatorname{curv}_{\nu}(\partial P) d s
$$

for $s$ being the length parameter in $\partial Y_{\min }$.

Observe following 45] (also compare 8]) that

$$
\operatorname{Ricci}_{P}(\nu)=\frac{1}{2}\left(\operatorname{scal}(P)+\lambda_{1}^{2}+\lambda_{2}^{2}\right)-K\left(Y_{\text {min }}\right)
$$

for $\lambda_{i}$ denoting the principal curvatures of $Y_{\min }$ and $K$ being the sectional curvature of $Y_{\min }$, while

$$
\operatorname{curv}_{\nu}(\partial P)=m n . \operatorname{curv}(\partial P)-\operatorname{curv}\left(\partial Y_{\min }\right) .
$$

Thus, we conclude as Schoen and Yau do in 45] that

$$
0 \leq \operatorname{area}^{\prime \prime} \leq \int_{Y_{\min }} K\left(Y_{\min }\right) d y+\int_{\partial Y_{\min }} \operatorname{curv}\left(\partial Y_{\min }\right) d s,
$$

where

$$
\int_{Y_{\min }} K(Y) d y+\int_{\partial Y_{\min }} \operatorname{curv}\left(\partial Y_{\min }\right) d s+\sum_{i}\left(\pi-\alpha_{i}\right)=2 \pi \chi\left(Y_{\min }\right)
$$

by the Gauss Bonnet formula. Hence,

$$
\sum_{i}\left(\pi-\alpha_{i}\right) \leq 2 \pi \chi\left(Y_{\min }\right) \leq 2 \pi
$$

On Regularity at the Corners. The above $C^{2}$-smoothness assumption may be violated at the corners of $Y_{\min }$ but it is easy to see by looking at how $Y_{\min }$ is approximated at the vertices by the necessarily unique tangent cones, that $Y_{\text {min }}$ admits tangent planes (or rather cones) at the corners. 
Also one sees in this limit cone picture that the length $\left(\partial Y_{\min }\right)<\infty$; hence $\int_{\partial Y_{\min }}\left|\operatorname{curv}\left(\partial Y_{\min }\right)\right| d s<\infty$ and since by minimality of $Y_{\min }$ the curvature $K(V)$ is bounded from above, $\int_{Y_{\min }}\left|K\left(Y_{\min }\right)\right| d y<\infty$. This is sufficient to justify the above computation.

Extremality and Rigidity. The above argument shows that convex Euclidean prisms are extremal for the prism inequality, moreover they are dihedrally rigid:

If a normal mean curvature convex prism $P$ with $\operatorname{scal}(P) \geq 0$ satisfies $\sum_{i}(\pi-$ $\left.\alpha_{i}\right)=2 \pi$ then it is isometric to a convex Euclidean prism.

Non-zero Bounds on Scalar Curvature. If $\operatorname{scal}(P) \geq 2 \kappa$ then the inequality

$$
0 \leq \operatorname{area}^{\prime \prime} \leq \int_{Y_{\min }} K\left(Y_{\min }\right) d y+\int_{\partial Y_{\min }} \operatorname{curv}\left(\partial Y_{\min }\right) d s,
$$

becomes

$$
0 \leq \operatorname{area}^{\prime \prime} \leq \int_{Y_{\text {min }}} K\left(Y_{\text {min }}\right) d y+\int_{\partial Y_{\text {min }}} \operatorname{curv}\left(\partial Y_{\text {min }}\right) d s-\kappa \cdot \operatorname{area}\left(Y_{\text {min }}\right)
$$

and

$$
\sum_{i}\left(\pi-\alpha_{i}\right)+\kappa \cdot \operatorname{area}\left(Y_{\min }\right) \leq 2 \pi \chi\left(Y_{\min }\right)
$$

Notice that this is sharp (i.e. turns into an equality) for $P$ being the product of a $k$-gonal surface $Q_{\kappa}^{2}$ of constant curvature $\kappa$ by a line segment.

Also observe that the area of $Y_{\min }$ is bounded from below by an $A$, (that is of use for $\kappa>0$ ) if $P$ admits a 1-Lipschitz map $f$ onto a disk $D$ of area $A$, such that $f$ sends the side-boundary of $P$ on $\partial D$ with the top and the bottom of $P$ being sent to $D$ with degree 1 .

On the other hand, area $\left(Y_{\min }\right) \leq A$ (that may be used for $\kappa<0$ ) if the distance $d$ between the top and bottom in $P$ is related to the volume of $P$ by $\operatorname{vol}(P) / d \leq A$.

Semi-Integral Inequality. If $\kappa>0$, then the inequality $\operatorname{scal}(P) \geq \kappa$ can be significantly relaxed by requiring that the integral $\int_{Y} \operatorname{scal}(P) d y$ is bounded from below by $2 \kappa \cdot \operatorname{area}\left(Q_{\kappa}^{2}\right)$ for the above $k$-gon $Q_{\kappa}^{2}$ and all surfaces $Y \subset P$ separating the top from the bottom.

The resulting inequality (compare [42] is most informative if $\operatorname{scal}(P) \geq 0$ and it can be also meaningfully used if $\operatorname{scal}(P) \geq \kappa_{-}$for some $\kappa_{-}<0$ if one also has an upper bound on $\operatorname{area}\left(Y_{\min }\right)$. For example, this shows the following.

Given an Riemannian metric on $P$, it can not modified with an uncontrolled enlargement of its scalar curvature along $N$ line segments joining the top to the bottom of $P$, e.g. by inserting $N$ tubes of fixed thickness, say $\delta$ independent of $N$, with scalar curvatures $\geq \varepsilon=\frac{100}{N \delta^{2}}$ and large $N$ without generating a proportional amount of negative scalar curvature.

On Non-zero bounds on the Mean Curvatures of the Faces of P. Besides allowing $\kappa \neq 0$ one may similarly allow non-zero lower bounds on the mean curvature by some numbers of the side faces of $P$ by some $m_{i}, i=1,2, \ldots, k$.

If $m_{i}>0$, this may be used together with a lower bound on the "widths" of these faces, but it is less clear what kind of upper bound on the size of these faces (and/or on all of $P$ ) may serve along with some $m_{i}<0$.

$K$-Area and Semi-integral Inequalities for Closed and for Cornered $n$-Manifolds. Let $X$ be a closed oriented $n$-dimensional Riemannian manifold, 
where the fundamental cohomology class $[X]^{n} \in H^{n}(X ; \mathbb{Z})$ equals the - -product of 2-dimensional classes and a class $h^{k} \in H^{k}(X ; \mathbb{Z})$ coming from $H^{k}(\Gamma ; \mathbb{Z})$ under the classifying map $X \rightarrow K(\Gamma ; 1)$.

An instance of such an $X$ is a Cartesian product of complex projective spaces, e.g. of 2 -spheres, and of a closed $k$-manifold $Z$ of non-positive sectional curvature, e.g. $Z=\mathbb{T}^{k}$.

Denote by $\operatorname{sc} . \operatorname{ar}(X)$ the infimum of the numbers $A$, such that the $N$-th multiples of all non-zero integer 2-dimensional homology classes in $X$ are representable by surfaces $Y$ in $X$ (possibly, with self intersections), such that

$$
\int_{Y} \operatorname{scal}(X) d y \leq N \cdot A \text { for all } N=1,2,3, \ldots .
$$

Notice that this definition makes sense only if $\operatorname{scal}(X) \geq 0$; otherwise, $\operatorname{sc} . \operatorname{ar}(X)=-\infty$.

Let the universal covering $\tilde{X}$ of the manifold $X$ be spin, e.g. homeomorphic to a product of 2 -spheres, or more generally, of complex projective spaces $\mathbb{C} P^{l}$ for odd $l$, and of $\mathbb{R}^{k}$.

Also assume that the class $h^{k}$ has infinite $K$-area in the sense of [19], e.g. this class comes from (a cohomology class of) a complete manifold $Z^{\prime}$ of non-positive curvature by a map $X \rightarrow Z^{\prime}$. $X$.

Then sc.ar $(X) \leq$ const $_{\text {top }}$ where const $t_{\text {top }}<\infty$ depends on the topology of

Proof. By the Whitney-Hahn-Banach (duality) theorem, the fundamental class of $X$ is representable by a product of smooth closed 2 -forms $\omega_{i}$ with their sup-norms bounded by

$$
\left\|\omega_{i}\right\|_{\text {sup }} \leq \kappa+\varepsilon \text { for } \kappa=(\operatorname{scar}(X))^{-1} \text { and all } \varepsilon>0 .
$$

Since all $\omega_{i}$ are representable as curvature forms of complex line bundles over $X$, theorem $5 \frac{1}{4}$ from [19] applies and the proof follows.

Remark/Question. the Riemannian products of 2-spheres by the torus, are probably extremal for this inequality, i.e. $\operatorname{sc} . \operatorname{ar}(X)=$ const $_{\text {top }}$ for these $X$.

This seems to follow from (a suitable form of) the area extremality of the product of 2-spheres with arbitrary metrics of positive curvatures with the flat torus $\mathbb{T}^{k}$, see [41], [32], [33], [12], but I did not hat it carefully.

More general products of complex projective spaces with Kähler metrics, $\left(\times_{i} \mathbb{C} P^{l_{i}}\right) \times \mathbb{T}^{k}$ are also extremal [13] and this probably, extends to singular metrics with curvatures concentrated along divisors.

Potential Corollary. Let $P$ be an $n$-dimensional mean curvature convex cornered spin manifold that is combinatorially equivalent to a Cartesian product $P_{0}$ of reflection domains in the spheres $S_{i}^{2}$ and in $\mathbb{R}^{k}$.

Observe that such a $P$ serves as a reflection domains in a closed manifold $\hat{X}$ that admits a map with degree one onto a product of 2 -spheres $S_{i}^{2}$ and the torus $\mathbb{T}^{k}$.

Let $\operatorname{scal}(P)=0$, e.g. $P$ is isometric to a cornered domain in $\mathbb{R}^{n}$.

Then the above implies a certain lower bound on the dihedral angles $\alpha_{j}$ of $P$ as follows.

Assume, to simplify the notation, that the above reflection (Cartesian product) domain $P_{0}$ has its all dihedral angles $\pi / 2$. Thus all all spherical reflection domains $P_{i} \subset S_{i}^{2}$ in this case are spherical triangles with $90^{\circ}$ angles. 
Let us endow the above closed manifold $\hat{X} \supset P$ (obtained by reflecting $P$ ) with the singular Riemannian metric $\hat{g}$ that extends that on $P$ and observe that the essential contribution to (non-negative!) scalar curvature of this metric comes from the edges, i.e. codimension 2 faces of $P$.

Namely, the contribution of such an edge with the dihedral angle $\alpha_{j}$ to a surface $Y$ transversally intersecting the "descendent" of this edge in $X$ equals $2 \pi-4 \alpha_{j}$.

Thus, assuming the truth of the above extremality statement for of $\times S_{i}^{2} \times \mathbb{T}^{k}$,

the sums $\Sigma_{3}$ of all triples of dihedral angles in $P$ corresponding to the triples of vertices of the reflection triangles in the spheres $S_{i}^{2}$ are bounded from below by $\Sigma_{3} \geq \pi$.

Notice that we do not have to take $N$-multiples of our 2-dimensional homology classes in this case.

Also observe that if $n=3$, then the above reduces to a special case of the Gauss-Bonnet prism inequality, namely for "triangular" prisms $P^{3}=\Delta \times[0,1]$.

Remarks on Pure Edge Singularities. The $K$-area inequalities and the related extremality/rigidity results for closed for Riemannian manifolds $(X, g)$ can be expressed in terms of the size/shape of this $X$ with the metric $\operatorname{scal}(g) \cdot g$, where, observe this metric is invariant under scaling of $g$. (Compare [41, [32], [33, 12.)

Namely, the suitable for the present purpose area extremality of an $X$ says that if another manifold, say $\left(X^{\prime}, g^{\prime}\right)$ admits a map $f: X \rightarrow X^{\prime}$ of positive degree than this $f$ can not be strictly area decreasing with respect to the metrics $\operatorname{scal}(g) \cdot g$ and $\operatorname{scal}\left(g^{\prime}\right) \cdot g^{\prime}$.

The case that is relevant in the present context is that of singular metrics $g$ with the singularity, say $\Sigma \subset X$ being of codimension 2 and with the main contribution to the scalar curvature being supported on $\Sigma$ transversally to $\Sigma$.

One may think of this $\Sigma \subset X$ as a "divisor" in $X$ (divisors in complex manifolds $X$ provide a pool of interesting examples) and the $K$-area formulas apply to the Dirac operator twisted with the line bundle associated to this "divisor" or to Whitney sums of such bundles.

This is what we have actually done for the above "prisms" and it would be interesting to look more systematically on more general such $(X, \Sigma)$.

Question. Can the $K$-area be used for bounding from below some "combinatorial sizes/shapes" of mean curvature convex polyhedra $P$ in $\mathbb{R}^{n}$, say for $n=3$, with all their dihedral angles bounded by $\pi-\varepsilon$ for a given $\varepsilon>0$ ?

Hyperbolic Prism Inequality. The $3 D$ Gauss-Bonnet prism inequality was "modeled" on the prisms in the product spaces $V_{\kappa}^{2} \times \mathbb{R}$ for surfaces $V_{\kappa}^{2}$ of constant curvature $\kappa$.

Now let us look at prisms $P_{\text {horo }}$ in the hyperbolic space $H^{3}$ of curvature -1 where the top and the bottom are contained in two parallel horospheres. Observe that the side faces of $P_{\text {horo }}$ are totally geodesic, and the mean curvatures of the top and the bottom equal +1 and -1 respectively.

Now, let $P$ be a normal prism with $\operatorname{scal}(P) \geq-6=\operatorname{scal}\left(H^{3}\right)$ where the side faces are mean curvature convex while the mean curvatures of the top and of the bottom are bounded from below by +1 and -1 .

The above argument, applied to constant mean curvature bubbles $Y_{b b l} \subset P$ 
(compare $\S 5 \frac{5}{6}$ in $[19]$ ), implies that

$$
\sum_{i=1, \ldots, k}\left(\pi-\alpha_{i}\right) \leq 2 \pi
$$

where the equality holds if and only if $P$ is isometric to some $P_{\text {horo }}$ in the hyperbolic space $H^{3}$.

On Spherical Prisms. Let is indicate a similar extremality/rigidity property of spherical rather than horospherical prisms that applies to 3 -manifolds $V_{\kappa}^{3}$ of constant curvature $\kappa$ for all $-\infty<\kappa<+\infty$.

Let $P_{s p h} \subset V_{\kappa}^{3}$ be a normal prism where the top and the bottom lie in two concentric spheres in $V_{\kappa}^{3}$ (or two concentric umbilical surfaces if $\kappa<0$ ) and where the side faces of $P_{s p h}$ are totally geodesic. Denote by $Y_{s p h}(d) \subset P_{s p h}$ the $d$-level of the distance function to the (concave) bottom of $P_{s p h}$ and let $m(d)$ denotes the mean curvature of $Y_{s p h}(d)$.

Observe that the scalar curvature of $Y_{s p h}(d)$ equals $\operatorname{scal}\left(V_{\kappa}^{3}\right)+\frac{3}{4} m(d)^{2}$, compare $\S 5 \frac{5}{6}$ in 19 , and, thus, the number

$$
S={ }_{\text {def }} \operatorname{area}\left(Y_{\text {sph }}\right)(d) \cdot\left(\operatorname{scal}\left(V_{\kappa}^{3}\right)+\frac{3}{4} m(d)^{2}\right)
$$

is independent of $d$ and where, clearly, $S \geq 0$ for all $\kappa$.

Let $a_{i}, i=1, \ldots, k$, denote the dihedral angles between the side faces of $P_{s p h}$.

Let $P$ be a normal Riemannian prism combinatorially equivalent to $P_{s p h}$ (with not necessarily $\operatorname{scal}(P) \geq \operatorname{scal}\left(V_{\kappa}^{3}\right)$ ) where the side faces of $P$ are mean curvature convex while the mean curvatures of the top and the bottom are bounded from below by those of $P_{s p h}$. Thus top of $P$ is mean convex while the bottom may be concave.

Let $\delta=\delta(p)$ denotes the distance from $p$ to the bottom of $P$.

Let the distance between the top and bottom in $P$ is (non-strictly) greater than that in $P_{s p h}$ and let

$$
\int_{Y}\left(\operatorname{scal}(P)+\frac{3}{4} m(\delta)^{2}\right) d y \geq S
$$

for all surfaces $Y \in P$ that separate the top from the bottom.

Then the upper bounds $\alpha_{i}$ on the dihedral angles between the side faces of $P$ satisfy

$$
\sum_{i} \alpha_{i} \geq \sum_{i} a_{i}
$$

The above spherical prism inequality can be applied to particular small domains $P$ in 3-manifolds $X$ delivered by an argument similar for proving (ם) from 5.9 , where it shows that

If a $C^{2}$-metric $g$ on a 3-manifold equals a $C^{0}$-limit of $C^{2}$-metrics $g_{i}$ with $\operatorname{scal}\left(g_{i}\right) \geq \kappa$ for a given $-\infty<\kappa<+\infty$, then $\operatorname{scal}(g) \geq \kappa$.

In fact, one needs for this purpose only polyhedral domains of depth 2 , i.e. without corners, namely, those combinatorially equivalent to round 3-cylinders, where the relevant bubbles, that separate top from the bottom, are $C^{2}$-smooth at the boundary. 
Question. Can one prove all of the above "prism inequalities" by a purely Dirac-type argument (e.g. elaborating on that in [40] and/or in [10] and where [36] may be relevant) with no localization to minimal hypersurfaces?

More on Pure Edges. The study of the above $P$ can be reduced by (multi)doubling to the "pure edge" picture (see 1.1., 2.3).

Then one observes that the semi-integral inequalities are stable under smoothing of the edges since these smoothings that do not significantly change the integrals of the scalar curvatures over the relevant surfaces; thus, one can fully avoid the "singularities at the corners" problem.

It seems that the geometry of manifolds $X$ of all dimensions with scal $\geq 0$ (and, possibly, with $s c a l \geq \kappa$ ) is governed by integrals of $\operatorname{scal}(X)$ over surfaces in $X$, or, rather by infima of such integrals over surfaces $A$ with (small?) boundaries $B$ and with $A$ being "not-too-far" from $B=\partial A$.

However, this is insufficient, for instance, for extending the (dual) Kirszbraun theorem to mean curvature convex simplices $P=\Delta_{\text {curv }}^{3}$ with $\operatorname{scal}(P) \geq 0$ that is needed for proving the dihedral extremality of the ordinary simplices $\Delta^{3} \subset \mathbb{R}^{3}$ (see section 1.6).

But, possibly, this may be achieved with $\mu$-bubbles $Y \subset P, \partial Y \subset \partial P$, that are obtained by inward deformations of the surface $Y_{0} \subset \partial P=\partial \Delta_{\text {curv }}^{3}$, for $Y_{0}$ being $\partial \Delta_{\text {curv }}^{3}$ minus four small discs around four vertices of $\Delta_{\text {curv }}^{3}$, and where $\mu$ are suitable measures supported on $\partial \Delta_{\text {curv }}^{3}$.

Such $Y$ do not have to be normal to the edges that may increase the integral of the scalar curvatures of (regularized meteoric on) $\Delta^{3}$ over $Y$.

More on n-Dimensional "Prisms", etc. Application of minimal hypersurfaces (see [46], 25] depends on their regularity. This limits one to $n \leq 7$, where the case $n=8$ follows from the removal of isolated singularities [44] with the general case, probably, amenable to Lohkamp's "around singularities" method. (Removal and/or "going around" singularities is good for proving extremality of cornered domains but it is poorly adjusted for rigidity.)

But even disregarding the singularity problems, (including that at the corners) one needs to adjust the warping from [25] to the above Gauss-Bonnet argument.

On the other hand, as we saw above, the Dirac operator $/ K$-area method applies in some cases.

Furthermore, let for example, $Y_{\text {horo }}^{n-1}$ be a Euclidean reflection domain in a horosphere in the hyperbolic $n$-space, then the study of the corresponding prisms reduces to that of manifolds homeomorphic to $\mathbb{T}^{n-1} \times \mathbb{R}$, where the corresponding result is proven in 19 for $n \leq 7$. This sharpens/quantifies Min-Oo's rigidity theorem 40, 41, for hyperbolic $n$-spaces in the case $n \leq 7$ (with 44, one gets extremality but not rigidity for $n=8$ ) but leaves $n \geq 9$ open. (Possibly, Lohkamp's method applies here.)

What are, in general, extremal polyhedral objects in symmetric spaces $X$ of non-constant curvatures?

If $X$ is a Cartesian product of constant curvature spaces, then natural candidates are Cartesian products of poly-bubble-hedra but it is unclear waht happens in general. On the other hand, there are several rigidity (and non-rigidity) results for compact and complete manifolds without boundary [40, [41] 34] [32] 7 . 
Model Problem. Is there a single theory of manifolds $X$ with $\operatorname{scal}(X) \geq \kappa$ or there may be several theories associated with different (homogeneous? symmetric?) model spaces that serve as extrema for geometric/topological inequalities in spaces with $s c a l \geq \kappa$ ?

Rigidity and Stability. All(?) geometric/topological inequalities, in particular, those concerning (smooth as well as cornered) manifolds $X$ with $\operatorname{scal}(X) \geq$ $\kappa$, whenever these are sharp, are accompanied by rigidity problems where one asks for a description of $X$ where such inequalities become equalities.

But even when such rigidity of an $X$ is known, e.g. for $X=\mathbb{T}^{n}$ with flat metric, it is not quite clear in what sense such an $X$ is stable. For example, one can (almost) unrestrictedly blow huge "bubbles" with positive scalar curvature "grounded" in codimension $k$ subsets in a Riemannian manifold $X$ for $k \geq 3$. But the above semi-integral inequalities indicate that this is impossible with $k \geq 2$.

This, probably, can be interpreted as follows:

there is a particular "Sobolev type weak metric" dist wea in the space of $n$ manifolds $X$, such that, for example, tori $X$ with $\operatorname{scal}(X) \geq-\varepsilon$, when properly normalized, (sub)converge to flat tori, $\operatorname{dist}_{\text {wea }}\left(X, \mathbb{T}_{\text {flat }}^{n}\right) \rightarrow 0$ for $\varepsilon \rightarrow 0$, but these $X$ may, in general, diverge in stronger metrics.

(A preliminary step toward construction of such a metric can be seen in [48.)

\subsection{Spaces or Objects?}

The Dirac operator $D$ and minimal hypersurfaces seem to belong to different wolds but they unexpectedly meet in spaces with lower bounds on their scalar curvatures.

Is there a deeper link between "Dirac" and "minimal".

It should be noted that the constrains on the geometry of manifolds $X$ with $\operatorname{scal} \geq 0$ (and $\operatorname{scal}(X) \geq \kappa$, in general), and the issuing constrains on the topology of $X$, that obtained with the Dirac operator are significantly different from those obtained with minimal hypersurfces, even for spin manifolds of dimension $\leq 7$ where both methods apply.

An essential drawback of a direct application of Dirac operators is the requirement of completeness of $X$, while the use of minimal surfaces in dimensions $n \geq 4$ delivers upper distance bounds rather than area bounds as the Dirac operators do. (Particularly fine results can be obtained for $\operatorname{dim}(X)=4$ with a use of the Seiberg-Witten equation but this is fully beyond the scope of the present paper.)

Can one unify the two methods and, thus, obtains more precise results?

The key to the application of Dirac to scalar curvature is the Lichnerowicz formula $D^{2}=\nabla^{2}+$ scal $/ 4$ where $\nabla^{2}$ is more positive than an ordinary Laplace operator, while the application of minimal (hyper)surfaces depends on the second variation formula in the Schoen-Yau form that makes it similar to Lichnerowicz formula.

Are there further formulas of this kind mediating between these two?

The next step in approaching the problem is to change our perspective on manifolds in the spirit of ideas of Fedia Bogomolov and Maxim Kontsevich. 
Fedia suggested looking at stable vector bundles with zero $c_{1}$ on an algebraic variety $X$ as at "coherent families" of flat bundles over the curves $C \subset X$, while Maxim's idea was to regard Riemannian manifolds as (special cases of) functors from the "category of graphs" to the category of measure spaces: the value of such a functor $F$ on a graph $G$ with given edge lengths is the space of maps $G \rightarrow X$ with the Wiener measure on it.

If we want to see the Dirac operator in Maxim's picture, we have to consider graphs $G$ along with flat $O(n)$-bundles $V, n=\operatorname{dim} X$, over them and with an embedding of the "tangent spaces" of the graphs at all points $g \in G$ into $V_{g}$ (that is most informative at the vertices $g \in G$ of high valency). Then one needs to define an appropriate structures, including measures, on the the spaces of maps $(G, V) \rightarrow(X, T(X))$ for the tangent bundle $T(X)$.

The flat structures in $V$ encode the parallel transport in $X$ that allows one to speak of "Dirac" as well as of other geometric differential operators. On the other hand, a measure on the space of maps $G \rightarrow X$ allows an integration of the numbers of intersections of these graphs with hypersurfaces $Y \in X$, thus keeping track of the volumes of these $X$.

Besides all this, one can associate with every cycle in $G$ the range of values of the areas of surfaces $A$ fillings this cycles in $X$ along with the integrals $\int_{A} \operatorname{scal}(X) d a$, where, possibly, the values of the genera and Euler characteristics of $A$ may be also relevant.

Possibly, the spirit of semi-integral inequalities suggests that we may actually forfeit graphs and think of $X$ as a contravariant functor from a suitable category of surfaces to some set category.

Final Questions. Can one make a mathematical theory along these lines with manifolds being replaced by objects of a more abstract and more flexible category of functors from a category of "extended objects" to sets?

Can one, thus, extend basic results on positive scalar curvature to singular and/or infinite dimensional spaces?

\section{Bibliography.}

\section{References}

[1] W. K. Allard, On the first variation of a varifold, Annals of Math 95 (1972), 417-491.

[2] Sebastiao Almeida, Minimal Hypersurfaces of a Positive Scalar Curvature. Math. Z. 190, 73-82 (1985) Mathematische Zeitschrift

[3] F. Almgen, Existence and regularity almost everywhere of solutions to elliptic variational problems among surfaces of varying topological type and singularity structure, Annals of Mathematics 87 (1968), 321-391.

[4] F.Almgren, Optimal isoperimetric inequalities, Indiana University Mathematics Journal 35 (1986), no. 3, 451-547.

[5] Andreas Bernig, Scalar curvature of definable CAT-spaces, Adv. Geom. 3 (2003), 23?43 
[6] Brakke, Kenneth, A. Minimal surfaces, corners, and wires. J. Geom. Anal. 2 (1992), no. 1, 11-36.

[7] Brendle, F. C. Marques, A. Neves, Deformations of the hemisphere that increase scalar curvature. arXiv:1004.3088v1 [math.DG] 19 Apr 2010.

[8] Burago, Ju.D.; Toponogov,V. A., Three-dimensional Riemannian spaces with curvature bounded from above. (Russian) Mat. Zametki 13 (1973), 881-887.

[9] J Cheeger, TH Colding, On the structure of spaces with Ricci curvature bounded below. I Journal of Differential Geometry, Volume 46, Number 3 (1997), 406-480.

[10] Hèléne Davaux, An Optimal Inequality Between Scalar Curvature and Spectrum of the Laplacian Mathematische Annalen, volume 327 issue 2 (30 September 2003), pages 271-292

[11] Michael Eichmair, Pengzi Miao, Xiaodong Wang, Extension of a theorem of Shi and Tam arXiv:0911.0377 [math.DG].

[12] S. Goette and U. Semmelmann. Scalar curvature estimates for compact symmetric spaces. Differential Geom. Appl., 16(1):65?78, 2002.

[13] S. Goette and U. Semmelmann, $\operatorname{Spin}^{c}$ structures and scalar curvature estimates, Annals of Global Analysis and Geometry 20 (4): 301-324 , 2001

[14] James D. E. Grant, Nathalie Tassotti A positive mass theorem for lowregularity metrics. arXiv:math-ph/0212025

[15] M. Gromov, Filling Riemannian manifolds, GDG, 18 (1983) 1-147.

[16] M. Gromov, Sign and geometric meaning of curvature, Rend. Semin. Mat. Fis. Milano 61 (1991), 9 - 123.

[17] M. Gromov, Foliated Plateau problem, part I. GAFA 1, 14-79 (1991).

[18] M. Gromov, Metric invariants of Kähler manifolds, Differential geometry and topology (Alghero, 1992), 90 -116, World Sci. NJ, 1993.

[19] M. Gromov, Positive curvature, macroscopic dimension, spectral gaps and higher signatures. in Functional Analysis on the Eve of the 21st Century: In Honor of the 80th Birthday of I.M. Gelfand, Progress in Mathematics, Volume 131 of Progress in Mathematics Series, 1995.

[20] M. Gromov, Singularities, Expanders and Topology of Maps. Part 2: from Combinatorics to Topology Via Algebraic Isoperimetry. Geom. func. anal., 20 (2010), 416-526.

[21] M. Gromov, Hilbert volume in metric spaces. Part 1, Central European Journal of Mathematics (April 2012), 10 (2), pg. 371-400

[22] M. Gromov. Plateau-Stein manifolds. http://www.ihes.fr/ gromov/

[23] M. Gromov and H. B. Lawson, Jr., Spin and scalar curvature in the presence of a fundamental group, Ann. of Math. Ill (1980), 209-230. 
[24] M. Gromov and H. B. Lawson, Jr., The classification of simply connected manifolds of positive scalar curvature, Ann. of Math. 111 (1980), 423-434.

[25] M. Gromov and H. B. Lawson, Jr., Positive scalar curvature and the Dirac operator on complete Riemannian manifolds, Publ. Math. I.H.E.S., 58 (1983), 83-196 .

[26] Grüter, Michael, Optimal regularity for codimension one minimal surfaces with a free boundary. Manuscript Math. 58 (1987), 295-343.

[27] Grüter, Michael, Jost, Jürgen, Allard type regularity results for varifolds with free boundaries. Annali della Scuola Normale Superiore di Pisa, Classe di Scienze 4e srie, tome 13, no 1. ( 1986)

[28] J. Kazdan and F. Warner, Prescribing curvatures, Proc. of Symp. in Pure Math. 27 (1975), 309-319

[29] Kinderlehrer, D., Nirenberg, L., Regularity in free boundary problems. Ann. Scuola Norm. Sup. Pisa Cl. Sci. (4) 4 (1977), no. 2, 373-391.

[30] G. La Nave, Macroscopic dimension and fundamental group of manifolds with positive isotropic curvature, Preprint Summer 2012 http://www.math.uiuc.edu/lanave/macrodimisotrop3.pdf.

[31] Dan A. Lee, A positive mass theorem for Lipschitz metrics with small singular sets. arXiv:1110.6485.

[32] Mario Listing, Scalar Curvature on Compact Symmetric Spaces arXiv:1007.1832 [math.DG]

[33] Mario Listing, Scalar Curvature and vector bundles, arXiv:1202.4325 [math.DG]

[34] . M. Llarull, Scalar curvature estimates for $(\mathrm{n}+4 \mathrm{k})$-dimensional manifolds. Diff. Geometry and its Applications, Volume 6 (1996) 321-326.

[35] Lohkamp J., Positive scalar curvature in dimension! 8 C. R. Math. Acad. Sci. Paris 343 (2006), no. 9, 585-588

[36] Lusztig, George, Cohomology of classifying spaces and Hermitian representations. Represent. Theory 1 (1997), 31-36.

[37] Donovan McFeron and Gbor Szkelyhidi, On the positive mass theorem for manifolds with corners. Preprint arXiv:1104.2258

[38] M. Micallef and J. D. Moore, Minimal two-spheres and the topology of manifolds with positive curvature on totally isotropic two-planes, Annals of Math. 127 (1988), 199-227.

[39] P. Miao. Positive mass theorem on manifolds admitting corners along a hypersurface. Adv. Theor. Math. Phys., 6(6):1163-1182, 2002.

[40] M. Min-Oo, Scalar curvature rigidity of asymptotically hyperbolic spin manifolds. Math. Ann. 285, 527- 539 (1989) 
[41] M. Min-Oo, Dirac Operator in Geometry and Physics in Global Riemannian Geometry: Curvature and Topology; Advanced courses in Mathematics, CRM Barcelona, Birkhauser (2003).

[42] Anton Petrunin, An upper bound for curvature integral. St. Petersburg Math. J. 20 (2009), 255-265

[43] E. R. Reifenberg, Solution of the Plateau problem for m-dimensional surfaces of varying topological type, Acta Math. 104 (1962) 1-92.

[44] Smale, N, Generic regularity of homologically area minimizing hypersurfaces in eight dimensional manifolds, Commun. Anal. Geom. 1, (1993) 217228

[45] R. Schoen and S. T. Yau, Existence of incompressible minimal surfaces and the topology of three dimensional manifolds of non-negative scalar curvature, Ann. of Math. 110 (1979), 127-142.

[46] R. Schoen and S. T. Yau, On the structure of manifolds with positive scalar curvature, Manuscripta Math. 28 (1979), 159-183.

[47] Leon Simon, Regularity of capillary surfaces over domains with corners. Leon Simon, Pacific J. Math. Volume 88, Number 2 (1980), 363-377.

[48] Christina Sormani and Stefan Wenger, The Intrinsic Flat Distance between Riemannian Manifolds and Integral Current Spaces, Journal of Differential Geometry, Vol. 87 (2011) 117-199.

[49] Stefan Wenger, A short proof of Gromov's filling inequality Proc. Amer. Math. Soc. 136 (2008), 2937-2941. 